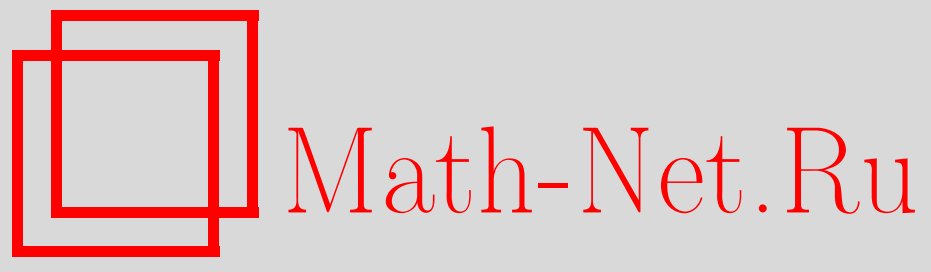

Вик. С. Куликов, Кривые Гурвица, УМН, 2007, том 62, выпуск $6,3-86$

DOI: https://doi.org/10.4213/rm8530

Использование Общероссийского математического портала Math-Net.Ru подразумевает, что вы прочитали и согласны с пользовательским соглашением http: //www . mathnet.ru/rus/agreement

Параметры загрузки:

IP: 54.89 .56 .158

26 апреля 2023 г., 16:20:25

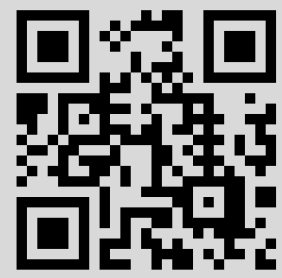




\section{Кривые Гурвица}

\section{Вик. С. Куликов}

Данная статья является обзором полученных в последнее время результатов о кривых Гурвица, о их брэйд-монодромных инвариантах и их приложениях к проблемам $H$-изотопности и регулярной гомотопности кривых Гурвица.

Вторая часть обзора посвящена обсуждению возможности применения брэйд-монодромных инвариантов кривых ветвления общих накрытий проективной плоскости в качестве инвариантов, различающих связные компоненты пространства модулей алгебраических поверхностей (в алгебраическом случае) и различающих симплектические структуры на четырехмерных многообразиях (в симплектическом случае).

Библиография: 64 названия.

\section{СОДЕРЖАНИЕ}

Введение

$\S 1$. Кривые Гурвица на поверхностях Хирцебруха, псевдоголоморфные кривые и симплектические поверхности ................... 12

1.1. Определение кривых Гурвица........................ 12

1.2. Симплектические и почти комплексные структуры .......... 14

1.3. Симплектические кривые Гурвица...................... 16

$\S 2$ Полугруппы разложений на множители . $\ldots \ldots \ldots \ldots \ldots \ldots \ldots \ldots \ldots . \ldots \ldots$

2.1. Полугруппы разложений на множители . . . . . . . . . . . 17

2.2. Полугруппы разложений на множители с частичным сокращением . . . . . . . . . . . . . . . . . . . . . . . . . . . . . . . . 19

2.3. Эквивалентность Гурвица............................ 19

2.4. Регенерация разложений на множители ................. 20

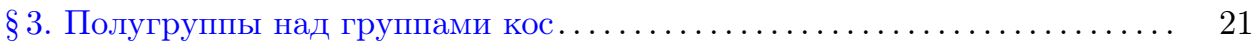

3.1. Реализации группы кос ............................. 21

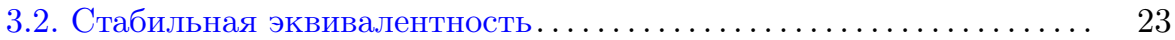

3.3. Формула разложения на множители полного поворота с удвоен-

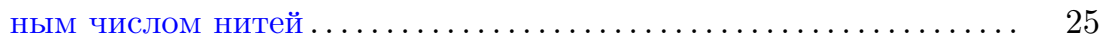

3.4. Группы, ассоциированные с элементами $s \in S_{\mathrm{Br}_{m}} \ldots \ldots \ldots \ldots .26$

Данная работа частично поддержана грантами: РФФИ 05-01-00455, НШ-489.2003.1, NWO-RFBR 047.011.2004.026 (РФФИ 05-02-89000-HBО_a), РФФИ 06-01-72017-MNTI_a, РФФИ 07-01-92211-НЦНИЛ_а, INTAS (05-7805) и CRDF RUM1-2692-MO-05.

(C) Вик. С. Куликов, 2007 


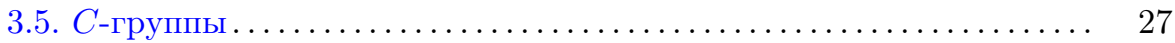

3.6. Гурвицевские $C$-группы .............................. 30

3.7. Модули Александера и многочлены Александера $C$-групп ...... 30

3.8. Вычисление модулей Александера $C$-групп ............... 31

3.9. Модули Александера неприводимых $C$-групп............... 33

3.10. Свойства нётеровых $(t-1)$-обратимых $\Lambda$-модулей.......... 34

$\S 4$. Разложение на множители брэйд-монодромии кривой Гурвица . . . . . 36

4.1. Локальная брэйд-монодромия над точкой (случай одного ростка) 36

4.2. Локальная брэйд-монодромия над точкой (случай нескольких

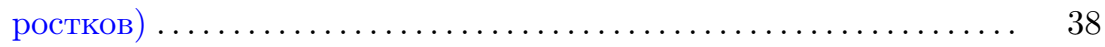

4.3. Разложение на множители брэйд-монодромии над диском . . . . . 40

4.4. Разложение на множители брэйд-монодромии кривой Гурвица . 44

4.5. Брэйд-монодромное разложение на множители псевдоголоморфной кривой в $\mathbb{C P}^{2} \ldots \ldots \ldots \ldots \ldots \ldots \ldots \ldots \ldots \ldots \ldots \ldots \ldots \ldots \ldots \ldots \ldots \ldots \ldots \ldots \ldots \ldots \ldots$

4.6. Гомотопический тип дополнения к аффинной кривой Гурвица. 46

$\S 5$. Деформации и $H$-изотопии кривых Гурвица ................. 46

5.1. Эквисингулярные деформации кривых Гурвица............ 46

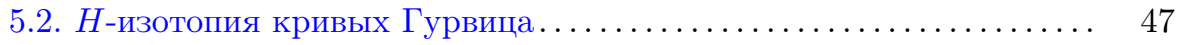

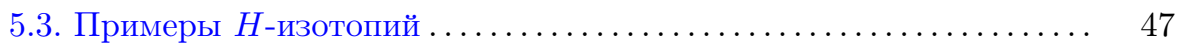

5.4. Представление кривых Гурвица $\mathbb{C P}^{2}$ в виде нулей сечений ли-

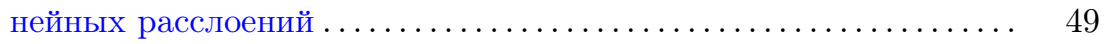

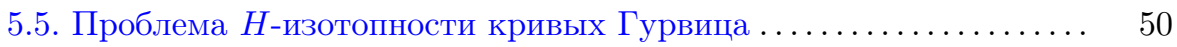

5.6. Критерий $H$-изотопности каспидальных кривых Гурвица...... 51

5.7. H-изотопность кривых Гурвица с особыми точками нерасщеп-

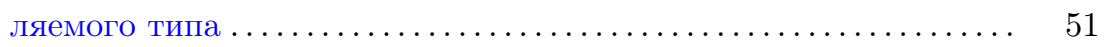

5.8. Изотопность псевдоголоморфных кривых в $\mathbb{C P}^{2} \ldots \ldots \ldots \ldots \ldots . \ldots 3$

5.9. Сравнение множеств алгебраических и псевдоголоморфных кри-

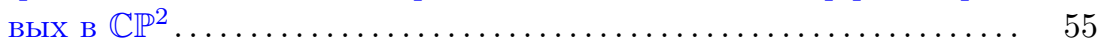

5.10. Полугруппа $\operatorname{Hur}_{m}$ над группой кос $\mathrm{Br}_{m} \ldots \ldots \ldots \ldots \ldots \ldots \ldots \ldots . \ldots . \ldots . \ldots . \ldots . \ldots$

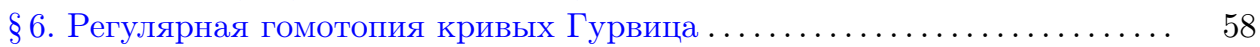

6.1. Рождение и уничтожение пары ноудов ................ 58

6.2. Критерий регулярной гомотопности каспидальных кривых Гур-

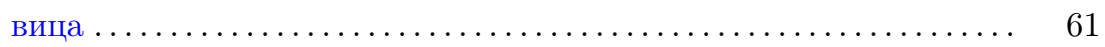

$\S 7$. Фундаментальные группы дополнений к кривым Гурвица......... 62

7.1. Теоремы Зариского-ван Кампена....................... 62

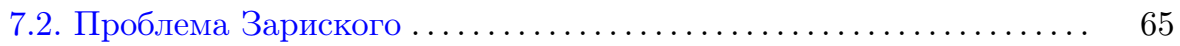

$\S 8$. Алгебраические разветвленные накрытия проективной плоскости ... 66

8.1. Монодромия накрытия............................. 66

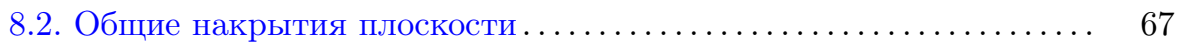

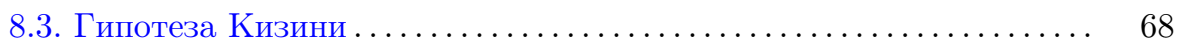

8.4. Деформации комплексных структур на проективных алгебраических поверхностях......................... 69

$\S 9$. Конечнолистные симплектические накрытия проективной плоскости . 72

9.1. Построение конечнолистных накрытий проективной плоскости, разветвленных вдоль кривых Гурвица ............... 72

9.2. Общие симплектические накрытия проективной плоскости . . . . 74 
$\S 10$. Циклические накрытия проективной плоскости . . . . . . . . . . . .

10.1. Вложение циклических накрытий плоскости в проективные комплексные трехмерные рациональные многообразия ........

10.2. Модули Александера и многочлены Александера кривых Гур-

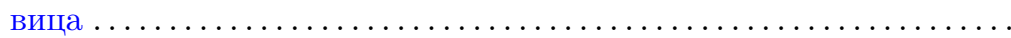

10.3. Вычисление группы первых гомологий циклического накрытия плоскости . . . . . . . . . . . . . . . . . . . . . . . . .

\section{Введение}

Начиная с основополагающих работ Б. Римана, одним из основных методов исследования алгебраических и комплексно-аналитических многообразий является представление этих многообразий в виде конечнолистных разветвленных накрытий проективных пространств той же размерности (или областей в проективных пространствах в комплексно-аналитическом случае). Так, риманова поверхность - это конечное накрытие области в $\mathbb{C P}^{1}$, разветвленное в конечном числе точек. В частности, каждая проективная алгебраическая кривая $C$ может быть представлена в виде такого конечного накрытия $f: C \rightarrow \mathbb{C P}^{1}$, разветвленного в конечном числе точек. Если $q_{1}, \ldots, q_{n} \in \mathbb{C P}^{1}-$ точки, над которыми разветвлено накрытие $f$, и $m$ - его степень, то каждая петля $\gamma \subset \mathbb{C P}^{1} \backslash\left\{q_{1}, \ldots, q_{n}\right\}$ с началом в некоторой фиксированной точке $q_{0}$ может быть поднята на $C$ с помощью $f$ и мы получим $m$ путей, начинающихся и заканчивающихся в точках, лежащих в $f^{-1}\left(q_{0}\right)$. Этот подъем определяет гомоморфизм (монодромию накрытия) $\mu: \pi_{1}\left(\mathbb{C P} \backslash\left\{q_{1}, \ldots, q_{n}\right\}, q_{0}\right) \rightarrow \mathscr{S}_{m}$ в симметрическую группу $\mathscr{S}_{m}$ (началам путей поднятой петли сопоставляются их концы). Обратно, если задан гомоморфизм $\mu: \pi_{1}\left(\mathbb{C P} \backslash\left\{q_{1}, \ldots, q_{n}\right\}, q_{0}\right) \rightarrow \mathscr{S}_{m}$, то он определяет некоторое накрытие $f: C \rightarrow \mathbb{C P}^{1}$, монодромия которого равна $\mu$.

Фундаментальная группа $\pi_{1}\left(\mathbb{C P} \backslash\left\{q_{1}, \ldots, q_{n}\right\}, q_{0}\right)$ является свободной группой и порождается петлями $\gamma_{1}, \ldots, \gamma_{n}$ следующего вида. Каждая петля $\gamma_{i}$ состоит из пути $l_{i}$ с началом в точке $q_{0}$ и концом в точке $q_{i}^{\prime}$, близкой к точке $q_{i}$, обхода в положительном направлении (относительно комплексной ориентации на $\left.\mathbb{C P}^{1}\right)$ по окружности $\Gamma_{i}$ малого радиуса с центром в точке $q_{i}, q_{i}^{\prime} \in \Gamma$, и возврата в точку $q_{0}$ вдоль пути $l_{i}$ в обратном направлении; петли $\gamma_{i}$ и $\gamma_{j}$ имеют только одну общую точку - точку $q_{0}$, и произведение $\gamma_{1} \ldots \gamma_{n}$ равно $\mathbf{1}$ в группе $\pi_{1}\left(\mathbb{C P} \backslash\left\{q_{1}, \ldots, q_{n}\right\}, q_{0}\right)$. Такой набор порождающих элементов называется хорошим геометрическим базисом группы $\pi_{1}\left(\mathbb{C P} \backslash\left\{q_{1}, \ldots, q_{n}\right\}, q_{0}\right)$.

Если выбран хороший геометрический базис $\gamma_{1}, \ldots, \gamma_{n}$, то монодромия $\mu$ определяется набором элементов $\sigma_{1}=\mu\left(\gamma_{1}\right), \ldots, \sigma_{n}=\mu\left(\gamma_{n}\right) \in \mathscr{S}_{m}$, который удовлетворяет условию: произведение $\sigma_{1} \ldots \sigma_{n}$ равно $1 \in \mathscr{S}_{m}$.

Набор $\left\{\sigma_{1}, \ldots, \sigma_{n}\right\}$ зависит от выбора базиса $\gamma_{1}, \ldots, \gamma_{n}$. Если $\left\{\sigma_{1}^{\prime}, \ldots, \sigma_{n}^{\prime}\right\}-$ набор, соответствующий другому хорошему геометрическому базису $\gamma_{1}^{\prime}, \ldots, \gamma_{n}^{\prime}$, то от набора $\left\{\sigma_{1}, \ldots, \sigma_{n}\right\}$ можно перейти к набору $\left\{\sigma_{1}^{\prime}, \ldots, \sigma_{n}^{\prime}\right\}$ с помощью так называемых преобразований Гурвица, т. е. преобразований, которые заменяют в $\left\{\sigma_{1}, \ldots, \sigma_{n}\right\}$ некоторые два соседних элемента $\left(\sigma_{i}, \sigma_{i+1}\right)$ на $\left(\sigma_{i+1}, \sigma_{i+1}^{-1} \sigma_{i} \sigma_{i+1}\right)$ 
или $\left(\sigma_{i} \sigma_{i+1} \sigma_{i}^{-1}, \sigma_{i}\right)$ и сохраняют все остальные. Два набора называются Гурвиц-эквивалентными, если один из них может быть получен из другого с помощью конечного числа преобразований Гурвица. Отметим, что преобразования Гурвица сохраняют неизменным произведение $\sigma_{1} \ldots \sigma_{n}$, которое в нашем случае равно 1.

Накрытие $f: C \rightarrow \mathbb{C P}^{1}$ называется общим накрытием проективной прямой, если все $\sigma_{i}$ являются транспозициями и порождают всю группу $\mathscr{S}_{m}$. Так как $\sigma_{1} \ldots \sigma_{n}=1$, то число точек ветвления общего накрытия должно быть четным. Пространство $\mathfrak{H}_{m, 2 k}$ общих накрытий проективной прямой степени $m$, разветвленных в $n=2 k$ точках, было исследовано А. Гурвицом в 1891 г. в [1]. В частности, им было доказано, что пространство $\mathfrak{H}_{m, 2 k}$ является связным. Связность пространства $\mathfrak{H}_{m, 2 k}$ следует из того, что любые два набора транспозиций $\left\{\sigma_{1}, \ldots, \sigma_{n}\right\}$ и $\left\{\sigma_{1}^{\prime}, \ldots, \sigma_{n}^{\prime}\right\}$ являются Гурвиц-эквивалентными, если каждый из них порождает всю группу $\mathscr{S}_{m}$ и $\sigma_{1} \ldots \sigma_{n}=\sigma_{1}^{\prime} \ldots \sigma_{n}^{\prime}=\mathbf{1}$.

В общем случае если $X \subset \mathbb{C P}^{N}$ - проективное алгебраическое многообразие, $\operatorname{dim} X=n$, то $X$ можно спроектировать на $\mathbb{C P}^{n}$ и, тем самым, представить его в виде конечнолистного накрытия $f: X \rightarrow \mathbb{C P}^{n}$, разветвленного вдоль некоторого подмногообразия $Y \subset \mathbb{C P}^{n}, \operatorname{dim} Y=n-1$. Продолжая этот процесс, мы можем спроектировать $Y$ на $\mathbb{C P}^{n-1}$ и т. д.

Как и в одномерном случае, фундаментальная группа $\pi_{1}\left(\mathbb{C P}^{n} \backslash Y, p\right)$ дополнения к $Y$, где $p \in \mathbb{C P}^{n} \backslash Y$, действует на слое $f^{-1}(p)$. Тем самым определен гомоморфизм $\mu=\mu_{f}: \pi_{1}\left(\mathbb{C P}^{n} \backslash Y, p\right) \rightarrow \mathscr{S}_{m}$ (монодромия степени $\left.m=\operatorname{deg} f\right)$ в симметрическую группу $\mathscr{S}_{m}$. Монодромия $\mu$ определяется отображением $f$ однозначно с точностью до внутренних автоморфизмов симметрической группы.

Обратно, согласно теореме Грауэрта-Ремерта-Штейна [2] гомоморфизм $\mu: \pi_{1}\left(\mathbb{C P}^{n} \backslash Y, p\right) \rightarrow \mathscr{S}_{m}$, образ $\operatorname{Im} \mu$ которого транзитивно действует на множестве из $m$ элементов, является монодромией некоторого конечного морфизма $f: X \rightarrow \mathbb{C P}^{n}$ степени $\operatorname{deg} f=m$, где $X$ - некоторое нормальное неприводимое проективное алгебраическое многообразие.

Аналогичные результаты имеют место и в симплектической ситуации: любое четырехмерное симплектическое многообразие (с целочисленной симплектической формой) можно представить как разветвленное накрытие проективной плоскости, причем множество $\bar{H}$ (кривая Гурвица, определение которой будет дано ниже), в котором разветвлено это накрытие, обладает практически теми же свойствами, что и дивизор ветвления в алгебраическом случае (см. [3]). В частности, это накрытие определяет монодромию $\mu: \pi_{1}\left(\mathbb{C P}^{2} \backslash \bar{H}, p\right) \rightarrow \mathscr{S}_{m}$, где $m$ - степень накрытия. Обратно, если $\bar{H}$ имеет только комплексно-аналитические особенности и задан гомоморфизм $\mu: \pi_{1}\left(\mathbb{C P}^{2} \backslash \bar{H}, p\right) \rightarrow \mathscr{S}_{m}$, то можно построить симплектическое четырехмерное многообразие $X$ с аналитическими особенностями и конечное накрытие $f: X \rightarrow \mathbb{C P}^{2}$, разветвленное над $\bar{H}$, монодромия которого $\mu_{f}$ равна $\mu$ (см. 9 ).

Вышеизложенное объясняет важность исследования фундаментальных групп дополнений к гиперповерхностям в проективном пространстве и к кривым Гурвица в $\mathbb{C P}^{2}$. Кроме того, заметим, что согласно теореме Лефшеца о фундаментальных группах гиперплоских сечений исследование фундаментальных групп дополнений к гиперповерхностям в проективном пространстве 
также сводится к изучению фундаментальных групп дополнений к плоским алгебраическим кривым.

Исследование фундаментальных групп дополнений к плоским алгебраическим кривым было начато в тридцатые годы прошлого столетия Э. Р. ван Кампеном и О. Зариским. Так, теорема, носящая их имя, дает метод вычисления фундаментальных групп $\pi_{1}\left(\mathbb{C P}^{2} \backslash C\right)$ дополнений к кривым $C \subset \mathbb{C P}^{2}$, который состоит в следующем. Пусть pr: $\mathbb{C P}^{2} \rightarrow \mathbb{C P}^{1}$ - линейная проекция на проективную прямую $\mathbb{C P}^{1}$ с центром в некоторой точке $p_{\infty} \subset \mathbb{C P}^{2} \backslash C$. Ограничение проекции pr на кривую $C$ является конечным накрытием прямой $\mathbb{C P}^{1}$ степени $m=\operatorname{deg} C$, разветвленным над конечным числом точек, скажем, $q_{1}, \ldots, q_{n}$. Если выбраны точка $q_{0} \in \mathbb{C P}^{1} \backslash\left\{q_{1}, \ldots, q_{n}\right\}$ и точка $p_{0} \in \operatorname{pr}^{-1}\left(q_{0}\right) \backslash C$, то теорема Зариского-ван Кампена утверждает, что $\pi_{1}\left(\mathbb{C P}^{2} \backslash C, p_{0}\right)$ порождается петлями, лежащими в слое $\mathrm{pr}^{-1}\left(q_{0}\right)$ и являющимися обходами вокруг точек $p_{i} \in \operatorname{pr}^{-1}\left(q_{0}\right) \cap C$, а соотношения между ними дает брэйд-монодромия кривой $C$ (см. $\S 7)$. Напомним ее определение.

Выберем некоторый слой $R_{\infty}$ проекции $\mathrm{pr}$, общий относительно кривой $C$, и положим $\mathbb{C}^{2}=\mathbb{C P}^{2} \backslash R_{\infty}$ и $\mathbb{C}^{1}=\mathbb{C P}^{1} \backslash \operatorname{pr}\left(R_{\infty}\right)$. После этого выберем открытый диск $D_{1} \subset \mathbb{C}^{1}$ такой, что $q_{1}, \ldots, q_{n} \in D_{1}$. Не ограничивая общности, можем считать, что $q_{0} \in \partial D_{1}$. Затем выбираем открытый диск $D_{2}$ такой, что $\operatorname{pr}^{-1}\left(\bar{D}_{1}\right) \cap C \subset \bar{D}_{1} \times D_{2} \subset \mathbb{C}^{2}$. Каждая петля $\gamma \subset \bar{D}_{1}$ с начальной точкой $q_{0}$ может быть поднята в $C$ с помощью проекции pr. В результате подъема получаем $m$ путей, начинающихся и заканчивающихся в точках $p_{1}, \ldots, p_{m}$. Эти пути определяют косу $b(\gamma) \in \operatorname{Br}\left[\bar{D}_{2},\left\{p_{1}, \ldots, p_{m}\right\}\right]=\operatorname{Br}_{m}$. Сопоставление каждой петле $\gamma \subset \bar{D}_{1}$ косы $b(\gamma)$ задает гомоморфизм $b: \pi_{1}\left(\mathbb{C P} \backslash\left\{q_{1}, \ldots, q_{n}\right\}, q_{0}\right) \rightarrow \mathrm{Br}_{m}$, который называется брэйд-монодромией кривой $C$ относительно проекции $\mathrm{pr}$.

Фундаментальная группа $\pi_{1}\left(\bar{D}_{1} \backslash\left\{q_{1}, \ldots, q_{n}\right\}, q_{0}\right)$ является свободной группой. Как и в случае фундаментальной группы дополнения к конечному числу точек на проективной прямой, для $\pi_{1}\left(\bar{D}_{1} \backslash\left\{q_{1}, \ldots, q_{n}\right\}, q_{0}\right)$ можно также ввести понятие хорошего геометрического базиса $\gamma_{1}, \ldots, \gamma_{n}$, которое отличается от приведенного выше только тем, что произведение $\gamma_{1} \ldots \gamma_{n}$ в группе $\pi_{1}\left(\bar{D}_{1} \backslash\left\{q_{1}, \ldots, q_{n}\right\}, q_{0}\right)$ равно не $\mathbf{1}$, а границе $\partial \bar{D}_{1}$ диска $\bar{D}_{1}$ (с обходом против часовой стрелки).

Если выбран хороший геометрический базис $\gamma_{1}, \ldots, \gamma_{n}$ в фундаментальной группе $\pi_{1}\left(\bar{D}_{1} \backslash\left\{q_{1}, \ldots, q_{n}\right\}, q_{0}\right)$, то брэйд-монодромия кривой $C$ относительно проекции $\mathrm{pr}$ определяется набором элементов $b_{1}=b\left(\gamma_{1}\right), \ldots, b_{n}=b\left(\gamma_{n}\right) \in \mathrm{Br}_{m}$. Этот набор обладает следующим свойством: произведение $b_{1} \ldots b_{n}$ равно $\Delta_{m}^{2}=$ $b\left(\partial \bar{D}_{1}\right)$, где $\Delta_{m}^{2}$ - порождающий элемент центра группы $\mathrm{Br}_{m}$.

Набор кос $\left\{b_{1}, \ldots, b_{n}\right\}$ зависит от выбора хорошего геометрического базиса $\gamma_{1}, \ldots, \gamma_{n}$. Если $\left\{b_{1}^{\prime}, \ldots, b_{n}^{\prime}\right\}$ - набор, соответствующий другому хорошему геометрическому базису $\gamma_{1}^{\prime}, \ldots, \gamma_{n}^{\prime}$, то, как и выше, от набора $\left\{b_{1}, \ldots, b_{n}\right\}$ можно перейти к набору $\left\{b_{1}^{\prime}, \ldots, b_{n}^{\prime}\right\}$ с помощью преобразований Гурвица. Класс Гурвиц-эквивалентных наборов, которому принадлежит набор $\left\{b\left(\gamma_{1}\right), \ldots, b\left(\gamma_{n}\right)\right\}$, называется разложением на множители брэйд-монодромии кривой $C$ относительно проекции $\mathrm{pr}$.

Применение разложений на множители брэйд-монодромии плоской алгебраической кривой для изучения топологии вложения этой кривой в $\mathbb{C P}^{2}$ впер- 
вые было использовано в работах О. Кизини [4], [5] и интенсивно использовалось Б. Мойшезоном и М. Тайхер [6]-[13] в восьмидесятые годы прошлого столетия при исследовании свойств дискриминантных кривых общих накрытий плоскости. В этих работах разложения на множители брэйд-монодромии рассматривались как настоящие разложения на множители элемента $\Delta_{m}^{2}$ с точностью до преобразований Гурвица (т. е. в полном соответствии с приведенным выше определением). В данном обзоре предлагается рассматривать Гурвицэквивалентные разложения на множители брэйд-монодромии как элементы некоторых полугрупп над группами кос. Этот подход на наш взгляд является более естественным и инвариантным. Чтобы объяснить естественность этого подхода, отметим, что при изучении топологии вложения плоских алгебраических кривых в $\mathbb{C P}^{2}$ можно “забыть про их алгебраическое происхождение" и рассматривать их как кривые Гурвица.

Понятие кривых Гурвица на проективной плоскости $\mathbb{C P}^{2}$ относительно линейной проекции pr: $\mathbb{C P}^{2} \rightarrow \mathbb{C P}^{1}$ было введено в [13] и является естественным обобщением понятия плоских алгебраических кривых (в [13] кривые Гурвица называются "полуалгебраическими кривыми", но так как в симплектическом случае приходится рассматривать кроме комплексно-аналитических особенностей также и другие особые точки, то термин “кривые Гурвица" представляется нам более удачным).

Грубо говоря, (топологическая) кривая Гурвица степени $m$ - это образ $\bar{H}$ гладко (или даже непрерывно) погруженной в линейное расслоение над $\mathbb{C P}^{1}$ (или над диском) замкнутой ориентированной римановой поверхности, который ведет себя относительно проекции pr так же, как и алгебраические кривые, т. е. число точек пересечения кривой Гурвица с каждым слоем расслоения меньше или равно $m$ и существует лишь конечное число слоев, число точек пересечения с которыми строго меньше $m$. Точки, в которых кривая $\bar{H}$ пересекается со слоями расслоения не трансверсально, называются критическими точками кривой $\bar{H}$. По определению кривой Гурвица, в окрестности каждой своей критической точки кривая $\bar{H}$ гомеоморфна конусу $C(b)=\{(r u, r v) \mid 0 \leqslant$ $r \leqslant 1,(u, v) \in b\}$ в бидиске $D=D_{1} \times D_{2}$, где $D_{1}=\left\{u \in \mathbb{C}^{1}|| u \mid \leqslant 1\right\}$, $D_{2}=\left\{v \in \mathbb{C}^{1}|| v \mid \leqslant 1\right\}$, а $b$ - замкнутая коса в $\partial D_{1} \times D_{2}$ и проекция pr совпадает с проекцией $D_{1} \times D_{2} \rightarrow D_{1}$. Однако в настоящем обзоре мы ограничиваемся рассмотрением кривых Гурвица только с критическими точками, которые локально являются либо комплексно-аналитическими особыми точками, либо так называемыми отрицательными ноудами (по определению, отрицательный ноуд - это критическая точка кривой Гурвица $\bar{H}$, в некоторой окрестности которой $\bar{H}$ является объединением двух гладких сечений проекции $\mathrm{pr}$, имеющих индекс пересечения в критической точке, равный -1 , и трансверсально пересекающих слой с положительным индексом пересечения).

Как и в алгебраическом случае, с каждой кривой Гурвица можно связать ассоциированное с ней разложение на множители брэйд-монодромии.

Переход от плоских алгебраических кривых к кривым Гурвица дает возможность рассматривать их с точностью до $H$-изотопии (т. е. изотопии в классе кривых Гурвица). С одной стороны, это не меняет топологию дополнения к этим кривым. С другой стороны, это делает конструкцию более мягкой, дает 
возможность разрезать кривые на части, приклеивать их друг к другу (“делать хирургию"). Склеивание двух кривых Гурвица над диском дает опять кривую Гурвица над диском, и это можно рассматривать как операцию умножения. Мы получаем на множестве кривых Гурвица над диском, рассматриваемых с точностью до эквивалентности ( $H$-изотопии), структуру полугруппы. Опишем эту важную с идейной и практической точки зрения конструкцию в более простой ситуации, когда конечное накрытие диска не вложено в линейное расслоение, а рассматривается само по себе.

Забыв о комплексной структуре на римановой поверхности $S$ степени $m$ над замкнутым диском $D=\{u \in \mathbb{C}|| u \mid \leqslant 1\}$, рассмотрим ее как ориентированную (не обязательно связную) поверхность $S$ (с краем) вместе с собственным непрерывным накрытием $f: S \rightarrow D$ степени $\operatorname{deg} f=m$, не разветвленным над $D \backslash\left\{p_{1}, \ldots, p_{n}\right\}$, где $p_{1}, \ldots, p_{n} \in D \backslash \partial D$ - некоторый набор, состоящий из $n=n(f)$ точек. Кроме того, в каждом накрытии $(S, f)$ упорядочим точки $q_{1}, \ldots, q_{m}$, принадлежащие прообразу $f^{-1}\left(p_{0}\right)$, где $p_{0} \in \partial D$ - некоторая фиксированная точка (пусть, для определенности, $p_{0}=-i \in \partial D$ ). Такие накрытия будем называть накрытиями с упорядоченными листами или с оснащением. Скажем, что оснащенные накрытия $\left(S^{\prime}, f^{\prime}\right)$ и $\left(S^{\prime \prime}, f^{\prime \prime}\right)$ эквивалентны, если существуют гомеоморфизмы $\psi: D \rightarrow D$ и $\varphi: S^{\prime} \rightarrow S^{\prime \prime}$ такие, что

(i) гомеоморфизм $\psi$ неподвижен на границе $\partial D$;

(ii) $\varphi\left(q_{i}^{\prime}\right)=q_{i}^{\prime \prime}$ для $i=1, \ldots, m$;

(iii) $\psi \circ f^{\prime}=f^{\prime \prime} \circ \varphi$.

Обозначим через $\mathscr{R}_{m}$ множество классов оснащенных накрытий диска степени $m$ относительно этой эквивалентности.

На множестве $\mathscr{R}_{m}$ можно ввести структуру полугруппы следующим образом. Пусть $\left(S_{1}, f_{1}\right)$ и $\left(S_{2}, f_{2}\right)$ - два оснащенных накрытия степени $m$. Выберем два непрерывных сохраняющих ориентацию вложения $\varphi_{j}: D \rightarrow D, j=1,2$, диска $D$ в себя, оставляющих точку $p_{0}$ на месте и таких, что

(i) образ $\varphi_{1}(D)=\{u \in D \mid \operatorname{Re} u \geqslant 0\}$ - это правый полукруг и $\varphi_{1}(\{u \in \partial D \mid \operatorname{Re} u \leqslant 0\})=\{u \in D \mid \operatorname{Re} u=0\}$ - вертикальный диаметр;

(ii) $\varphi_{2}(D)=\{u \in D \mid \operatorname{Re} u \leqslant 0\}$ - это левый полукруг и $\varphi_{2}(\{u \in \partial D \mid \operatorname{Re} u \geqslant 0\})=\{u \in D \mid \operatorname{Re} u=0\}$.

Отождествим точки, принадлежащие множествам $f_{1}^{-1}\left(p_{0}\right)$ и $f_{2}^{-1}\left(p_{0}\right)$, согласно выбранным выше нумерациям этих точек и после этого по непрерывности отождествим точки, принадлежащие $m$ путям $f_{1}^{-1}(\{u \in \partial D \mid \operatorname{Re} u \leqslant 0\})$ на $S_{1}$, с точками, принадлежащими $m$ путям $f_{2}^{-1}(\{u \in \partial D \mid \operatorname{Re} u \geqslant 0\})$ на $S_{2}$, так, чтобы совпадали образы всех отождествленных точек при отображениях $\varphi_{1} \circ f_{1}$ и $\varphi_{2} \circ f_{2}$. С помощью этого отождествления мы можем склеить поверхности $S_{1}$ и $S_{2}$ вдоль этих $m$ путей и получить оснащенное накрытие $(S, f)$, где $f(q)=\varphi_{1}\left(f_{1}(q)\right)$, если $q \in S_{1}$, и $f(q)=\varphi_{2}\left(f_{2}(q)\right)$, если $q \in S_{2}$. Назовем полученное накрытие $(S, f)$ произведением оснащенных накрытий $\left(S_{1}, f_{1}\right)$ и $\left(S_{2}, f_{2}\right)$ (обозначение: $\left.(S, f)=\left(S_{1}, f_{1}\right) \cdot\left(S_{2}, f_{2}\right)\right)$. Легко видеть, что так определенное произведение задает на $\mathscr{R}_{m}$ структуру некоммутативной полугруппы.

Легко видеть, что полугруппа $\mathscr{R}_{m}$ порождается оснащенными накрытиями $(S, f)$, которые являются накрытиями диска $D$ с только одной точкой ветвления $p_{1}$. Такие накрытия однозначно (с точностью до эквивалентности) опре- 
деляются своей глобальной монодромией $\sigma_{f}=\mu(\partial D) \in \mathscr{S}_{m}$, где $\mu=\mu_{f}-$ монодромия оснащенного накрытия $(S, f)$. Следовательно, число порождающих элементов равно $m$ ! . Обозначим порождающий элемент полугруппы $\mathscr{R}_{m}$, соответствующий накрытию $(S, f)$ с одной точкой ветвления, через $x_{\sigma_{f}}$. Простая проверка показывает, что в полугруппе $\mathscr{R}_{m}$ выполнены следующие соотношения:

$$
x_{\sigma_{1}} \cdot x_{\sigma_{2}}=x_{\sigma_{2}} \cdot x_{\sigma_{2}^{-1} \sigma_{1} \sigma_{2}}, \quad x_{\sigma_{1}} \cdot x_{\sigma_{2}}=x_{\sigma_{1} \sigma_{2} \sigma_{1}^{-1}} \cdot x_{\sigma_{1}},
$$

а также $x_{\sigma_{1}} \cdot x_{1}=x_{\sigma_{1}}$ и $x_{1} \cdot x_{\sigma_{2}}=x_{\sigma_{2}}$ для всех $\sigma_{1}, \sigma_{2} \in \mathscr{S}_{m}$, и можно показать, что эти соотношения являются определяющими.

Непосредственно проверяется, что если оснащенное накрытие $(S, f)$ равно элементу $x_{\sigma_{1}} \cdot \ldots \cdot x_{\sigma_{n}}$ в полугруппе $\mathscr{R}_{m}$, то ее глобальная монодромия $\sigma_{f}=$ $\mu(\partial D)$ равна произведению $\sigma_{1} \ldots \sigma_{n}$, и, очевидно, сопоставление каждому накрытию его глобальной монодромии является гомоморфизмом из полугруппы $\mathscr{R}_{m}$ в группу $\mathscr{S}_{m}$. Обозначим этот гомоморфизм через $\alpha: \mathscr{R}_{m} \rightarrow \mathscr{S}_{m}$.

Перенумерации листов на оснащенных накрытиях определяют действие группы $\mathscr{S}_{m}$ на полугруппе $\mathscr{R}_{m}$. А именно, элемент $\sigma_{0} \in \mathscr{S}_{m}$ действует на порождающих элементах $x_{\sigma}$ по следующему правилу: $x_{\sigma} \mapsto x_{\sigma_{0}^{-1} \sigma \sigma_{0}}$. Это действие определяет гомоморфизм $\lambda: \mathscr{S}_{m} \rightarrow \operatorname{Aut}\left(\mathscr{R}_{m}\right)$. Очевидно, что если забыть об упорядочении листов, то орбиты действия группы $\mathscr{S}_{m}$ на полугруппе $\mathscr{R}_{m}$ взаимно однозначно соответствуют классам эквивалентных накрытий диска степени $m$.

Очевидно также, что ядро $\operatorname{ker} \alpha=\mathscr{R}_{m, \mathbf{1}}=\left\{(S, f) \in \mathscr{R}_{m} \mid \sigma_{f}=\mathbf{1}\right\}$ является подполугруппой в $\mathscr{R}_{m}$. Если диск $D$ вложен в $\mathbb{C P}^{1}$, то элементы полугруппы $\mathscr{R}_{m, \mathbf{1}}$ - это те накрытия $f: S \rightarrow D$, для которых существует продолжение накрытия $f$ до накрытия $\tilde{f}: \widetilde{S} \rightarrow \mathbb{C P}^{1}$, неразветвленного над $\mathbb{C P}^{1} \backslash D$.

Аналогичные полугруппы появляются также и в других контекстах: кривые Гурвица, пучки Лефшеца в симплектической геометрии и, более общо, расслоения над $\mathbb{C P}^{1}$ (или над диском), общий слой и типы вырожденных слоев которых фиксированы. Более точно, во всех этих случаях появляется некоторый алгебраический объект: полугруппа и ее гомоморфизм в некоторую, естественным образом определяемую задачей, группу, причем эта группа действует на полугруппе и это действие согласовано с гомоморфизмом. Такой объект мы называем nолугруппой над группой. Элементы этих полугрупп естественным образом взаимно однозначно соответствуют изучаемым геометрическим объектам с точностью до некоторой эквивалентности. Все это мотивирует отдельное изучение полугрупп над группами. В $\S 2$ настоящего обзора дано общее определение полугрупп над группами, приведены основные конструкции и исследованы некоторые их функториальные свойства в наиболее важном для нас случае, когда эти полугруппы являются полугруппами разложений на множители над группами. Третий параграф посвящен описанию некоторых свойств полугрупп разложений на множители над группами кос, описанию множества групп, ассоциированных с элементами этих полугрупп, а также в этом параграфе исследуются и другие инварианты, такие как модули и многочлены Александера групп, ассоциированных с элементами этих полугрупп. 
В заключение введения скажем несколько слов о содержании других параграфов настоящего обзора. В $\S 1$ даются определения кривых Гурвица, лежащих на поверхностях Хирцебруха $F_{N}$, а также напоминаются определения псевдоголоморфных кривых и симплектических особых поверхностей.

В $§ 4$ вводится понятие разложения на множители брэйд-монодромии кривой Гурвица и псевдоголоморфной кривой в $\mathbb{C P}^{2}$ как элемента полугруппы разложений на множители над группой кос, дается набросок доказательства того, что каждое разложение на множители с множителями, являющимися брэйд-монодромиями ростков алгебраических особых точек, может быть реализовано как разложение на множители брэйд-монодромии некоторой алгебраической кривой над диском.

Пятый параграф посвящен понятию $H$-изотопии и обсуждению связи между разложениями на множители брэйд-монодромии и вопросами о $H$-изотопности кривых Гурвица и симплектической изотопности псевдоголоморфных кривых. В этом параграфе также дано определение полугруппы оснащенных кривых Гурвица над диском. В $\S 6$ исследован вопрос о регулярной гомотопности кривых Гурвица и псевдоголоморфных кривых. Регулярная гомотопия - это более слабое отношение эквивалентности по сравнению с $H$-изотопией. Регулярная гомотопия состоит из $H$-изотопий и таких деформаций кривой Гурвица, при которых одна из ветвей кривой может “наползать" на другую и в результате могут возникать пары дополнительных особых точек и каждая пара состоит из отрицательного и положительного ноуда.

Седьмой параграф посвящен описанию множества фундаментальных групп дополнений к "аффинным" кривым Гурвица. В этом параграфе также обсуждается проблема Зариского финитной аппроксимируемости фундаментальных групп дополнений к плоским алгебраическим кривым. Из-за ограниченности объема данного обзора пришлось опустить примеры конкретного вычисления фундаментальных групп дополнений к плоским алгебраическим кривым, основанного на вычислении разложений на множители брэйд-монодромии этих кривых. Эти примеры можно найти в работах Б. Мойшезона и М. Тайхер (cм. [6]-[14]).

В $\S 8$ рассматриваются конечнолистные накрытия проективной плоскости, разветвленные вдоль алгебраических кривых, и, в частности, общие накрытия плоскости, а также обсуждаются результаты, относящиеся к гипотезе Кизини о единственности общего накрытия плоскости степени $\geqslant 5$, разветвленного над данной каспидальной кривой, и возможности использовать брэйд-монодромные инварианты кривых ветвления общих накрытий в качестве инвариантов, различающих связные компоненты пространства модулей алгебраических поверхностей. В $\S 9$ обсуждаются аналогичные проблемы, но уже в симплектическом случае: обсуждается возможность использования брэйд-монодромных инвариантов кривых (Гурвица) ветвления общих накрытий $\mathbb{C P}^{2}$ симплектическими четырехмерными многообразиями в качестве инвариантов, различающих эти многообразия с точностью до симплектоморфизма.

Десятый параграф посвящен циклическим накрытиям проективной плоскости, разветвленным вдоль кривой Гурвица и, возможно, вдоль некоторой 
прямой; вычисляется первое число Бетти таких накрытий в терминах корней многочлена Александера кривой ветвления.

\section{$\S 1$. Кривые Гурвица на поверхностях Хирцебруха, псевдоголоморфные кривые и симплектические поверхности}

1.1. Определение кривых Гурвица. Как отмечалось во введении, кривые Гурвица являются обобщением понятия алгебраических кривых, лежащих в расслоениях над проективной прямой $\mathbb{C P}^{1}$ со слоем "проективная прямая". Таких расслоений существует счетное число - это поверхности Хирцебруха $F_{N}$.

Пусть $F_{N}$ - относительно минимальная рациональная линейчатая поверхность (поверхность Хирцебруха), $N \geqslant 1$, pr: $F_{N} \rightarrow \mathbb{C P}^{1}-$ ее линейчатая структура, $R_{p}$ - слой проекции $\mathrm{pr}$ над точкой $p \in \mathbb{C P}^{1}$ и $E_{N}-$ исключительное сечение, $E_{N}^{2}=-N$. По определению образ $\bar{H}=f(\Sigma) \subset F_{N}$ гладкого отображения $f: \Sigma \rightarrow F_{N} \backslash E_{N}$ ориентированной замкнутой вещественной поверхности $\Sigma$ называется кривой Гурвица (в $F_{N}$ ) степени $m$, если существует конечное подмножество $Z \subset \bar{H}$ такое, что:

(i) $f$ является вложением поверхности $\Sigma \backslash f^{-1}(Z)$ и для каждой точки $s \notin Z$ образ $\bar{H}$ и слой $R_{\operatorname{pr}(s)}$ проекции pr пересекаются в точке $s$ трансверсально с положительным индексом пересечения;

(ii) для каждой точки $s \in Z$ существует такая окрестность $U \subset F_{N}$ точки $s$, в которой $\bar{H} \cap U$ является комплексно-аналитической кривой и комплексная ориентация на $\bar{H} \cap U \backslash\{s\}$ совпадает с ориентацией, индуцированной ориентацией на $\Sigma$ с помощью отображения $f$;

(iii) ограничение проекции pr на $\bar{H}$ является конечным отображением степени $m$.

ЗАмечАнИЕ 1.1. Условие $\bar{H} \subset F_{N} \backslash E_{N}$ является довольно искусственным. Оно необходимо для того, чтобы иметь возможность сопоставить каждой кривой Гурвица $\bar{H}$ разложение на множители ее брэйд-монодромии (см. $\S 4$ ).

Кривая Гурвица $\bar{H}$ называется неприводимой, если множество $\bar{H} \backslash M$ связно для любого конечного множества $M \subset \bar{H}$, и мы будем говорить, что кривая Гурвица $\bar{H}$ состоит из $k$ неприводимых компонент, если

$$
k=\max \#\{\text { связные компоненты кривой } \bar{H} \backslash M\},
$$

где максимум взят по всем конечным множествам $M \subset \bar{H}$.

Для каждой кривой Гурвица $\bar{H}$ существует единственное минимальное множество $Z \subset \bar{H}$, которое удовлетворяет условиям из определения кривых Гурвица. Обозначим его через $\operatorname{Crit} \bar{H}$ и будем называть множеством критических точек кривой Гурвица $\bar{H}$, а его образ $\operatorname{pr}(\operatorname{Crit} \bar{H}) \subset \mathbb{C P}^{1}$ будем называть множеством критических значений кривой $\bar{H}$. Точки подмножества $\operatorname{Sing} \bar{H} \subset \operatorname{Crit} \bar{H}$, в которых $\bar{H}$ не является гладким подмногообразием проективной плоскости, называются особыми.

Кривая Гурвица $\bar{H}$ называется каспидальной, если для каждой точки $s \in$ Crit $\bar{H}$ существуют окрестность $U \subset F_{N}$ точки $s$ и локальные аналитические координаты $u, v$ в $U$ такие, что 
(iv) проекция $\operatorname{pr}_{\mid U}$ совпадает с отображением $(u, v) \mapsto u$;

(v) кривая $\bar{H} \cap U$ задается уравнением $v^{2}=u^{k}$.

Кривая Гурвица называется обыкновенной каспидальной, если в условии (v) имеет место неравенство $k \leqslant 3$ для всех $s \in \operatorname{Crit} \bar{H}$, и - нодальной, если $k \leqslant 2$. Если $\bar{H}$ является обыкновенной каспидальной кривой Гурвица, то все ее критические точки являются либо (при $k=1$ ) простыми точками касания со слоями проекции $\mathrm{pr}$, либо (при $k=2$ ) невырожденными двойными точками (для краткости будем называть такие точки ноудами), либо (при $k=3$ ) обыкновенными точками возврата (для краткости - обыкновенными каспами).

В симплектической геометрии часто возникает необходимость рассматривать также и кривые Гурвица $\bar{H}$ с отрицательными ноудами, т. е. п. (ii) определения кривых Гурвица заменяется на

$\left(\right.$ ii' $^{\prime}$ для каждого $s \in Z$ существует такая окрестность $U \subset F_{N}$ точки $s$, что $\bar{H}$ либо локально задается аналитическим уравнением в окрестности $U$, либо $\bar{H} \cap U$ состоит из двух гладких ветвей, пересекающихся трансверсально в точке $s$ с индексом пересечения, равным -1 , и каждая ветвь кривой $\bar{H} \cap U$ пересекает слой $R_{\operatorname{pr}(s)}$ в точке $s$ трансверсально с индексом пересечения, равным +1 .

Легко показать (лемма 1.1 в [15]), что приведенное выше определение кривых Гурвица эквивалентно следующему определению. А именно, пусть $\mathbb{C}_{i}^{2}-$ две копии аффинной плоскости $\mathbb{C}^{2}, i=1,2$, с координатами $\left(u_{i}, v_{i}\right), u_{2}=1 / u_{1}$ и $v_{2}=v_{1} / u_{1}^{N}$, которые покрывают $F_{N} \backslash E_{N}$ так, что $\mathrm{pr}$ задана формулами $\left(u_{i}, v_{i}\right) \rightarrow u_{i}$ в картах $\mathbb{C}_{i}^{2}$. Множество $\bar{H} \subset F_{N} \backslash E_{N}$, замкнутое в $F_{N}$, называется кривой Гурвица степени $m$, если для $i=1,2$ множество $\bar{H} \cap \mathbb{C}_{i}^{2}$ совпадает с множеством решений уравнения

$$
F_{i}\left(u_{i}, v_{i}\right):=v_{i}^{m}+\sum_{j=0}^{m-1} c_{i, j}\left(u_{i}\right) v_{i}^{j}=0
$$

где

(j) $F_{i}\left(u_{i}, v_{i}\right)$ является $C^{\infty}$-гладкой комплекснозначной функцией в $\mathbb{C}_{i}^{2}$;

(jj) множество $\operatorname{Crit}_{i} \bar{H}=\left\{\left(u_{i, 1}, v_{i, 1}\right), \ldots,\left(u_{i, n_{i}}, v_{i, n_{i}}\right)\right\} \subset \mathbb{C}_{i}^{2}$, состоящее из точек таких, что $v_{i, j}$ является кратным корнем полиномиального уравнения

$$
v_{i}^{m}+\sum_{j=0}^{m-1} c_{i, j}\left(u_{i, j}\right) v_{i}^{j}=0,
$$

является конечным;

$(\mathrm{jjj})$ если $\left(u_{i, j}, v_{i, j}\right) \in \mathrm{Crit}_{i} \bar{H}$, то в окрестности точки $\left(u_{i, j}, v_{i, j}\right)$ (которую мы называем критической точкой кривой $\bar{H}$ ) множество $\bar{H}$ совпадает либо с множеством решений некоторого комплексно-аналитического уравнения, либо (после послойной гладкой, сохраняющей ориентацию замены координат в этой окрестности) с множеством решений уравнения $v^{2}-\bar{u}^{2}=0$ (отрицательный ноуд).

Очевидно, что Crit $\bar{H}=\operatorname{Crit}_{1} \bar{H} \cup \operatorname{Crit}_{2} \bar{H}$ и, не ограничивая общности (выбрав другое покрытие множества $F_{N} \backslash E_{N}$ картами $\left.\mathbb{C}_{i}^{2}, i=1,2\right)$, мы можем 
(и будем) считать, что Crit $\bar{H} \subset \mathbb{C}_{1}^{2}$. Кривую $H=\bar{H} \cap \mathbb{C}_{1}^{2}$ будем называть афбфинной кривой Гурвица, а слой $R_{\infty}=\operatorname{pr}^{-1}(\infty)$ над точкой $\infty=\mathbb{C P}^{1} \backslash \operatorname{pr}\left(\mathbb{C}_{1}^{2}\right)$ будем называть "бесконечно удаленным" слоем.

Пусть $\bar{H}$ - кривая Гурвица в $F_{1}$. Мы можем стянуть исключительную кривую $E_{1}$ в точку и получить проективную плоскость $\mathbb{C P}^{2}$. Обозначим это стягивание через $\sigma: F_{1} \rightarrow \mathbb{C P}^{2}$. При этом стягивании слои проекции pr переходят в прямые на $\mathbb{C P}^{2}$, проходящие через точку $p_{\infty}=\sigma\left(E_{1}\right)$. Образ $\sigma(\bar{H})$ кривой $\bar{H}$ будем называть кривой Гурвица в $\mathbb{C P}^{2}$ относителъно пучка прямых, проходящих через точку $p_{\infty}$ (или относительно линейной проекции с центром в точке $\left.p_{\infty}\right)$.

Отметим, что если коэффициенты $c_{i, j}\left(u_{i}\right)$ в уравнении (1) являются многочленами от переменной $u_{i}$ с комплексными коэффициентами, то $\bar{H}$ является алгебраической кривой. Обратно, если алгебраическая кривая $C \subset F_{N}$ не пересекается с исключительным сечением $E_{N}$, то $C$ является кривой Гурвица.

Понятие алгебраической кривой может быть обобщено следующим образом. А именно, кривая Гурвица $\bar{H} \subset F_{N}$ называется почти алгебраической кривой степени $m$, если найдется бидиск

$$
D_{r_{1}, r_{2}}=\left\{\left|u_{1}\right|<r_{1},\left|v_{1}\right|<r_{2}\right\} \subset \mathbb{C}_{1}^{2}
$$

такой, что коэффициенты $c_{1, j}\left(u_{1}\right)$ являются многочленами от переменной $u_{1}$ при $\left|u_{1}\right|<r_{1}$ (т. е. в бидиске $D_{r_{1}, r_{2}}$ кривая $\bar{H}$ совпадает с некоторой алгебраической кривой $C$ ), ограничение проекции $\operatorname{pr}$ на $\bar{H} \cap D_{r_{1}, r_{2}}$ является собственным отображением степени $m$ и $\bar{H}$ является объединением $m$ попарно непересекающихся сечений $H_{\infty, 1}, \ldots, H_{\infty, m}$ проекции $\operatorname{pr}$ над $\mathbb{C P}^{1} \backslash \operatorname{pr}\left(D_{r_{1}, r_{2}}\right)$.

1.2. Симплектические и почти комплексные структуры. Напомним, что дифференциальная 2-форма $\omega \in \Omega^{2}(X, \mathbb{R})$ на гладком $2 n$-мерном многообразии $X$ называется симплектической, если она невырождена, т. е. $\omega^{\wedge n}$ является формой объема на $X$, и замкнута. Пара $(X, \omega)$, где $\omega$ - симплектическая форма на $X$, называется симплектическим многообразием.

Согласно классической теореме Мозера, если две симплектические формы $\omega_{0}$ и $\omega_{1}$ на $X$ можно соединить гладким семейством $\omega_{t}$ симплектических форм из одного и того же класса когомологий, то существует изотопия $h_{t}$ такая, что $h_{0}=\mathrm{Id}$ и $h_{1}^{*}\left(\omega_{0}\right)=\omega_{1}$. Другими словами, в этом случае формы $\omega_{t}$ определяют на $X$ одну и ту же (с точностью до симплектоморфизма) симплектическую структуру.

Согласно теореме Дарбу локально каждое симплектическое многообразие симплектоморфно стандартной модели $\left(\mathbb{R}^{2 n}, \sum_{j=1}^{n} d x^{j} \wedge d y^{j}\right)$. Гладкое подмногообразие $Y \subset X$ симплектического многообразия $(X, \omega)$ называется симплектическим, если ограничение $\omega_{\mid Y}$ на $X$ формы $\omega$ является симплектической формой. Одна из версий теоремы Дарбу утверждает, что если $Y$ - симплектическое подмногообразие в $X$, то пара $Y \subset X$ локально симплектоморфна паре $\mathbb{R}^{2 k} \subset \mathbb{R}^{2 n}$ со стандартной симплектической формой.

Напомним, что почти комплексной структурой на гладком многообразии $X$ называется эндоморфизм $J: T X \rightarrow T X$ касательного расслоения $T X$ на $X$ 
такой, что $J^{2}=-$ Id. На каждом симплектическом многообразии $(X, \omega)$ существует особый класс почти комплексных структур, связанных с симплектической структурой. А именно, почти комплексная структура $J$ называется $\omega$-ручной, если выполнено следующее условие:

(i) $\omega(x, J x)>0$ для всех $x \in T X, x \neq 0$.

Если, кроме того, выполнено условие

(ii) $\omega(J x, J y)=\omega(x, y)$ для всех $x, y \in T X$,

то почти комплексная структура $J$ называется совместимой с $\omega$. Условия (i) и (ii) означают, что форма $\omega$ является мнимой частью эрмитовой метрики на комплексном векторном расслоении $(T X, J)$.

Важными примерами симплектических многообразий являются кэлеровы многообразия. В кэлеровом случае симплектическая форма - это кэлерова форма, а совместимая с ней почти комплексная структура - это обычная комплексная структура (которая в данном случае является интегрируемой).

Обобщением понятия комплексной кривой, лежащей на кэлеровом многообразии, является понятие псевдоголоморфной кривой. Псевдоголоморфной кривой на почти комплексном многообразии $(X, J)$ называется образ гладкого отображения $f: \Sigma \rightarrow X$ римановой поверхности $(\Sigma, j)$, дифференциал $d f$ которого в каждой точке является $\mathbb{C}$-линейным отображением.

Можно показать (см. [16]-[18]), что особые точки псевдоголоморфных кривых (т. е. образы точек, в которых $d f=0$ ) на почти комплексных четырехмерных многообразиях эквивалентны с точностью до $C^{1}$-замены координат особым точкам обычных комплексных кривых на комплексных поверхностях.

Отметим также, что согласно условию (i) ограничение формы $\omega$ на каждое $J$-инвариантное подпространство является положительной формой. Следовательно, псевдоголоморфные кривые на почти комплексном четырехмерном многообразии (в частности, комплексные кривые на кэлеровой поверхности) вне своих особых точек являются симплектическими поверхностями. Это наблюдение позволяет обобщить понятие симплектической поверхности в четырехмерном симплектическом многообразии на случай поверхностей с особыми точками.

Симплектической поверхностъю на симплектическом четырехмерном многообразии $(X, \omega)$ называется образ гладкого отображения $f: \Sigma \rightarrow X$ гладкой ориентированной поверхности $\Sigma$ такого, что дифференциал $d f$ вырожден лишь в конечном числе точек и, кроме того, выполнены следующие условия:

1) если $d f$ вырожден в точке $p \in \Sigma$, то в этой точке $d f=0$;

2) для каждой точки $p \in \Sigma$, в которой $d f \neq 0$, найдется такая окрестность $U \subset \Sigma$ этой точки, что $f(U)$ является симплектическим подмногообразием многообразия $(X, \omega)$;

$3)$ для каждой точки $p \in \Sigma$, в которой $d f=0$, найдутся окрестность $V \subset X$ точки $f(p)$ и почти комплексная структура $J$ на $V$, совместимая с $\omega_{\mid V}$, такие, что $f(\Sigma) \cap V$ является $J$-голоморфной кривой в $V$.

Симплектическая поверхность $\bar{H}=f(\Sigma)$ кроме особых точек $s=f(p)$, описанных в условии 3 ), может иметь и другие особые точки, в которых $\bar{H}$ не является многообразием, - это те точки, которые имеют более одного прообраза при отображении $f$ (в этих точках $\bar{H}$ локально является объединением нескольких 
своих неприводимых ветвей). Скажем, что особая точка $s=f(p)$ симплектической поверхности $\bar{H}$ является $J$-голоморфной, если найдутся окрестность $V \subset X$ точки $f(p)$ и почти комплексная структура $J$ на $V$, совместимая с $\omega_{\mid V}$, такие, что каждая ветвь поверхности $\bar{H}$ является $J$-голоморфной кривой в $V$. Отметим, что кроме $J$-голоморфных особых точек симплектическая поверхность $\bar{H}$ может иметь и другие особые точки. Можно показать (см. [18]), что для симплектической поверхности $\bar{H}$ в симплектическом многообразии $(X, \omega)$, все точки которой $J$-голоморфны, найдется почти комплексная структура $J$, совместимая с $\omega$, относительно которой $\bar{H}$ является псевдоголоморфной кривой.

1.3. Симплектические кривые Гурвица. Зафиксируем на поверхности Хирцебруха $F_{N}$ некоторую кэлерову форму $\omega$.

ПреДЛОЖенИЕ 1.1. Для каждой кривой Гурвица $\bar{H} \subset F_{N}$ существуют автоморбизм $h$ поверхности $F_{N}$ и почти комплексная структура $J$ на $F_{N}$, совместимая с $\omega$, такие, что $h(\bar{H})$ является одновременно кривой Гурвица и J-голоморфной кривой, если $\bar{H}$ не имеет отрицательных ноудов, и - симплектической поверхностъю в $\left(F_{N}, \omega\right)$, если $\bar{H}$ имеет отрицательные ноуды.

Доказательство этого предложения основано на следующем элементарном наблюдении (которое использовалось в похожей ситуации в [19]). Пусть $f$ : $\bar{D} \rightarrow \mathbb{C}$ - гладкая функция в окрестности диска $D=\{u \in \mathbb{C}|| u \mid<1\}$, и пусть $\Gamma_{f}=\left\{(u, f(u)) \in \mathbb{C}^{2} \mid u \in \bar{D}\right\}$ - ее график (являющийся поверхностью, вложенной в $\left.\mathbb{C}^{2}\right)$. Тогда для достаточно малого $\varepsilon>0$ график $\Gamma_{\varepsilon f}$ функции $\varepsilon f$ является симплектической поверхностью относительно стандартной симплектической формы.

Чтобы увидеть это, запишем симплектическую форму $\omega$ в комплексных координатах: $\omega=(i / 2)(d u \wedge d \bar{u}+d v \wedge d \bar{v})$. Ограничение на $\Gamma_{\varepsilon f}$ формы $\omega$ равно

$$
\frac{i}{2}\left(1+\varepsilon^{2}\left(\left|f_{u}^{\prime}\right|^{2}-\left|f_{\bar{u}}^{\prime}\right|^{2}\right)\right) d u \wedge d \bar{u} .
$$

Очевидно, что эта форма является положительной при достаточно малых $\varepsilon$.

Также полезно заметить, что комплексная кривая в $\mathbb{C}^{2}$, заданная уравнением $g(u, v)=0$, после перемасштабирования $(u, v) \mapsto(u, \varepsilon v)$ остается комплексной кривой. Аналогично, легко показать, что автоморфизм $h_{\varepsilon}$ поверхности $F_{N}$, заданный в картах $\mathbb{C}_{i}^{2}$ формулой $\left(u_{i}, v_{i}\right) \mapsto\left(u_{i}, \varepsilon v_{i}\right)$, при достаточно малых $\varepsilon$ переводит кривую Гурвица $\bar{H}$ в $h_{\varepsilon}(\bar{H})$, являющуюся симплектической поверхностью. Если кривая $\bar{H}$ не имеет отрицательных ноудов (т. е. все ее критические точки являются аналитическими), то найдется почти комплексная структура $J$ на $F_{N}$, совместимая с $\omega$, такая, что $h(\bar{H})$ является $J$-голоморфной кривой.

Упомянутый выше автоморфизм $h_{\varepsilon}$ поверхности $F_{N}$ называется nеремасштабированием.

\section{§ 2. Полугруппы разложений на множители}

В 4 , чтобы определить брэйд-монодромное разложение на множители, ассоциированное с кривой Гурвица, нам понадобятся некоторые вспомогательные определения и факты о разложениях на множители в произвольных группах. 
2.1. Полугруппы разложений на множители. Набор $(S, G, \alpha, \lambda)$, где $S$ - полугруппа, $G$ - группа и $\alpha: S \rightarrow G, \lambda: G \rightarrow \operatorname{Aut}(S)$ - гомоморфизмы, называется полугруппой $S$ над группой $G$, если для всех $s_{1}, s_{2} \in S$ имеет место

$$
s_{1} \cdot s_{2}=\lambda\left(\alpha\left(s_{1}\right)\right)\left(s_{2}\right) \cdot s_{1}=s_{2} \cdot \rho\left(\alpha\left(s_{2}\right)\right)\left(s_{1}\right),
$$

где $\rho(g)=\lambda\left(g^{-1}\right)$. Для данной пары полугрупп $\left(S_{1}, G_{1}, \alpha_{1}, \lambda_{1}\right)$ и $\left(S_{2}, G_{2}, \alpha_{2}, \lambda_{2}\right)$ над группами $G_{1}$ и $G_{2}$ соответственно пара гомоморфизмов $\left(h_{1}, h_{2}\right)$, где $h_{1}$ : $S_{1} \rightarrow S_{2}$ и $h_{2}: G_{1} \rightarrow G_{2}$, называется гомоморфизмом полугрупп над группами, если

(i) $h_{2} \circ \alpha_{S_{1}}=\alpha_{S_{2}} \circ h_{1}$,

(ii) $\lambda_{G_{2}}\left(h_{2}(g)\right)\left(h_{1}(s)\right)=h_{1}\left(\lambda_{G_{1}}(g)\right)(s)$ для всех $s \in S_{1}$ и всех $g \in G_{1}$.

Определенные ниже полугруппы разложений на множители (в частности, полугруппы $\mathscr{R}_{m}$, рассмотренные во введении) являются основными (для наших целей) примерами полугрупп над группами.

Пусть $\left\{g_{i}\right\}_{i \in I}$ - некоторое множество элементов группы $G$. Для каждого $i \in I$ обозначим через $O_{g_{i}} \subset G$ множество всех элементов из $G$, сопряженных элементу $g_{i}$ (орбиту элемента $g_{i}$ при действии группы $G$ на себе внутренними автоморфизмами). Назовем объединение $O=O_{\left\{g_{i}\right\}_{i \in I}}=\bigcup_{i \in I} O_{g_{i}} \subset G$ nолнъим множеством сопряженных элементов (т. е. элементов, сопряженных элементам из множества $\left.\left\{g_{i}\right\}_{i \in I}\right)$, а пару $(G, O)$ - оснащенной группой.

Сопоставим каждому полному множеству $O$ сопряженных элементов алфавит $X=X_{O}=\left\{x_{g} \mid g \in O\right\}$ и для каждой пары букв $x_{g_{1}}, x_{g_{2}} \in X, g_{1} \neq g_{2}$, обозначим через $R_{g_{1}, g_{2} ; 1}$ и $R_{g_{1}, g_{2} ; \text { r }}$ следующие соотношения: $R_{g_{1}, g_{2} ; 1}$ имеет вид

$$
x_{g_{1}} \cdot x_{g_{2}}=x_{g_{2}} \cdot x_{g_{2}^{-1} g_{1} g_{2}},
$$

если $g_{2} \neq \mathbf{1}$, и $x_{g_{1}} \cdot x_{\mathbf{1}}=x_{g_{1}}$, если $g_{2}=\mathbf{1}$, а $R_{g_{1}, g_{2} ; r}$ имеет вид

$$
x_{g_{1}} \cdot x_{g_{2}}=x_{g_{1} g_{2} g_{1}^{-1}} \cdot x_{g_{1}},
$$

если $g_{1} \neq \mathbf{1}$, и $x_{\mathbf{1}} \cdot x_{g_{2}}=x_{g_{2}}$, если $g_{1}=\mathbf{1}$.

Положив

$$
\mathscr{R}=\left\{R_{g_{1}, g_{2} ; \mathrm{r}}, R_{g_{1}, g_{2} ; \mathrm{l}} \mid\left(g_{1}, g_{2}\right) \in O \times O, g_{1} \neq g_{2}\right\},
$$

определим с помощью множества соотношений $\mathscr{R}$ полугруппу

$$
S(G, O)=\left\langle x_{g} \in X \mid R \in \mathscr{R}\right\rangle .
$$

Определим также гомоморфизм $\alpha: S(G, O) \rightarrow G$, заданный на порождающих элементах $x_{g}$ формулой $\alpha\left(x_{g}\right)=g$ для каждого $x_{g} \in X$, и назовем его гомоморфизмом произведения. Кроме того, определим действие $\lambda$ группы $G$ на множестве $X$, положив для каждого $g \in G$

$$
x_{a} \in X \mapsto \lambda(g)\left(x_{a}\right)=x_{g a g^{-1}} \in X .
$$

Легко видеть, что множество соотношений $\mathscr{R}$ инвариантно относительно этого действия и, следовательно, $\lambda$ определяет гомоморфизм $\lambda: G \rightarrow \operatorname{Aut}(S(G, O))$ (левое действие или действие сопряжением). Положим $\lambda_{S}=\lambda \circ \alpha$ и $\rho_{S}=\rho \circ \alpha$, где $\rho(g)=\lambda\left(g^{-1}\right)$ для $g \in G$. 
УтВеРЖДЕНИЕ 2.1 [20]. Для любых $s_{1}, s_{2} \in S(G, O)$ имеют место следующие равенства:

$$
s_{1} \cdot s_{2}=s_{2} \cdot \rho_{S}\left(s_{2}\right)\left(s_{1}\right)=\lambda_{S}\left(s_{1}\right)\left(s_{2}\right) \cdot s_{1} .
$$

Из утверждения 2.1 следует, что $(S(G, O), G, \alpha, \lambda)$ является полугруппой над $G$. Назовем такие полугруппы полугруппами разложений на множители элементов группы $G$ с множителями из $O$. Отметим, что $S:(G, O) \mapsto S(G, O)$ является функтором из категории оснащенных групп в категорию полугрупп. В частности, если $O_{1} \subset O_{2}$ - два полных множества сопряженных элементов в группе $G$, то тождественное отображение id $G \rightarrow G$ определяет вложение полугрупп $\operatorname{id}_{O_{1}, O_{2}}: S\left(G, O_{1}\right) \rightarrow S\left(G, O_{2}\right)$. Поэтому для каждой группы $G$ полугруппа $S(G, G)$ является универсальной полугруппой над $G$, т. е. каждая полугруппа $S(G, O)$ над $G$ канонически вкладывается в $S(G, G)$ с помощью $\operatorname{id}_{O, G}$. Если группа $G$ фиксирована, то полугруппа $S(G, O)$ будет обозначаться как $S_{O}$.

Число $\ln (s)=n$ называется длиной элемента $s=x_{g_{1}} \cdot \ldots \cdot x_{g_{n}} \in S_{O}$, если все $g_{i} \neq 1$.

Обозначим через $G_{O}$ подгруппу в $G$, порожденную образом гомоморфизма $\alpha: S_{O} \rightarrow G$, и для каждого $s \in S_{O}$ обозначим через $G_{s}$ подгруппу в $G$, порожденную образами $\alpha\left(x_{g_{1}}\right)=g_{1}, \ldots, \alpha\left(x_{g_{n}}\right)=g_{n}$ множителей $x_{g_{1}}, \ldots, x_{g_{n}}$ элемента $s=x_{g_{1}} \ldots x_{g_{n}}$.

УТВЕРЖДЕНИЕ 2.2. Подгруппа $G_{s}$ группы $G$ не зависит от представления элемента $s$ в виде слова от $x_{g_{i}}$ из алфавита $X_{O}$.

Доказательство следующего предложения является простым упражнением.

ПРЕДЛОЖЕНИЕ 2.1 [20]. Для любого полного множества сопряженных элементов $O$ и любого $s \in S_{O}$ имеем:

1) $\operatorname{ker} \lambda$ совпадает с иентрализатором $C_{O}$ группь $G_{O}$ в $G$;

2) если $\alpha(s)$ принадлежит центру группы $G_{s}$, то для каждого $g \in G_{s}$ действие $\lambda(\mathrm{g})$ оставляет неподвиюным элемент $s \in S_{O}$;

3) если $\alpha\left(s_{1} \cdot s_{2}\right)$ принадлежит центру группы $G_{s_{1} \cdot s_{2}}$, то $s_{1} \cdot s_{2}=s_{2} \cdot s_{1}$.

В качестве примера рассмотрим симметрическую группу $\mathscr{S}_{m}$, действующую на множестве $\{1, \ldots, m\}=[1, m]$. Очевидно, полугруппа $\mathscr{R}_{m}$ римановых поверхностей над диском (см. введение) и полугруппа $S_{\mathscr{S}_{m}}$ естественным образом изоморфны как полугруппы над группой $\mathscr{S}_{m}$. Из предложения $\left.2.1,3\right)$ следует, что подполугруппа $\mathscr{R}_{m, \mathbf{1}}$ является коммутативной.

Пусть $(i, j) \in \mathscr{S}_{m}$ - транспозиция, переставляющая элементы $i$ и $j$ из $[1, m]$. Обозначим через $T_{m}=O_{\{(1,2)\}}$ множество транспозиций в $\mathscr{S}_{m}$. Элемент

$h_{m, g}=\left(x_{(1,2)} \cdot x_{(1,2)}\right)^{g+1} \cdot\left(x_{(2,3)} \cdot x_{(2,3)}\right) \cdot \ldots \cdot\left(x_{(m-1, m)} \cdot x_{(m-1, m)}\right) \in S_{T_{m}} \subset S_{\mathscr{S}_{m}}$

называется элементом Гурвица рода g. Он обладает следующими замечательными свойствами.

ПреДЛОЖЕНИЕ 2.2. Элемент Гурвица $h_{m, g}$ принадлежит центру полугруппы $S_{\mathscr{S}_{m}}$ и является неподвижным элементом при действии сопряжением группы $\mathscr{S}_{m}$ на полугруппе $S_{\mathscr{S}_{m}}$. 
ДокАЗАТЕЛЬство следует из предложения 2.1 , так как $\alpha\left(h_{m, g}\right)=1$ и элементы $(i, i+1), i=1, \ldots, m-1$, с одной стороны, порождают группу $\left(\mathscr{S}_{m}\right)_{h_{m, g}}$, а с другой стороны, порождают всю симметрическую группу $\mathscr{S}_{m}$.

Более того, элемент Гурвица $h_{m, g}$ в полугруппе $S_{T_{m}}$ однозначно определяется следующими тремя условиями.

ТЕОРЕмА ГурвицА. Пусть элемент $s \in S_{T_{m}}$ удовлетворяет условиям:

(i) $\left(\mathscr{S}_{m}\right)_{s}=\mathscr{S}_{m}$;

(ii) длина $\ln (s)=2(g+m)$;

(iii) $\alpha(s)=1$.

Тогда $s=h_{m, g}$.

Приведенная выше теорема Гурвица имеет достаточно простое алгебраическое доказательство, а с другой стороны, по существу является переформулировкой в алгебраических терминах упоминавшейся во введении теоремы Гурвица о связности пространства общих накрытий степени $m$ проективной прямой алгебраическими кривыми рода $g$.

2.2. Полугруппы разложений на множители с частичным сокращением. Пусть $O_{1}=O_{\left\{g_{i}\right\}_{i \in I}}$ и $O_{2}=O_{\left\{g_{i}\right\}_{i \in J}}$ - два полных множества сопряженных элементов в группе $G$ такие, что $O_{1} \cap O_{2}=\varnothing$ и если $g \in O_{2}$, то $g^{-1}$ также принадлежит множеству $\mathrm{O}_{2}$, и пусть $O=\mathrm{O}_{1} \cup \mathrm{O}_{2}$.

Рассмотрим полугруппу (с единицей)

$$
\bar{S}_{O, O_{2}}=\left\langle x_{g} \in X_{O} \mid R \in \mathscr{R} \cup \overline{\mathscr{R}}\right\rangle,
$$

где $\mathscr{R}$ - множество соотношений, определяющих полугруппу $S_{O}$, и

$$
\overline{\mathscr{R}}=\left\{x_{g} \cdot x_{g^{-1}}=x_{g^{-1}} \cdot x_{g}=\mathbf{1} \mid g \in O_{2}\right\}
$$

- множество соотношений сокращения. Существует естественный гомоморфизм полугрупп

$$
c: S_{O} \rightarrow \bar{S}_{O, O_{2}} .
$$

Легко видеть, что $\bar{S}_{O, O_{2}}$ можно рассматривать как полугруппу над группой $G$ с естественным гомоморфизмом произведения $\bar{\alpha}: \bar{S}_{O, O_{2}} \rightarrow G$ таким, что $\alpha=$ $\bar{\alpha} \circ c$.

Отметим, что $S_{O, O_{2}}$ является группой тогда и только тогда, когда множество $O_{1}$ либо пусто, либо состоит из одного элемента $g=\mathbf{1}$.

2.3. Эквивалентность Гурвица. Пусть, как и выше, $O \subset G$ - полное множество сопряженных элементов в группе $G$. Упорядоченное множество

$$
\left\{g_{1}, \ldots, g_{n} \mid g_{i} \in O\right\}, \quad n \in \mathbb{Z},
$$

называется разложением на множители элемента $g=g_{1} \ldots g_{n} \in G$ с множителями из $O$. Обозначим $F_{O}=\bigcup_{n} O^{n}$ множество всех возможных разложений на множители элементов из группы $G$ с множителями из $O$. Перестановка множителей в произведении $g_{1} \ldots g_{n}=g$, не меняющая это произведение, приводит к следующему определению. Преобразование, которое заменяет 
в $\left\{g_{1}, \ldots, g_{n}\right\}$ некоторые два соседних множителя $\left(g_{i}, g_{i+1}\right)$ на $\left(g_{i+1}, g_{i+1}^{-1} g_{i} g_{i+1}\right)$ или $\left(g_{i} g_{i+1} g_{i}^{-1}, g_{i}\right)$ и сохраняет все остальные множители, называется преобразованием Гурвица. Два разложения на множители называются Гурвиц-эквивалентными, если одно из них может быть получено из другого с помощью конечного числа преобразований Гурвица. Отметим, что Гурвиц-эквивалентные разложения на множители являются разложениями на множители одного и того же элемента.

Существует естественное отображение $\varphi: F_{O} \rightarrow S_{O}$, заданное формулой

$$
\varphi\left(\left\{g_{1}, \ldots, g_{n}\right\}\right)=x_{g_{1}} \cdot \ldots \cdot x_{g_{n}} .
$$

Очевидно, имеет место следующее утверждение.

УТВЕРЖДЕНИЕ 2.3. Два разложения на множители $y=\left\{y_{1}, \ldots, y_{n}\right\} u z=$ $\left\{z_{1}, \ldots, z_{n}\right\}$ Гурвиц-эквивалентны тогда и только тогда, когда $\varphi(y)=\varphi(z)$.

Ниже, в согласии с утверждением 2.3, классы Гурвиц-эквивалентных разложений на множители с множителями из $O$ будут отождествляться с их образами в $S_{O}$. Кроме того, для упрощения обозначений разложение на множители $s=x_{g_{1}} \cdot \ldots \cdot x_{g_{n}} \in S_{O}$ элемента $g=g_{1} \ldots g_{n} \in G$ будет записываться в виде $s=g_{1} \cdot \ldots \cdot g_{n}$ (другими словами, через $g_{1} g_{2}$ будем обозначать произведение элементов $g_{1}$ и $g_{2}$ в группе $G$, а через $g_{1} \cdot g_{2}$ - произведение элементов $x_{g_{1}}$ и $x_{g_{2}}$ в полугруппе $\left.S_{O}\right)$.

2.4. Регенерация разложений на множители. Рассмотрим два полных множества $O_{1}, O_{2}$ сопряженных элементов в группе $G$ и ассоциированные с ними полугруппы $S_{O_{1}}$ и $S_{O_{2}}$. Отображение $\psi: X_{2} \rightarrow S_{O_{1}}$ может быть доопределено до гомоморфизма $\psi: S_{O_{2}} \rightarrow S_{O_{1}}$ тогда и только тогда, когда для любых $x_{g_{i}}, x_{g_{j}} \in X_{2}$ равенства

$$
\psi\left(x_{g_{i}}\right) \cdot \psi\left(x_{g_{j}}\right)=\psi\left(x_{g_{j}}\right) \cdot \psi\left(x_{g_{j}^{-1} g_{i} g_{j}}\right)
$$

и

$$
\psi\left(x_{g_{i}}\right) \cdot \psi\left(x_{g_{j}}\right)=\psi\left(x_{g_{i} g_{j} g_{i}^{-1}}\right) \cdot \psi\left(x_{g_{i}}\right)
$$

имеют место в $S_{O_{1}}$.

Мы скажем, что гомоморфизм $\psi$ определен над $G$, если $\alpha\left(x_{g}\right)=\alpha\left(\psi\left(x_{g}\right)\right)$ для всех $g \in \mathrm{O}_{2}$.

Пример 2.1. Пусть $O_{1}-$ полное множество сопряженных элементу $g_{1} \in G$ и $\mathrm{O}_{2}$ - полное множество сопряженных элементу $g_{1}^{2}$. Предположим, что отображение $\phi: O_{1} \rightarrow O_{2}$, заданное формулой $\phi(g)=g^{2}$ для $g \in O_{1}$, является взаимно однозначным. Тогда отображение $\psi: X_{2} \rightarrow S_{O_{1}}$, заданное формулой $\psi\left(x_{g}\right)=x_{\phi^{-1}(g)} \cdot x_{\phi^{-1}(g)}$, определяет гомоморфизм $\psi: S_{O_{2}} \rightarrow S_{O_{1}}$ над $G$.

Пример 2.1 может быть обобщен следующим образом.

ПримеР 2.2. Выберем $n$-множество $\left\{g_{1}, \ldots, g_{n}\right\}$ элементов в полном множестве сопряженных элементов $O_{1} \subset G$ и $k$-множество произведений

$$
s_{j}\left(x_{g_{1}}, \ldots, x_{g_{n}}\right)=x_{g_{i_{1}(j)}} \cdot \ldots \cdot x_{g_{i_{m(j)}}(j)} \in S_{O_{1}}, \quad \ln \left(s_{j}\right)=m(j), \quad j=1, \ldots, k .
$$


Рассмотрим полное множество сопряженных элементов $O_{2}=O_{\bar{s}_{1}} \cup \cdots \cup O_{\bar{s}_{k}}$, где $O_{\bar{s}_{j}}-$ полное множество элементов, сопряженных элементам $\bar{s}_{j}=\alpha\left(s_{j}\right) \in G$. Предположим, что $\bar{s}_{i}$ и $\bar{s}_{j}$ не сопряжены в $G$ для $i \neq j$. Тогда отображение $X_{2} \rightarrow S_{O_{1}}$, заданное $x_{g \bar{s}_{j} g^{-1}} \mapsto \lambda(g)\left(s_{j}\right) \in S_{O_{1}}$, может быть однозначно продолжено до гомоморфизма $r: S_{O_{2}} \rightarrow S_{O_{1}}$, определенного над $G$. Гомоморфизм $r$ называется регенерачией множества элементов $\left\{s_{j}\right\}$.

Ниже мы будем использовать некоторое обобщение этого понятия (которое уже не будет гомоморфизмом), определяемое следующим образом. В обозначениях примера 2.2 положим $O=O_{1} \cup O_{2}$ и рассмотрим элемент $z=z_{1} \cdot z_{2} \in S_{O}$, где $z_{1} \in S_{O_{2}}, z_{2} \in S_{O}$. Элемент

$$
\bar{z}=r\left(z_{1}\right) \cdot z_{2} \in S_{O}
$$

называется частичной регенерацией элемента $z$.

\section{§ 3. Полугруппы над группами кос}

В этом параграфе группа $G=\mathrm{Br}_{m}$ - это группа кос с $m$ нитями, введенная Э. Артином в 1925 г. в работе [21].

3.1. Реализации группы кос. Как абстрактная группа, группа кос $\mathrm{Br}_{m}$ имеет следующее копредставление. Она порождается множеством элементов $\left\{a_{1}, \ldots, a_{m-1}\right\}$, связанных соотношениями

$$
\begin{aligned}
a_{i} a_{i+1} a_{i} & =a_{i+1} a_{i} a_{i+1}, & & 1 \leqslant i \leqslant m-1, \\
a_{i} a_{k} & =a_{k} a_{i}, & & |i-k| \geqslant 2
\end{aligned}
$$

(такие порождающие группы кос $\mathrm{Br}_{m}$ будем называть стандартными).

Напомним, что группа кос $\mathrm{Br}_{m}$ имеет несколько естественных реализаций. Первая из них - это группа геометрических кос, элементами которой являются наборы $b$, состоящие из $m$ попарно непересекающихся путей

$$
\gamma_{j}(t)=\left\{\left(x_{j}(t), y_{j}(t), t\right)\right\} \subset \mathbb{R}^{3}=\{(x, y, z) \mid x, y, z \in \mathbb{R}\},
$$

$t \in[0,1], j=1, \ldots, m$ (рассматриваемых с точностью до непрерывной изотопии), начала и концы которых фиксированы (пусть для определенности $\left(x_{j}(0)\right.$, $\left.\left.y_{j}(0)\right)=\left(x_{j}(1), y_{j}(1)\right)=(-j, 0) \in \mathbb{R}^{2}\right)$. Произведение кос

$$
b_{1}=\left\{\left(\gamma_{1, j, 1}(t), \gamma_{1, j, 2}(t), t\right)\right\} \quad \text { и } b_{2}=\left\{\left(\gamma_{2, j, 1}(t), \gamma_{2, j, 2}(t), t\right)\right\}
$$

- это коса $b=b_{1} b_{2}=\left\{\left(\gamma_{j, 1}(t), \gamma_{j, 2}(t), t\right)\right\}$, где

$$
\gamma_{j, l}(t)=\left\{\begin{array}{ll}
\gamma_{1, j, l}(2 t), & \text { если } 0 \leqslant t \leqslant \frac{1}{2}, \\
\gamma_{2, j, l}(2 t-1), & \text { если } \frac{1}{2} \leqslant t \leqslant 1,
\end{array} \quad l=1,2,\right.
$$

а порождающий элемент $a_{j}$ - это коса, изображенная на рис. 1 .

Вторая реализация группы $\mathrm{Br}_{m}$ - это факторгруппа $\mathrm{Br}_{m}[D, K]$ группы диффеоморфизмов проколотого диска $\bar{D} \backslash K$ с $m$ проколами $K=\left\{p_{1}, \ldots, p_{m}\right\} \subset$ $\bar{D} \backslash \partial \bar{D}$, тождественных на границе $\partial \bar{D}$ диска $\bar{D}=\{u \in \mathbb{C}|| u \mid \leqslant R\}$, по модулю 


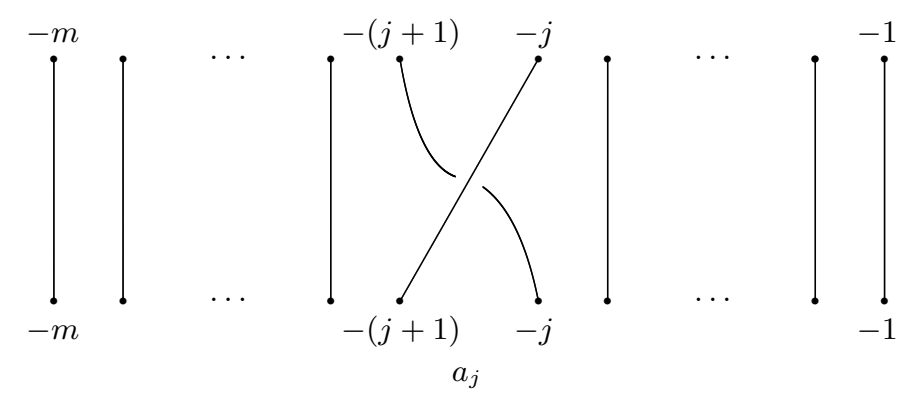

Рис. 1

диффеоморфизмов, гомотопных тождественному диффеоморфизму. (Для определенности будем предполагать, что группа $\operatorname{Br}_{m}[D, K]$ действует справа, т. е. $g f=f \circ g$, где о означает композицию диффеоморфизмов: сначала применяем диффеоморфизм $g$, а затем $f$ ). Чтобы задать изоморфизм группы $\operatorname{Br}_{m}[D, K]$ и абстрактной группы кос $\mathrm{Br}_{m}$, порожденной стандартными порождающими $a_{1}, \ldots, a_{m-1}$, выберем $m-1$ гладких путей $l_{j}, j=1, \ldots, m-1$, с началами в точках $p_{j}$ и концами в точках $p_{j+1}$ таких, что путь $l=l_{1} \cup \cdots \cup l_{m-1}$ является простым путем без самопересечений. Тогда группа $\operatorname{Br}_{m}[D, K]$ порождается полуповоротами, ассоциированными с путями $l_{j}$ (обозначим их снова через $a_{j}$, $j=1, \ldots, m-1$, так как сопоставление этих полуповоротов соответствующим образующим группы $\mathrm{Br}_{m}$ задает изоморфизм групп $\mathrm{Br}_{m}$ и $\left.\mathrm{Br}_{m}[D, K]\right)$. Чтобы определить полуповорот $a_{j}$, выберем некоторую достаточно маленькую окрестность $U$ пути $l_{j}, K \cap U=\left\{p_{j}, p_{j+1}\right\}$, а также сохраняющее ориентацию гладкое вложение $\psi: \bar{D} \longrightarrow \mathbb{C}^{1}$ ( $\mathbb{C}^{1}$ взято с обычной “комплексной” ориентацией) так, что $\psi\left(l_{j}\right)=[-1,1]=\left\{z \in \mathbb{C}^{1} \mid \operatorname{Re} z \in[-1,1], \operatorname{Im} z=0\right\}, \psi\left(p_{i}\right)=1, \psi\left(p_{i+1}\right)=-1$ и $\psi(U)=\left\{z \in \mathbb{C}^{1}|| z \mid<2\right\}$. Пусть $\alpha(r), r \geqslant 0$, - гладкая монотонная вещественная функция такая, что $\alpha(r)=1$ для $r \in[0,3 / 2]$ и $\alpha(r)=0$ для $r \geqslant 2$. Рассмотрим диффеоморфизм $h: \mathbb{C}^{1} \rightarrow \mathbb{C}^{1}$, действующий на $z \in \mathbb{C}^{1}, z=r e^{i \varphi}$, по правилу $h(z)=r e^{i(\varphi+\alpha(r) \pi)}$. Очевидно, что ограничение диффеоморфизма $h$ на диск $\left\{z \in \mathbb{C}^{1}|| z \mid \leqslant 3 / 2\right\}$ совпадает с поворотом на угол $\pi$, а ограничение на $\left\{z \in \mathbb{C}^{1}|| z \mid \geqslant 2\right\}$ является тождественным диффеоморфизмом. Диффеоморфизм $a_{j}=\psi^{-1} \circ h \circ \psi$ называется полуповоротом, ассоциированным с путем $l_{j}$.

Третья реализация группы $\mathrm{Br}_{m}$ - это подгруппа $B_{m}$ группы автоморфизмов Aut $\mathbb{F}_{m}$ свободной группы $\mathbb{F}_{m}$, свободно порожденной элементами $x_{1}, \ldots, x_{m}$, такая, что автоморфизм $b \in \operatorname{Aut} \mathbb{F}_{m}$ принадлежит группе $B_{m}$ тогда и только тогда, когда

(i) существует перестановка $\sigma_{b} \in \mathscr{S}_{m}$ такая, что для $i=1, \ldots, m$ образ $\left(x_{i}\right) b$ сопряжен элементу $x_{\sigma_{b}(i)}$;

(ii) $x_{1} \ldots x_{m}=\left(x_{1} \ldots x_{m}\right) b$.

Можно показать, что автоморфизмы $a_{i} \in B_{m}$, заданные формулами

$$
\begin{aligned}
\left(x_{j}\right) a_{i} & =x_{j} \quad \text { при } j \neq i, i+1, \\
\left(x_{i}\right) a_{i} & =x_{i} x_{i+1} x_{i}^{-1}, \\
\left(x_{i+1}\right) a_{i} & =x_{i},
\end{aligned}
$$


порождают группу $B_{m}$, удовлетворяют соотношениям (3) и гомоморфизм из группы $\mathrm{Br}_{m}$ в группу $B_{m}$, переводящий порождающие элементы $a_{i} \in \mathrm{Br}_{m}$ в автоморфизмы $a_{i} \in B_{m} \subset \mathrm{Aut} \mathbb{F}_{m}$, является изоморфизмом и тем самым определяет так называемое стандартное действие группы кос $\mathrm{Br}_{m}$ на свободной группе $\mathbb{F}_{m}=\left\langle x_{1}, \ldots, x_{m}\right\rangle$. Геометрически это действие может быть реализовано как действие группы $\mathrm{Br}_{m}[D, K]$ на фундаментальной группе $\pi\left(\bar{D} \backslash K, p_{0}\right)$, $p_{0} \in \partial \bar{D}$.

3.2. Стабильная эквивалентность. Обозначим через $\mathrm{Br}_{m}^{+}$полугруппу, порожденную как абстрактная полугруппа элементами $\left\{a_{1}, \ldots, a_{m-1}\right\}$, связанными соотношениями (3).

ТЕОРЕМА ГАРСАЙДА [22]. Естественный гомоморфизм $i: \mathrm{Br}_{m}^{+} \rightarrow \mathrm{Br}_{m}$ является вложением.

Исходя из этой теоремы, полугруппа $\mathrm{Br}_{m}^{+}$отождествляется с ее образом $i\left(\mathrm{Br}_{m}^{+}\right)$в $\mathrm{Br}_{m}$ и образы $i(g)$ элементов $g \in \mathrm{Br}_{m}^{+}$называются положительнъми элементами группы $\mathrm{Br}_{m}$.

Обозначим через $A_{k}=A_{k}(m), k \in \mathbb{Z}$, полное множество сопряженных элементу $a_{1}^{k+1}$ в группе $\mathrm{Br}_{m}$ (напомним, что все порождающие $a_{1}, \ldots, a_{m-1}$ сопряжены друг другу). В $\S 5$ и $\S 6$, в которых пойдет речь о $H$-изотопии и регулярной гомотопии каспидальных кривых Гурвица, нам понадобятся полугруппы $\mathscr{A}=S_{A}$ и $\overline{\mathscr{A}}=S_{\bar{A}}$, где $A=\bigcup_{k \geqslant 0} A_{k}$ и $\bar{A}=A \cup A_{-3}$, являющиеся полугруппами разложений на множители брэйд-монодромии (см. $\S 4)$ каспидальных (соответственно каспидальных с отрицательными ноудами) кривых Гурвица.

Рассмотрим вначале полугруппу $\mathscr{A}_{0}=S_{A_{0}}$ как подполугруппу универсальной полугруппы $S_{\mathrm{Br}_{m}}$ над группой $\mathrm{Br}_{m}$. Слово $g=a_{i_{1}} \ldots a_{i_{n}}$ в алфавите $\left\{a_{1}, \ldots, a_{m-1}\right\}$ определяет элемент $\bar{g}\left(a_{1}, \ldots, a_{m-1}\right)=a_{i_{1}} \cdot \ldots \cdot a_{i_{n}} \in S_{A_{0}}$. С другой стороны, $g$ определяет элемент $\tilde{g}=a_{i_{1}} \ldots a_{i_{n}}$ в $\mathrm{Br}_{m}^{+}$.

ЛЕмма 3.1 [23]. Отображение $\nu: \mathrm{Br}_{m}^{+} \rightarrow \mathscr{A}_{0}$, заданное равенством $\nu(\tilde{g})=\bar{g}$, является инбективным гомоморфизмом полугрупп.

ДокАЗАТЕЛЬСтво. Чтобы показать, что $\nu$ является гомоморфизмом, достаточно проверить, что соотношения (3) имеют место в $\mathscr{A}_{0}$. Имеем

$$
\begin{aligned}
a_{i} \cdot a_{i+1} \cdot a_{i} & =a_{i+1} \cdot\left(a_{i+1}^{-1} a_{i} a_{i+1}\right) \cdot a_{i}=a_{i+1} \cdot a_{i} \cdot\left(a_{i}^{-1} a_{i+1}^{-1} a_{i} a_{i+1} a_{i}\right) \\
& =a_{i+1} \cdot a_{i} \cdot\left(a_{i}^{-1} a_{i+1}^{-1} a_{i+1} a_{i} a_{i+1}\right)=a_{i+1} \cdot a_{i} \cdot a_{i+1}
\end{aligned}
$$

для $1 \leqslant i \leqslant m-1$ и

$$
a_{i} \cdot a_{k}=a_{k} \cdot\left(a_{k}^{-1} a_{i} a_{k}\right)=a_{k} \cdot a_{i}
$$

для $|i-k| \geqslant 2$. Гомоморфизм $\nu$ инъективен, так как согласно теореме Гарсайда $\alpha \circ \nu$ является тождественным изоморфизмом. Лемма 3.1 доказана.

Пусть $\Delta_{m}$ - так называемый элемент Гарсайда:

$$
\Delta_{m}=\left(a_{1} \ldots a_{m-1}\right) \ldots\left(a_{1} a_{2} a_{3}\right)\left(a_{1} a_{2}\right) a_{1} .
$$

Как хорошо известно, элемент

$$
\Delta_{m}^{2}=\left(a_{1} \ldots a_{m-1}\right)^{m}
$$


порождает центр группы $\mathrm{Br}_{m}$. Отметим, что определение элемента Гарсайда зависит от выбора стандартных порождающих $a_{1}, \ldots, a_{m-1}$, тогда как элемент $\Delta_{m}^{2}$ не зависит от этого выбора. Элемент $\Delta_{m}^{2}$ называется полным поворотом (в реализации группы кос $\mathrm{Br}_{m}$ как группы диффеоморфизмов проколотого диска $\bar{D} \backslash K$, где $\bar{D}=\{u \in \mathbb{C}|| u \mid \leqslant 2\}$ и $K \subset D_{1}=\{u \in \mathbb{C}|| u \mid<1\}$, элемент $\Delta_{m}^{2}$ - это диффеоморфизм $h: \bar{D} \rightarrow \bar{D}$, действующий на $u \in \bar{D}, u=r e^{i \varphi}$, по правилу $h(u)=r e^{i(\varphi+2 \alpha(r) \pi)}$, где $\alpha$ - гладкая монотонная вещественная функция такая, что $\alpha(r)=1$ для $r \in[0,1]$ и $\alpha(r)=0$ для $r=2$; т. е. ограничение диффеоморфизма $h$ на диск $D_{1}$ совпадает с поворотом на угол $\left.2 \pi\right)$.

Обозначим через $\delta_{m}^{2}$ элемент в $\mathscr{A}_{0} \subset S_{\mathrm{Br}_{m}}$, равный

$$
\delta_{m}^{2}=\left(a_{1} \cdot \ldots \cdot a_{m-1}\right)^{m} .
$$

ЛЕмма 3.2. Элемент $\delta_{m}^{2}$ неподвижен при действии сопряжением группъц $\mathrm{Br}_{m}$ на $S_{\mathrm{Br}_{m}}$, m.е. $\lambda(g)\left(\delta_{m}^{2}\right)=\delta_{m}^{2}$ для любого $g \in \mathrm{Br}_{m}$.

ДокАЗАТЕЛЬСтво следует из равенства $\alpha\left(\delta_{m}^{2}\right)=\Delta_{m}^{2}$ и предложения 2.1, примененного к $s=\delta_{m}^{2}$ (для которого $\left.\left(\mathrm{Br}_{m}\right)_{s}=\mathrm{Br}_{m}\right)$.

Скажем, что элемент $s_{1} \in S_{\mathrm{Br}_{m}}$ стабилъно равен элементу $s_{2} \in S_{\mathrm{Br}_{m}}$, если найдется такое целое число $n \geqslant 1$, что

$$
s_{1} \cdot\left(\delta_{m}^{2}\right)^{n}=s_{2} \cdot\left(\delta_{m}^{2}\right)^{n} .
$$

Теорема 3.1 [20]. Пусть $O_{b_{1}}, \ldots, O_{b_{n}}$ - орбиты элементов $b_{1}, \ldots, b_{n}$ при действии сопряжением группь $\mathrm{Br}_{m}, u$ пусть $\sigma$ - некоторая перестановка из $\mathscr{S}_{n}$. Тогда для любых $\bar{b}_{i} \in O_{b_{i}}, 1 \leqslant i \leqslant n$, элементы $s_{1}=b_{1} \cdot \ldots \cdot b_{n} u s_{2}=$ $\bar{b}_{\sigma(1)} \cdot \ldots \bar{b}_{\sigma(n)}$ стабильно равны тогда и только тогда, когда $\alpha\left(s_{1}\right)=\alpha\left(s_{2}\right)$.

Доказательство этой теоремы базируется на теореме Гарсайда и следующей лемме.

ЛЕмма 3.3 [22]. Для любого $g \in \mathrm{Br}_{m}$ найдутся положителъные элементы $r_{1}, r_{2} \in \mathrm{Br}_{m}$ и целые числа $k, p \in \mathbb{Z}, p \geqslant 1$, такие, что

1) $g=\Delta_{m}^{2 k} r_{1}$

2) $g r_{2}=\Delta_{m}^{2 p}$.

ДокАЗАТЕЛЬСтво следует из теоремы 5 в [22].

Приведем набросок доказательства теоремы 3.1. Переставив множители в $s_{2}$ и применив лемму 3.3 , можем считать, что $s_{2}=\bar{b}_{1} \cdot \ldots \cdot \bar{b}_{n}$, где $\bar{b}_{i}=r_{i} b_{i} r_{i}^{-1}$ для всех $i$, причем все $r_{i}$ являются положительными элементами. Из предложения $2.1,3)$ следует (после циклической перестановки множителей), что $\delta_{m}^{2}$ может быть записан в виде $\delta_{m}^{2}=a_{k} \cdot \delta_{k}^{\prime}$, где $\delta_{k}^{\prime} \in \mathscr{A}_{0}$, и, кроме того, в произведении $s_{2} \cdot\left(\delta_{m}^{2}\right)^{M}=\bar{b}_{1} \cdot \ldots \cdot \bar{b}_{n} \cdot\left(\delta_{m}^{2}\right)^{M}$ мы можем передвинуть каждый множитель $\delta_{m}^{2}$ влево, не меняя остальных множителей. Следовательно, если $r_{i}=r_{i}^{\prime} a_{k}$, то

$$
\bar{b}_{i} \cdot \delta_{m}^{2}=\left(a_{k} r_{i}^{\prime} b_{i} r_{i}^{\prime-1} a_{k}^{-1}\right) \cdot a_{k} \cdot \delta_{k}^{\prime}=a_{k} \cdot\left(r_{i}^{\prime} b_{i} r_{i}^{\prime-1}\right) \cdot \delta_{k}^{\prime} .
$$

Поэтому, если $M$ - достаточно большое число, то $s_{2} \cdot\left(\delta_{m}^{2}\right)^{M}=b_{1} \cdot \ldots \cdot b_{n} \cdot s_{2}^{\prime}$, где $s_{2}^{\prime} \in \mathscr{A}_{0}$. Записав каждый множитель $g_{i}$, входящий в $s_{2}^{\prime}$, в виде $g_{i}=r_{i} a_{1} r_{i}^{-1}$ и 
повторив приведенные выше рассуждения, получаем, что элемент $s_{2} \cdot\left(\delta_{m}^{2}\right)^{M+M_{1}}$ при достаточно большом $M_{1}$ может быть записан в виде $s_{2} \cdot\left(\delta_{m}^{2}\right)^{M+M_{1}}=$ $b_{1} \cdot \ldots \cdot b_{n} \cdot s_{2}^{\prime \prime}$, где все множители, входящие в разложение на множители элемента $s_{2}^{\prime \prime},-$ это порождающие элементы $a_{i}$. Имеем

$$
\alpha\left(s_{2} \cdot\left(\delta_{m}^{2}\right)^{M+M_{1}}\right)=\alpha\left(s_{2}\right) \Delta_{m}^{2\left(M+M_{1}\right)}=\alpha\left(s_{1}\right) \alpha\left(s_{2}^{\prime \prime}\right) .
$$

Следовательно, $\alpha\left(s_{2}^{\prime \prime}\right)=\Delta_{m}^{2\left(M+M_{1}\right)}$ и согласно теореме Гарсайда и лемме 3.1 имеем $s_{2}^{\prime \prime}=\left(\delta_{m}^{2}\right)^{M+M_{1}}$, т. е. $s_{1} \cdot\left(\delta_{m}^{2}\right)^{M+M_{1}}=s_{2} \cdot\left(\delta_{m}^{2}\right)^{M+M_{1}}$.

3.3. Формула разложения на множители полного поворота с удвоенным числом нитей. Основной интерес для приложений к геометрии представляют разложения на множители полного поворота. Приведенная ниже формула разложения на множители полного поворота с удвоенным числом нитей позволяет строить много новых разложений на множители элемента $\Delta_{2 m}^{2}$ по известному разложению на множители элемента $\Delta_{m}^{2}$.

Пусть $a_{1}, \ldots, a_{2 m-1}$ - стандартные порождающие группы кос $\mathrm{Br}_{2 m}$. Для каждого $k \leqslant 2 m$ отождествим группу $\mathrm{Br}_{k}$ с подгруппой группы $\mathrm{Br}_{2 m}$, порожденной элементами $a_{1}, \ldots, a_{k-1}$. Кроме того, для каждой пары целых чисел $k \geqslant 1$ и $i \geqslant 0, k+i \leqslant 2 m$, обозначим через $\mathrm{Br}_{k, i}$ подгруппу группы $\mathrm{Br}_{2 m}$, порожденную элементами $a_{1+i}, \ldots, a_{k+i-1}$, и пусть $\Delta_{k, i}\left(\right.$ соответственно $\left.\Delta_{k, i}^{2}\right)$ это элемент Гарсайда (соответственно полный поворот) группы $\mathrm{Br}_{k, i}$.

Положим $a_{m, i}=a_{i} a_{2 m-i}$,

$$
\bar{c}_{m, i}=\left(a_{m, m-1} \ldots a_{m, i+1} a_{m, i}\right)^{-1} a_{m}\left(a_{m, m-1} \ldots a_{m, i+1} a_{m, i}\right), \quad 1 \leqslant i \leqslant m-1,
$$

$\bar{c}_{m, m}=a_{m}$ и

$$
c_{m, i}=\Delta_{m, m} \bar{c}_{m, i} \Delta_{m, m}^{-1} .
$$

Элемент $c_{m, i}$ является полуповоротом, ассоциированным с путем $l_{m, i}$, изображенным на рис. 2.

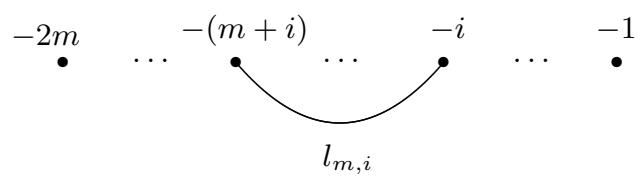

Рис. 2

Положим также

$$
\bar{r}_{m}=\bar{c}_{m, 1} \ldots \bar{c}_{m, m-1} \bar{c}_{m, m}
$$

и

$$
r_{m}=c_{m, 1} \ldots c_{m, m-1} c_{m, m}
$$

где $\bar{c}_{m, i}$ и $c_{m, i}$ определены формулами (5) и (6).

Лемма 3.4 [24]. В группе кос $\mathrm{Br}_{2 m}$ имеет место следующее равенство:

$$
\Delta_{2 m}=\Delta_{m, 0}^{2} \Delta_{m, m}^{2} \bar{r}_{m}
$$


ДокАЗАТЕЛЬСтво. Индукцией по $m$ можно проверить, что элементы $\Delta_{2 m}$ и $\Delta_{m, 0}^{2} \Delta_{m, m}^{2} \bar{r}_{m}$ одинаково действуют на свободных порождающих $x_{1}, \ldots, x_{2 m}$ свободной группы $\mathbb{F}_{2 m}$ при стандартном действии группы кос $\operatorname{Br}_{2 m}$.

TeOPEмa 3.2 [24]. В группе кос $\mathrm{Br}_{2 m}$ имеет место следующее равенство:

$$
\Delta_{2 m}^{2}=\Delta_{m, 0}^{4} \Delta_{m, m}^{4} r_{m}^{2}
$$

ДокАЗАТЕЛЬство. Согласно лемме 3.4 имеем $\Delta_{2 m}=\Delta_{m, 0}^{2} \Delta_{m, m}^{2} \bar{r}_{m}$. Легко видеть, что $\left[\Delta_{m, 0}, \Delta_{m, m}\right]=\mathbf{1}$ и $\left[\Delta_{2 m}, \Delta_{m, 0}^{2} \Delta_{m, m}^{2}\right]=\mathbf{1}$. Следовательно, $\left[\bar{r}_{m, m}, \Delta_{m, 0}^{2} \Delta_{m, m}^{2}\right]=\mathbf{1}$ и $\Delta_{2 m}^{2}=\Delta_{m, 0}^{4} \Delta_{m, m}^{4} \bar{r}_{m}^{2}$. Таким образом,

$$
\Delta_{2 m}^{2}=\Delta_{m, m}^{-1} \Delta_{2 m}^{2} \Delta_{m, m}=\Delta_{m, 0}^{4} \Delta_{m, m}^{4} r_{m}^{2}
$$

Гомоморфизм sh $=\mathrm{sh}_{m}: \mathrm{Br}_{m}=\mathrm{Br}_{m, 0} \rightarrow \mathrm{Br}_{2 m}$, заданный равенствами $\operatorname{sh}\left(a_{i}\right)=a_{m+i}$ для $i=1, \ldots, m-1$, называется гомоморфизмом сдвига.

Вложение $\mathrm{Br}_{m}=\mathrm{Br}_{m, 0} \subset \mathrm{Br}_{2 m}$ индуцирует вложение $S_{\mathrm{Br}_{m}} \subset S_{\mathrm{Br}_{2 m}}$. Гомоморфизм sh: $\mathrm{Br}_{m} \rightarrow \mathrm{Br}_{2 m}$ также индуцирует некоторое вложение $\mathrm{sh}: S_{\mathrm{Br}_{m}} \rightarrow$ $S_{\mathrm{Br}_{2 m}}$. Положим

$$
\tilde{r}_{m}=c_{m, 1} \cdot \ldots \cdot c_{m, m} \in S_{\mathrm{Br}_{2 m}}
$$

где $c_{m, i}$ определены формулой (6). Применяя формулу (8), получаем следующую формулу разложения на множители полного поворота с удвоенным числом нитей. Для четырех элементов $s_{1}, \ldots, s_{4} \in S_{\mathrm{Br}_{m}}$ таких, что $\alpha\left(s_{i}\right)=\Delta_{m}^{2}$, элемент

$$
\bar{s}=d\left(s_{1}, s_{2}, s_{3}, s_{4}\right)=s_{1} \cdot s_{2} \cdot \operatorname{sh}\left(s_{3}\right) \cdot \operatorname{sh}\left(s_{4}\right) \cdot \tilde{r}_{m} \cdot \tilde{r}_{m}
$$

является разложением на множители элемента $\Delta_{2 m}^{2}$ в $S_{\mathrm{Br}_{2 m}}$, т. е. $\alpha(\bar{s})=\Delta_{2 m}^{2}$.

Приведенная выше формула разложения на множители полного поворота с удвоенным числом нитей позволяет строить много новых разложений на множители элемента $\Delta_{2 m}^{2}$ по известному разложению на множители $s$ элемента $\Delta_{m}^{2}$, взяв, например, в качестве $s_{i}$ элементы $\lambda\left(g_{i}\right) s, g_{i} \in \mathrm{Br}_{m}$.

3.4. Группы, ассоциированные с элементами $s \in S_{\mathrm{Br}_{m}}$. Каждой подгруппе $B$ группы кос $\mathrm{Br}_{m}$ можно сопоставить группу $G_{B}$, заданную копредставлением

$$
G_{B}=\left\langle x_{1}, \ldots, x_{m} \mid x_{i}=\left(x_{i}\right) b, 1 \leqslant i \leqslant m, b \in B\right\rangle,
$$

где $\left(x_{i}\right) b$ - образ элемента $x_{i}$ при стандартном действии элемента $b \in \mathrm{Br}_{m}$ на свободной группе $\mathbb{F}_{m}=\left\langle x_{1}, \ldots, x_{m}\right\rangle$. Если $B$ является конечно порожденной группой, то группа $G_{B}$ является конечно определенной, и, более того, если группа $B$ порождена элементами $b_{1}, \ldots, b_{n}$, то (см. утверждение 1.6 в [24]) группа $G_{B}$ канонически изоморфна группе, заданной копредставлением

$$
\left\langle x_{1}, \ldots, x_{m} \mid x_{i}=\left(x_{i}\right) b_{j}, 1 \leqslant i \leqslant m, 1 \leqslant j \leqslant n\right\rangle .
$$

В частности, группа

$$
G(s)=G_{\left(\mathrm{Br}_{m}\right)_{s}}=\left\langle x_{1}, \ldots, x_{m} \mid x_{i}=\left(x_{i}\right) b_{j}, 1 \leqslant i \leqslant m, 1 \leqslant j \leqslant n\right\rangle,
$$


где группа $\left(\mathrm{Br}_{m}\right)_{s}$ порождена образами множителей элемента $s=b_{1} \cdot \ldots \cdot b_{n} \in$ $S_{\mathrm{Br}_{m}}$ в группе $\mathrm{Br}_{m}$ при гомоморфизме произведения (см. п. 2.1), не зависит от разложения элемента $s$ в произведение порождающих элементов полугруппы $S_{\mathrm{Br}_{m}}$. Группа $G(s)$ называется ассоциированной с элементом $s$.

В следующей хорошо известной лемме (см. доказательство, например, в [24]) описываются соотношения, заданные действием степеней некоторого полуповорота.

ЛЕмма 3.5. Пусть $b_{k}=g^{-1} a_{j}^{k+1} g \in \mathrm{Br}_{m}$. Тогда для каждого $k=0,1,2,-3$ множество соотношений

$$
\left\{x_{i}^{-1}\left(x_{i}\right) b_{k}=\mathbf{1} \mid i=1, \ldots, m\right\}
$$

в $\mathbb{F}_{m}$ эквивалентно единственному соотношению:

0) $\left(x_{j}\right) g=\left(x_{j+1}\right) g$, если $k=0$;

1) коммутант $\left[\left(x_{j}\right) g,\left(x_{j+1}\right) g\right]$ равен $\mathbf{1}$, если $k=1$ или -3 ;

2) $\left(x_{j}\right) g\left(x_{j+1}\right) g\left(x_{j}\right) g=\left(x_{j+1}\right) g\left(x_{j}\right) g\left(x_{j+1}\right) g$, если $k=2$.

В общем случае каждое соотношение $x_{i}=\left(x_{i}\right) b$ имеет вид $x_{i}=w_{i, b}^{-1} x_{\sigma_{b}(i)} w_{i, b}$, где $\sigma_{b} \in \mathscr{S}_{m}$ - некоторая перестановка, действующая на целых точках отрезка $[1, m]$, и $w_{i, b}=w_{i, b}\left(x_{1}, \ldots, x_{m}\right)$ - некоторое слово, состоящее из букв алфавита $\left\{x_{1}^{ \pm 1}, \ldots, x_{m}^{ \pm 1}\right\}$. Следовательно, каждая группа $G_{B}$ (и, в частности, $G(s)$ ) является $C$-группой.

3.5. $C$-группы. Согласно определению $C$-груnпа - это группа вместе с конечным копредставлением

$$
G_{W}=\left\langle x_{1}, \ldots, x_{m} \mid x_{i}=w_{i, j, k}^{-1} x_{j} w_{i, j, k}, w_{i, j, k} \in W\right\rangle,
$$

где $W=\left\{w_{i, j, k} \in \mathbb{F}_{m} \mid 1 \leqslant i, j \leqslant m, 1 \leqslant k \leqslant h(i, j)\right\}$ - это набор, состоящий из элементов свободной группы $\mathbb{F}_{m}$, свободно порожденной элементами $x_{1}, \ldots, x_{m}$ (возможно, что $w_{i_{1}, j_{1}, k_{1}}=w_{i_{2}, j_{2}, k_{2}}$ для $\left(i_{1}, j_{1}, k_{1}\right) \neq\left(i_{2}, j_{2}, k_{2}\right)$ ), a $h:\{1, \ldots, m\}^{2} \rightarrow \mathbb{Z}$ - некоторая функция. Такое копредставление называется $C$-копредставлением ( $C$ означает, что все соотношения имеют вид сопряжений). Пусть $\varphi_{W}: \mathbb{F}_{m} \rightarrow G_{W}-$ канонический эпиморфизм. Элементы $\varphi_{W}\left(x_{i}\right) \in G, 1 \leqslant i \leqslant m$, и элементы, сопряженные им, называются $C$-порождающими группы $G$. Пусть $f: G_{1} \rightarrow G_{2}$ - гомоморфизм $C$-групп. Он называется $C$-гомоморфизмом, если образы $C$-порождающих группы $G_{1}$ при гомоморфизме $f$ являются $C$-порождающими $C$-группы $G_{2}$. Будем рассматривать $C$-группы с точностью до $C$-изоморфизмов. Некоторые свойства $C$-групп были исследованы в [24]-[27].

Говорят, что $C$-копредставление

$$
G=\left\langle x_{1}, \ldots, x_{m} \mid r_{1}, \ldots, r_{n}\right\rangle
$$

является простым, если каждое соотношение $r_{j}$, входящее в копредставление (11), имеет вид

$$
x_{i_{3}}^{-1} x_{i_{1}} x_{i_{3}} x_{i_{2}}^{-1}=\mathbf{1}
$$

при некоторых $i_{1}, i_{2}, i_{3} \in\{1, \ldots, m\}$ (т. е. соотношение $r_{j}$ является соотношением вида $x_{i_{2}}=x_{i_{3}}^{-1} x_{i_{1}} x_{i_{3}}$ ). 
Лемма 3.6. Каждая $C$-группа $G$ обладает простым $C$-копредставлением.

ДоказАтельСтво. Пусть $r:=w^{-1} x_{i} w x_{j}^{-1}-$ одно из соотношений в $C$-копредставлении группы $G$ (т.е. $w^{-1} x_{i} w=x_{j}$ ), где $w=x_{i_{1}}^{\varepsilon_{1}} \ldots x_{i_{k}}^{\varepsilon_{k}}$ является словом в группе $\mathbb{F}_{m}, \varepsilon_{l}= \pm 1$. Тогда можно добавить $k-1$ новых порождающих элементов $x_{m+1}, \ldots, x_{m+k-1}$ и заменить соотношение $r$ на $k$ новых простых соотношений:

$$
\begin{aligned}
& x_{m+1}=x_{i_{1}}^{-\varepsilon_{1}} x_{i} x_{i_{1}}^{\varepsilon_{1}}, \\
& x_{m+2}=x_{i_{2}}^{-\varepsilon_{2}} x_{m+1} x_{i_{2}}^{\varepsilon_{2}}, \\
& \ldots \ldots \ldots \ldots \ldots \ldots \ldots \ldots \ldots \ldots \ldots \ldots \ldots \ldots \\
& x_{m+k-1}=x_{i_{k-1}}^{-\varepsilon_{k-1}} x_{m+k-2} x_{i_{k-1}}^{\varepsilon_{k-1}}, \\
& x_{j}=x_{i_{k}}^{-\varepsilon_{k}} x_{m+k-1} x_{i_{k}}^{\varepsilon_{k}} .
\end{aligned}
$$

Очевидно, что в результате получим новое $C$-копредставление, определяющее ту же $C$-группу $G$. Лемма 3.6 доказана.

Легко показать, что для любой $C$-группы $G$ группа $G / G^{\prime}$ является конечно порожденной свободной абелевой группой, где $G^{\prime}=[G, G]$ - коммутант группы $G$. $C$-группа $G$ называется неприводимой, если $G / G^{\prime} \simeq \mathbb{Z}$, и мы будем говорить, что $G$ составлена из $k$ неприводимљх компонент, если $G / G^{\prime} \simeq \mathbb{Z}^{k}$.

Примерами $C$-групп являются группы узлов и зацеплений (заданных копредставлениями Виртингера). Более того, следующая теорема является хорошо известной.

Теорема 3.3 [21]. Если зацепление $l$ представлено в виде замкнутой косъь $\bar{b}, b \in \mathrm{Br}_{m}$, mо группа $\pi_{1}\left(S^{3} \backslash l\right) C$-изоморфна $C$-группе $G_{\langle b\rangle}$, где $\langle b\rangle$ ииклическая подгруппа группъ $\mathrm{Br}_{m}$, порожденная элементом $b$.

Обобщенное копредставление Виртингера (см. [28]) фундаментальной группы $\pi_{1}\left(S^{n+2} \backslash V\right)$ дополнения к замкнутому ориентированному $n$-мерному многообразию $V$ без границы в $(n+2)$-мерной сфере $S^{n+2}$, состоящему из $k$ связных компонент, задает на $\pi_{1}\left(S^{n+2} \backslash V\right)$ структуру $C$-группы, составленной из $k$ неприводимых компонент, и, более того, каждая $C$-группа $G$ может быть реализована как фундаментальная группа $\pi_{1}\left(S^{n+2} \backslash V\right)$ для некоторого такого многообразия $V \subset S^{n+2}$ при любом $n \geqslant 2$.

Обозначим через $\mathscr{C}$ множество всех $C$-групп и через $\mathscr{C}_{\mathrm{Br}}=\cup\left\{G_{B}\right\} \subset \mathscr{C}$ множество всех $C$-групп, которые $C$-изоморфны группам $G_{B}$, где объединение взято по всем $m \in \mathbb{N}$ и всем конечно порожденным подгруппам $B$ групп $\mathrm{Br}_{m}$. Отметим, что множество $C$-групп $\mathscr{C}$ содержит очень много групп. Так, для каждой конечно определенной группы

$$
G=\left\langle x_{1}, \ldots, x_{m} \mid w_{i}\left(x_{1}, \ldots, x_{m}\right)=\mathbf{1}, i=1, \ldots, n\right\rangle
$$

найдется $C$-группа $\widetilde{G}$, доминирующая группу $G$, например,

$$
\widetilde{G}=\left\langle x_{1}, \ldots, x_{m} \mid\left[w_{i}\left(x_{1}, \ldots, x_{m}\right), x_{j}\right]=\mathbf{1}, i=1, \ldots, n, j=1, \ldots, m\right\rangle .
$$

Следующая теорема является центральной при доказательстве реализуемости каждой $C$-группы $G$ как фундаментальной группы дополнения в бидиске 
к пересечению некоторой неособой алгебраической кривой с этим бидиском (см. теорему 7.2).

Теорема 3.4 [24]. Для любой $C$-группы $G$ найдутся натуральное число $m$ и элемент $s \in S\left(\mathrm{Br}_{m}, A_{0}\right)$ такие, что группь $G u G(s)$ являются $C$-изоморфными. В частности, имеем равенство $\mathscr{C}_{\mathrm{Br}}=\mathscr{C}$.

ДокАЗАТЕЛЬСтво. Согласно лемме 3.6 группа $G$ обладает некоторым простым $C$-копредставлением $\left\langle x_{1}, \ldots, x_{m} \mid r_{1}, \ldots, r_{n}\right\rangle$. Очевидно, что копредставление

$$
\left\langle x_{1}, \ldots, x_{2 m} \mid r_{1}, \ldots, r_{n+m}\right\rangle
$$

также является $C$-копредставлением $C$-группы $G$, где $r_{1}, \ldots, r_{n}$ - это старые простые соотношения, а соотношение $r_{n+i}, i=1, \ldots, m,-$ это соотношение $x_{i+m}=x_{i}$ (которое может быть записано в виде $x_{i+m}=x_{i}^{-1} x_{i} x_{i}$ ). После этого каждое из соотношений $r_{j}, j \leqslant n$, имеющее вид $x_{i_{3}}=x_{i_{2}}^{-1} x_{i_{1}} x_{i_{2}}, i_{2}<i_{1}$, заменяем на соотношение $x_{i_{3}}=x_{i_{2}+m}^{-1} x_{i_{1}} x_{i_{2}+m}$ и получаем (ввиду наличия соотношений $\left.x_{i+m}=x_{i}, \quad i=1, \ldots, m\right)$ новое простое $C$-копредставление $\left\langle x_{1}, \ldots, x_{2 m} \mid r_{1}, \ldots, r_{n+m}\right\rangle$ группы $G$, в котором все соотношения $r_{j}$ имеют вид $x_{i_{3}}=x_{i_{2}}^{-1} x_{i_{1}} x_{i_{2}}, i_{2} \geqslant i_{1}$. Если в полученном $C$-копредставлении имеется соотношение вида $x_{i_{3}}=x_{i_{2}}^{-1} x_{i_{1}} x_{i_{2}}, i_{2}>i_{3}$, то повторим описанную выше процедуру удвоения числа порождающих элементов, добавив соотношения $x_{i+2 m}=x_{i}, \quad i=1, \ldots, 2 m$, и заменив все соотношения $r_{j}$, имеющие вид $x_{i_{3}}=x_{i_{2}}^{-1} x_{i_{1}} x_{i_{2}}, i_{2}>i_{3}$, на соотношения $x_{i_{3}+2 m}=x_{i_{2}}^{-1} x_{i_{1}} x_{i_{2}}$. В итоге получим $C$-копредставление $\left\langle x_{1}, \ldots, x_{4 m} \mid r_{1}, \ldots, r_{n+3 m}\right\rangle$ группы $G$, в котором каждое соотношение $r_{j}$ имеет вид $x_{i_{3}}=x_{i_{2}}^{-1} x_{i_{1}} x_{i_{2}}, i_{3}>i_{2} \geqslant i_{1}$. Применяя лемму 3.5 , получаем, что такое соотношение эквивалентно соотношениям $x_{i}=\left(x_{i}\right) b_{r_{j}}$, $i=1, \ldots, 4 m$, где $b_{r_{j}}=g_{1}^{-1} g_{2}^{-1} a_{i_{2}} g_{2} g_{1} \in A_{0} \subset \mathrm{Br}_{4 m}, g_{1}=a_{i_{3}-1} a_{i_{3}-2} \ldots a_{i_{2}+1}$, если $i_{3}>i_{2}+1$, и $g_{1}=\mathbf{1}$, если $i_{3}=i_{2}+1$, а $g_{2}=a_{i_{2}-1}^{-1}\left(a_{i_{2}-2} a_{i_{2}}-3 \ldots a_{i_{1}}\right)$, если $i_{2} \geqslant i_{1}+2, g_{2}=a_{i_{2}-1}^{-1}$, если $i_{2}=i_{1}+1$, и $g_{2}=\mathbf{1}$, если $i_{2}=i_{1}$. Следовательно, группа $G C$-изоморфна группе $G(s)$, где $s=b_{r_{1}} \cdot \ldots \cdot b_{r_{n+3 m}}$. Теорема 3.4 доказана.

ПРЕДЛОЖЕНИЕ 3.1. Для $s_{1}, s_{2} \in S_{B r_{m}}$ имеем:

(i) существует канонический $C$-эпиморфизм $\psi_{s_{1}}: G\left(s_{2}\right) \rightarrow G\left(s_{1} \cdot s_{2}\right)$;

(ii) если $s_{2}=\lambda(g)\left(s_{1}\right)$, то существует $C$-изоморфизм групп $\gamma_{g}: G\left(s_{1}\right) \rightarrow$ $G\left(s_{2}\right)$, в частности, если $s_{1}=s_{2}$, mо $G\left(s_{1}\right)=G\left(s_{2}\right)$ u $\gamma_{\mathbf{1}}=\mathrm{Id}$.

ДоказАтельство. Пусть $s_{1}=b_{1} \cdot \ldots \cdot b_{n}$. Очевидно, что имеет место изоморфизм $G\left(s_{1} \cdot s_{2}\right) \simeq G\left(s_{2}\right) / N$, где $N$ - нормальная подгруппа в $G\left(s_{2}\right)$, нормально порожденная образами элементов $\left(x_{i}\right) b_{j} x_{i}^{-1}, i=1, \ldots, m$ и $j=1, \ldots, n$.

Если $s_{2}=\left(g^{-1} b_{1} g\right) \cdot \ldots \cdot\left(g^{-1} b_{n} g\right)$, то

$$
G\left(s_{1}\right)=\left\langle x_{1}, \ldots, x_{m} \mid x_{i}=\left(x_{i}\right) b_{j}\right\rangle \quad \text { и } G\left(s_{2}\right)=\left\langle x_{1}, \ldots, x_{m} \mid x_{i}=\left(x_{i}\right) g^{-1} b_{j} g\right\rangle .
$$

В новом базисе $y_{1}=\left(x_{1}\right) g, \ldots, y_{m}=\left(x_{m}\right) g$ в $\mathbb{F}_{m}$ группа $G\left(s_{2}\right)$ будет иметь копредставление $G\left(s_{2}\right)=\left\langle x_{1}, \ldots, x_{m} \mid\left(x_{i}\right) g=\left(x_{i}\right) b_{j} g\right\rangle$ и, следовательно, автоморфизм $g: \mathbb{F}_{m} \rightarrow \mathbb{F}_{m}$ индуцирует $C$-изоморфизм $g_{*}: G\left(s_{1}\right) \rightarrow G\left(s_{2}\right)$. 
3.6. Гурвицевские $C$-группы. Группы, принадлежащие подмножеству множества $C$-групп, описанию которых посвящен этот пункт, имеют естественную геометрическую реализацию, а именно, они реализуются как фундаментальные группы дополнений к “аффинным” кривым Гурвица (см. §7).

$C$-копредставление (10) называется гурвицевским $C$-копредставлением степени $m$, если существуют такие $C$-порождающие $x_{1}, \ldots, x_{m}$, что они порождают группу $G$ и произведение $x_{1} \ldots x_{m}$ принадлежит центру группы $G$. Такую $C$-группу будем называть гурвицевской $C$-группой степени $m$. Отметим, что степень гурвицевской $C$-группы $G$ определена не канонически и она зависит от гурвицевского $C$-копредставления группы $G$. Обозначим через $\mathscr{H}$ множество всех гурвицевских $C$-групп (рассматриваемых с точностью до $C$-изоморфизма).

ЛЕмма 3.7. Если конечно порожденная подгруппа $B \subset \mathrm{Br}_{m}$ содержит элемент $\Delta_{m}^{2 k}$ при некотором $k \in \mathbb{N}$, то группа $G_{B}$ является гурвицевской $C$-группой степени $\mathrm{mk}$.

Действительно, группа $G_{B}$ может быть задана $C$-копредставлением

$$
G_{B}=\left\langle x_{1}, \ldots, x_{m} \mid x_{i}=\left(x_{i}\right) b_{j}, 1 \leqslant i \leqslant m, 1 \leqslant j \leqslant n\right\rangle,
$$

где $b_{1}, \ldots, b_{n}$ - порождающие элементы группы $B$. Элемент $\left(x_{1} \ldots x_{m}\right)^{k}$ принадлежит центру группы $G_{B}$, так как $\Delta_{m}^{2 k} \in B$ и $\left(x_{i}\right) \Delta_{m}^{2}=\left(x_{1} \ldots x_{m}\right) x_{i}\left(x_{1} \ldots x_{m}\right)^{-1}$ для всех $i$. Добавив новые порождающие элементы $x_{m+1}, \ldots, x_{m k}$ и соотношения $x_{i}=x_{i+m}, i=1, \ldots, m(k-1)$, в $C$-копредставление $(12)$, получим новое $C$-копредставление, определяющее ту же $C$-группу $G_{B}$. Это $C$-копредставление является гурвицевским степени $m k$, так как в группе $G_{B}$ имеет место равенство: $x_{1} \ldots x_{m k}=\left(x_{1} \ldots x_{m}\right)^{k}$.

Теорема 3.5 [24]. Для любой гурвичевской $C$-группы $G$ степени $m$ найдутся число $n \in \mathbb{N}$ и элемент $s \in S\left(\mathrm{Br}_{2^{n}} m, A_{0} \cup O_{\Delta_{m}^{2}}\right)$ такие, что

(i) $\alpha(s)=\Delta_{2^{n} m}^{2}$;

(ii) группа $G(s) C$-изоморфна группе $G$.

Доказательство этой теоремы существенно использует формулу разложения на множители полного поворота с удвоенным числом нитей и в основном аналогично доказательству теоремы 3.4 (см. подробности в [24]). Эта теорема является центральной при доказательстве реализуемости каждой гурвицевской $C$-группы $G$ как фундаментальной группы дополнения к некоторой “аффинной” кривой Гурвица (см. теорему 7.3).

3.7. Модули Александера и многочлены Александера $C$-групп. Согласно предложению $3.1 C$-группы $G\left(s_{1}\right)$ и $G\left(s_{2}\right)$, ассоциированные с элементами $s_{1}, s_{2} \in S_{\mathrm{Br}_{m}}$, принадлежащими одной и той же орбите действия сопряжением группы $\mathrm{Br}_{m}$ на полугруппе $S_{\mathrm{Br}_{m}}$, являются $C$-изоморфными группами. Поэтому чтобы различить разные орбиты этого действия, мы можем использовать ассоциированные с элементами $s \in S_{\mathrm{Br}_{m}}$ группы $G(s)$ и связанные с ними объекты в качестве инвариантов орбит. Одними из важных объектов, связанных с $C$-группой, являются ее модуль и многочлен Александера. Напомним их определения в случае $C$-групп. 
Свободная группа $\mathbb{F}_{n}$ с фиксированными свободными порождающими является $C$-группой, и для любой $C$-группы $G$ определен канонический $C$-эпиморфизм $\nu: G \rightarrow \mathbb{F}_{1}$, отображающий $C$-порождающие элементы группы $G$ в $C$-порождающий элемент группы $\mathbb{F}_{1}$. Обозначим через $N$ его ядро. Отметим, что если $G$ является неприводимой $C$-группой, то $N$ совпадает с $G^{\prime}$, где $G^{\prime}=[G, G]-$ коммутант группы $G$.

В случае гурвицевских $C$-групп ядро $N$ является конечно определенной группой (см. [27]). Однако это не так для произвольных $C$-групп. Например, коммутант группы узла является конечно порожденной группой тогда и только тогда, когда узел является расслоенным узлом (см. [29]).

$C$-эпиморфизм $\nu$ индуцирует следующую точную последовательность групп:

$$
1 \rightarrow N / N^{\prime} \rightarrow G / N^{\prime} \stackrel{\nu_{*}}{\rightarrow} \mathbb{F}_{1} \rightarrow 1
$$

$C$-порождающий элемент группы $\mathbb{F}_{1}$ действует на группе $N / N^{\prime}$ сопряжениями: $g \mapsto \tilde{x}^{-1} g \tilde{x}$, где $g \in N$ и $x$ - это один из $C$-порождающих элементов группы $G$, а $\tilde{x}$ - его образ в $G / N^{\prime}$. Обозначим это действие через $t$. Группа $A_{0}(G)=N / N^{\prime}$ является абелевой группой, и действие $t$ определяет на $A_{0}(G)$ структуру $\Lambda$-модуля, где $\Lambda=\mathbb{Z}\left[t, t^{-1}\right]$ - это кольцо многочленов Лорана с целыми коэффициентами. $\Lambda$-модуль $A_{0}(G)$ называется модулем Александера $C$-группы $G$. Действие $t$ индуцирует действие $h_{\mathbb{C}}$ на $A_{\mathbb{C}}=A_{0}(G) \otimes \mathbb{C}$, и легко видеть, что его характеристический многочлен $\operatorname{det}\left(h_{\mathbb{C}}-t \mathrm{Id}\right) \in \mathbb{Q}[t]$. Многочлен $\Delta(t)=a \operatorname{det}\left(h_{\mathbb{C}}-t \mathrm{Id}\right)$, где $a \in \mathbb{N}$ - наименьшее натуральное число такое, что $a \operatorname{det}\left(h_{\mathbb{C}}-t \mathrm{Id}\right) \in \mathbb{Z}[t]$, называется многочленом Александера $C$-группы $G$ (если векторное пространство $N / N^{\prime} \otimes \mathbb{C}$ над $\mathbb{C}$ является бесконечномерным, то по определению многочлен Александера $\Delta(t) \equiv 0)$.

Так как $C$-группы $G$ являются конечно порожденными, то их модули Александера $A_{0}(G)$ являются нётеровыми $\Lambda$-модулями.

3.8. Вычисление модулей Александера $C$-групп. Один из основных методов вычисления модулей Александера $C$-групп основан на свободном дифференцировании Фокса. Напомним кратко этот метод. Пусть

$$
G=\left\langle x_{1}, \ldots, x_{m} \mid r_{1}=1, \ldots, r_{n}=1\right\rangle
$$

- $C$-копредставление $C$-группы $G$ и $\mathbb{F}_{m}$ - свободная группа, свободно порожденная $C$-порождающими элементами $x_{1}, \ldots, x_{m}$. Обозначим через $\frac{\partial}{\partial x_{i}}$ дифференцирование Фокса [30], т. е. эндоморфизм группового кольца $\mathbb{Z}\left[\mathbb{F}_{m}\right]$ над $\mathbb{Z}$ свободной группы $\mathbb{F}_{m}$ в себя такой, что $\frac{\partial}{\partial x_{i}}: \mathbb{Z}\left[\mathbb{F}_{m}\right] \rightarrow \mathbb{Z}\left[\mathbb{F}_{m}\right]$ является $\mathbb{Z}$-линейным отображением таким, что

$$
\begin{aligned}
& \frac{\partial x_{j}}{\partial x_{i}}=\delta_{i, j} \\
& \frac{\partial u v}{\partial x_{i}}=\frac{\partial u}{\partial x_{i}}+u \frac{\partial v}{\partial x_{i}}
\end{aligned}
$$

для всех $u, v \in \mathbb{Z}\left[\mathbb{F}_{m}\right]$. 
Матрица

$$
\mathscr{A}(G)=\nu_{*}\left(\frac{\partial r_{i}}{\partial x_{j}}\right) \in \operatorname{Mat}_{n \times m}\left(\mathbb{Z}\left[t, t^{-1}\right]\right)
$$

называется матрицей Александера $C$-группы $G$, заданной копредставлением (13), где $r_{i}, i=1, \ldots, n,-$ определяющие соотношения группы $G$ и гомоморфизм $\nu_{*}: \mathbb{Z}\left[\mathbb{F}_{m}\right] \rightarrow \mathbb{Z}\left[\mathbb{F}_{1}\right] \simeq \mathbb{Z}\left[t, t^{-1}\right]$ индуцирован каноническим $C$-эпиморфизмом $\nu: \mathbb{F}_{m} \rightarrow \mathbb{F}_{1}$.

Каждое соотношение $r_{i}$ имеет вид

$$
r=w x_{j} w^{-1} x_{l}^{-1}
$$

где $w$ - это некоторое слово, состоящее из букв $x_{1}^{ \pm 1}, \ldots, x_{m}^{ \pm 1}$, и $x_{j}, x_{l}$ - некоторые две буквы. Применяя индукцию по длине $\ln (w)$ слова $w$, легко доказать справедливость следующей леммы.

Лемма 3.8. Сумма столбцов матрицы Александера $\mathscr{A}(G) C$-группы $G$, заданной копредставлением (13), равна нулю.

Доказательство следующего предложения, по существу, повторяет доказательство аналогичного утверждения для модулей Александера зацеплений (см. [31]).

ПреДЛОЖЕНИЕ 3.2 [32]. Модуль Александера $A_{0}(G) C$-группы $G$, заданной копредставлением (13), изоморфен фактормодулю $\Lambda^{m-1} / M(G)$, где подмодуль $M(G)$ модуля $\Lambda^{m-1}$ порожден строками матрицы $\overline{\mathscr{A}}$, сбормированной из первых $m-1$ столбцов матрищы Александера $\mathscr{A}(G)$.

Если $f: G_{1} \rightarrow G_{2}-C$-эпиморфизм $C$-групп $G_{1}$ и $G_{2}$, то в коммутативной диаграмме

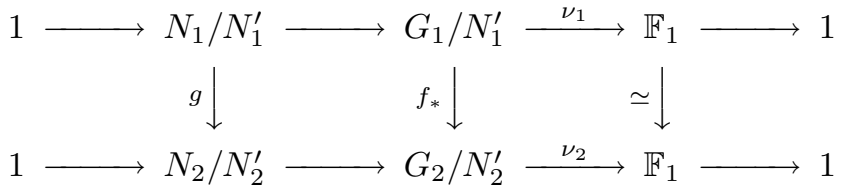

гомоморфизм $g$ является эпиморфизмом. Отсюда следует, что в этом случае модуль Александера $A_{0}\left(G_{2}\right)$ является фактормодулем модуля Александера $A_{0}\left(G_{1}\right)$, а многочлен Александера $\Delta_{2}(t)$ группы $G_{2}$ (если он не равен тождественно нулю) является делителем многочлена Александера $\Delta_{1}(t)$ группы $G_{1}$.

В частности, каждая гурвицевская $C$-группа $G$ степени $m$ является эпиморфным образом гурвицевской $C$-группы

$$
\widetilde{G}_{m}=\left\langle x_{1}, \ldots, x_{m} \mid\left[\left(x_{1} \ldots x_{m}\right), x_{i}\right]=\mathbf{1}, i=1, \ldots, m\right\rangle .
$$

Применив метод Рейдемейстера-Шрайера (см., например, [33]) для нахождения порождающих элементов ядра $N$ эпиморфизма $\nu: \widetilde{G}_{m} \rightarrow \mathbb{F}_{1}$ и соотношений между ними, можно показать, что модуль Александера $A_{0}\left(\widetilde{G}_{m}\right)$ группы $\widetilde{G}_{m}$ изоморфен $\Lambda$-модулю $\left(\Lambda /\left(t^{m}-1\right) \Lambda\right)^{m-2} \oplus(\Lambda /(t-1) \Lambda)$. В качестве следствия получаем следующий результат. 
ПредЛОЖенИЕ 3.3 [15]. Многочлен Александера $\Delta(t)$ гурвицевской $C$-груп$n$ и $G$ степени $m$ является делителем многочлена $(t-1)\left(t^{m}-1\right)^{m-2}$. В частности, все корни многочлена $\Delta(t)$ являются корнями $m$-й степени из единицы.

$\Lambda$-модуль $M$ называется $t$-унипотентным, если для некоторого $n \in \mathbb{N}$ умножение на $t^{n}$ является тождественным автоморфизмом модуля $M$. Наименьшее $k \in \mathbb{N}$ такое, что

$$
t^{k}-1 \in \operatorname{Ann}(M)=\{f(t) \in \Lambda \mid f(t) v=0 \forall v \in M\},
$$

называется индексом унипотентности $t$-унипотентного модуля $M$.

Из вышесказанного следует, что модули Александера гурвицевских $C$-групп обладают следующим замечательным свойством.

ПреДЛОЖЕНИЕ 3.4 [32]. Модуль Александера $A_{0}(G)$ гурвицевской $C$-группь $G$ степени $m$ является нётеровым $t$-унипотентным $\Lambda$-модулем индекса унипотентности $d$, где $d$ - некоторый делитель числа $m$.

Пусть гурвицевская $C$-группа составлена из $k$ неприводимых компонент. Эпиморфизм $\mathrm{ab}: G \rightarrow G / G^{\prime} \simeq \mathbb{Z}^{k}$ является $C$-эпиморфизмом (если в качестве $C$-порождающих элементов группы $G / G^{\prime}$ взять образы $C$-порождающих элементов группы $G)$. Очевидная проверка показывает, что модуль Александера $A_{0}\left(\mathbb{Z}^{k}\right)$ изоморфен $(\Lambda /(t-1) \Lambda)^{k-1}$. Отсюда следует, что многочлен $(t-1)^{k-1}$ является делителем многочлена Александера $\Delta(t)$ гурвицевской $C$-группы $G$, составленной из $k$ неприводимых компонент. Можно показать, что верно и обратное утверждение, т. е. имеет место следующее предложение.

ПреДЛОЖЕНИЕ 3.5 [15]. Многочлен Александера $\Delta(t)$ гурвищевской $C$-груп$n$ и $G$, составленной из $k$ неприводимых компонент, равен $\Delta(t)=(t-1)^{k-1} P(t)$, где $P(t)$ - некоторый многочлен такой, что $P(1) \neq 0$.

Наиболее хорошо изучены модули Александера неприводимых $C$-групп.

3.9. Модули Александера неприводимых $C$-групп. Пусть $M$ - нётеров $\Lambda$-модуль. Скажем, что $M$ является $(t-1)$-обратимым, если умножение на $t-1$ является автоморфизмом модуля $M$.

Для неприводимой $C$-группы $G$ ее коммутант $G^{\prime}$ совпадает с ядром $C$-эпиморфизма $\nu: G \rightarrow \mathbb{F}_{1}$. Применив метод Рейдемейстера-Шрайера, можно доказать следующую лемму.

Лемма 3.9 [34]. Модуль Александера $A_{0}(G)=G^{\prime} / G^{\prime \prime}$ неприводимой $C$-группы $G$ является нётеровым $(t-1)$-обратимым $\Lambda$-модулем.

Оказывается, верно и обратное утверждение.

Теорема 3.6 [32]. $\Lambda$-модуль $M$ является модулем Александера некоторой неприводимой $C$-группы тогда и только тогда, когда $M$ является нётеровым $(t-1)$-обратимым $\Lambda$-модулем.

Аналогичное утверждение также верно и для неприводимых гурвицевских C-групп. 
Теорема 3.7 [32]. $\Lambda$-модуль $M$ является модулем Александера некоторой неприводимой гурвичевской $C$-группы тогда и только тогда, когда $M$ является нётеровым $(t-1)$-обратимым

Для доказательства в теоремах 3.6 и 3.7 существования $C$-группы $G$, модуль Александера $A_{0}(G)$ которой изоморфен заданному $\Lambda$-модулю $M$, применяется следующее предложение.

ПрЕДЛОЖЕНИЕ 3.6. Каждый нётеров $(t-1)$-обратимый $\Lambda$-модуль $M$ изоморфен фактормодулю $\Lambda^{n} / M_{1}$ свободного $\Lambda$-модуля $\Lambda^{n}$, где подмодуль $M_{1}$ порождается элементами $w_{1}, \ldots, w_{n}, \ldots, w_{n+k}$ модуля $\Lambda^{n}$ такими, что:

(i) для $i=1, \ldots, n$ вектор $w_{i}$ равен $\left(0, \ldots, 0, f_{i}(t), 0, \ldots, 0\right)$, где многочлен $f_{i}(t)$ cmoum на $i$-м месте $u f_{i}(1)=1$;

(ii) $w_{n+j}=(t-1) \bar{w}_{n+j}=\left((t-1) g_{j, 1}(t), \ldots,(t-1) g_{j, n}(t)\right)$ для $j=1, \ldots, k$, где $g_{j, l}(t)$ - некоторые многочлены;

(iii) если для некоторого $m \in \mathbb{N}$ многочлен $t^{m}-1 \in \operatorname{Ann}(M)$, то для $i=$ $1, \ldots, n$ вектор $w_{n+i}$ равен $\left(0, \ldots, 0, t^{m}-1,0, \ldots, 0\right)$, где многочлен $t^{m}-1$ cmoum на $i$-м месте.

Затем показывается, что для каждого вектора $\omega_{i}$ из предложения 3.6 найдется такое $C$-соотношение $r_{i}$, что $\omega_{i}=\left(\nu_{*}\left(\frac{\partial r_{i}}{\partial x_{1}}\right), \ldots, \nu_{*}\left(\frac{\partial r_{i}}{\partial x_{n}}\right)\right)$, и после этого применяется предложение 3.2 .

3.10. Свойства нётеровых $(t-1)$-обратимых $\Lambda$-модулей. Прежде всего отметим, что условие $t-1 \in \operatorname{Aut}(M)$ в определении $(t-1)$-обратимости нётерова $\Lambda$-модуля $M$ можно ослабить. А именно, легко доказать, что нётеров $\Lambda$-модуль $M$ является $(t-1)$-обратимым тогда и только тогда, когда умножение на $t-1$ является сюръективным эндоморфизмом модуля $M$. Кроме того, простая проверка показывает, что каждый подмодуль и каждый фактормодуль нётерова $(t-1)$-обратимого $\Lambda$-модуля $M$ является нётеровым $(t-1)$-обратимым модулем.

В качестве замечания отметим, что абелева группа $G$ допускает структуру $(t-1)$-обратимого $\Lambda$-модуля тогда и только тогда, когда она имеет автоморфизм $t$ такой, что $t-1$ также является автоморфизмом. Если группа $G$ конечно порождена и выбран такой автоморфизм $t \in \operatorname{Aut} G$, то $G$ является нётеровым $\Lambda$-модулем. Заметим, что в общем случае абелева группа допускает много различных структур $(t-1)$-обратимых $\Lambda$-модулей. Например, группа $\mathbb{Z} / 9 \mathbb{Z}$ допускает три таких структуры: либо $t v=2 v$, либо $t v=5 v$, либо $t v=8 v$, где $v-$ порождающий элемент группы $\mathbb{Z} / 9 \mathbb{Z}$.

Следующее предложение дает критерий $(t-1)$-обратимости нётерова $\Lambda$-модуля.

ПРЕДЛОЖЕНИЕ 3.7 [32]. Нётеров $\Lambda$-модуль $M$ является $(t-1)$-обратимыъм тогда и только тогда, когда найдется многочлен $f(t) \in \operatorname{Ann}(M)$ такой, что $f(1)=1$.

Отсюда вытекает следующее утверждение.

СлеДСТвИЕ 3.1. Пусть $\Delta(t)$ - многочлен Александера неприводимой $C$-группъц $G$. Тогда $\Delta(1)= \pm 1$. 
Применяя предложение 3.6 и теорему 3.6, легко показать, что для любого многочлена $P(t) \in \mathbb{Z}[t]$ такого, что $P(1)= \pm 1$, существует неприводимая $C$-группа $G$, многочлен Александера которой $\Delta(t)$ равен $\pm P(t)$.

Из следствия 3.1 вытекает, что корни уравнения $t^{\left(p^{k}\right)}-1=0$, где $p-$ простое число, не могут быть корнями многочленов Александера неприводимых $C$-групп. Отсюда и из теоремы 3.3 получаем

СлеДСтвИЕ 3.2. Многочлен Александера $\Delta(t)$ неприводимой гурвицевской $C$-группи $G$ степени $p^{k}$, где $p$ - простое число, тождественно равен единице.

Заметим, что аналогичное утверждение о корнях многочлена Александера гурвицевских $C$-групп, составленных более чем из одной неприводимой компоненты, уже не имеет места. Так, например, многочлен Александера $\Delta(t)$ гурвицевской $C$-группы, заданной $C$-копредставлением

$$
\begin{gathered}
G=\left\langle x_{1}, \ldots, x_{4}\right| x_{2}^{2} x_{1} x_{2}^{-2}=x_{4}, x_{3}=x_{2}, x_{4}^{2} x_{2} x_{4}^{-2}=x_{2}, \\
\left.\left[x_{i}, x_{1} \ldots x_{4}\right]=1 \text { для } i=1, \ldots, 4\right\rangle,
\end{gathered}
$$

равен $t^{2}-1$ (см. [15]).

Из предложения 3.7 вытекает, что каждый нётеров $(t-1)$-обратимый модуль $M$ является $\Lambda$-модулем кручения и, следовательно,

$$
\operatorname{dim}_{\mathbb{Q}} M \otimes \mathbb{Q}<\infty
$$

В общем случае нётеровы $(t-1)$-обратимые $\Lambda$-модули не являются конечно порожденными $\mathbb{Z}$-модулями. Критерий конечной порожденности над $\mathbb{Z}$ дает следующее предложение.

ПРЕДЛОЖЕНИЕ 3.8 [32]. Нётеров $(t-1)$-обратимый $\Lambda$-модуль $M$ конечно порожден над $\mathbb{Z}$ тогда и только тогда, когда найдется такой многочлен

$$
q(t)=\sum_{i=0}^{n} a_{i} t^{i} \in \operatorname{Ann}(M),
$$

что $a_{n}=a_{0}=1$.

В частности, имеет место следующая теорема.

Теорема 3.8 [32]. Конечно порожденная абелева группа

$$
G=\mathbb{Z}^{k} \oplus G_{1} \oplus\left(\bigoplus_{i=1}^{n}\left(\mathbb{Z} / 2^{r_{i}} \mathbb{Z}\right)^{m_{i}}\right),
$$

где $r_{i} \neq r_{j}$ при $i \neq j$ и $G_{1}$ - это группа нечетного порядка, имеет структуру $(t-1)$-обратимого $\Lambda$-модуля тогда и только тогда, когда $k \geqslant 2 u m_{i} \geqslant 2$ для всех $i=1, \ldots, n$.

Из определения нётерова $(t-1)$-обратимого $t$-унипотентного $\Lambda$-модуля $M$ следует, что для некоторого $k \in \mathbb{Z}$ многочлен $t^{k}-1 \in \operatorname{Ann}(M)$. Поэтому предложение 3.8 влечет следующую теорему. 
ТЕОРема 3.9. Каждый нётеров $(t-1)$-обратимый $t$-унипотентный $\Lambda$-модуль $M$ конечно порожден над $\mathbb{Z}$.

Из теоремы 3.9 и структурной теоремы для конечно порожденных $\mathbb{Z}$-модулей следует, что для нётерова $(t-1)$-обратимого $t$-унипотентного $\Lambda$-модуля $M$ имеет место изоморфизм $\mathbb{Z}$-модулей:

$$
M \simeq M_{\text {fin }} \oplus \mathbb{Z}^{k}
$$

где $M_{\text {fin }}$ - подмодуль модуля $M$, состоящий из элементов конечного порядка. Ранк $k$ свободной части модуля $M$ в разложении (15) называется числом Бет$m u$ нётерова $(t-1)$-обратимого $t$-унипотентного $\Lambda$-модуля $M$.

Многочлен Александера нётерова $(t-1)$-обратимого $t$-унипотентного $\Lambda$-модуля $M$ не имеет вещественных корней. Поэтому имеет место следующая теорема.

Теорема 3.10. Число Бетти нётерова $(t-1)$-обратимого $t$-унипотентного $\Lambda$-модуля $M$ является четным числом.

\section{§4. Разложение на множители брэйд-монодромии кривой Гурвица}

\section{1. Локальная брэйд-монодромия над точкой (случай одного рост-} ка). Здесь и далее мы обозначаем через $(u, v)$ стандартные координаты в $\mathbb{C}^{2}$. Пусть $o=(0,0)$ - начало координат в $\mathbb{C}^{2}$. Доказательства (хорошо известных) утверждений о монодромии аналитических особенностей, используемых здесь и ниже, могут быть найдены, например, в [35].

Если росток $(C, o) \subset\left(\mathbb{C}^{2}, o\right)$ приведенной комплексно-аналитической кривой не содержит ростка прямой $u=0$, то он может быть задан уравнением $P(u, v)=0$, где

$$
P(u, v)=v^{k}+\sum_{i=1}^{k} q_{i}(u) v^{k-i},
$$

а $q_{i}(u)$ - сходящиеся степенные ряды (т. е. $\left.q_{i}(u) \in \mathbb{C}\{u\}\right), q_{i}(0)=0$, и многочлен $v^{k}+\sum q_{i}(u) v^{k-i} \in \mathbb{C}\{u\}[v]$ не имеет кратных множителей. Поэтому можно выбрать маленький бидиск $D=D_{1} \times D_{2} \subset \mathbb{C}^{2}$, где $D_{1}=D_{1}\left(\varepsilon_{1}\right)=\{u \in \mathbb{C} \mid$ $\left.|u|<\varepsilon_{1}\right\}$ и $D_{2}=D_{2}\left(\varepsilon_{2}\right)=\left\{v \in \mathbb{C}|| v \mid<\varepsilon_{2}\right\}$, такой, что: $C$ является аналитическим множеством в замыкании $\bar{D}$ бидиска $D$, проекция на $u$-множитель $\mathrm{pr}=\operatorname{pr}_{1}: C \cap D \rightarrow D_{1}$ является собственным конечным отображением степени $k$ и $(u, v)=0$ является единственной критической точкой проекции $\operatorname{pr}_{\mid C \cap \bar{D}}$. Обратно, если $D$ - бидиск и $C-W$-подготовленный росток в $D$, т. е. если $C-$ приведенная комплексно-аналитическая кривая с перечисленными выше свойствами относительно pr, то она может быть задана в $\bar{D}$ уравнением такого типа. $W$-подготовленный росток $(C, o)$ является алгебраическим (в координатах $(u, v))$ тогда и только тогда, когда $q_{i} \in \mathbb{C}[u]$ для каждого $i=1, \ldots, k$. Чтобы определить брэйд-монодромию (монодромию в косах), выберем точку $p \in \partial D_{1}$ и положим $D_{2, p}=\operatorname{pr}^{-1}(p), K=K(p)=\left\{v_{1}, \ldots, v_{k}\right\}=D_{2, p} \cap C$. Петля $\partial D_{1}$ с ориентацией против часовой стрелки и с началом в точке $p$ поднимается на $\partial D \cap C$ и определяет траекторию $k$ различных точек $\operatorname{pr}_{2}\left(\left\{v_{1}(t), \ldots, v_{k}(t)\right\}\right)$ 
в $D_{2}$, начинающуюся и оканчивающуюся в $K$. Эта траектория определяет косу $b_{(C, o)} \in \operatorname{Br}_{k}=\operatorname{Br}_{k}\left[D_{2}, K\right]$, которая называется брэйд-монодромией ростка $(C, o)$ относительно проекиии $\mathrm{pr}$.

Лемма 4.1. Пусть $(C$, о - росток алгебраической кривой, заданной уравнением $v^{k}-u^{n}=0$. Тогда в группе $\mathrm{Br}_{k}=\operatorname{Br}_{k}\left[D_{2},\left\{e^{-2 \pi m i / k}\right\}_{m=0, \ldots, k-1}\right]$ брэйд-монодромия ростка $\left(C\right.$, o) относительно проекиии pr равна $\left(a_{1} \ldots a_{k-1}\right)^{n}$, где $a_{1}, \ldots, a_{k-1}$ - положительные полуповороты, ассоциированные с отрезками $l_{j}=\left[p_{j}, p_{j+1}\right]$ прямых, соединяющих точки $p_{j}=e^{-2 \pi(j-1) i / k} u p_{j+1}=e^{-2 \pi j i / k}$, $j=1, \ldots, k$.

Проиллюстрируем доказательство этой леммы на частном примере, когда $k=2$.

ПримеР 4.1. Пусть $C$ - алгебраическая кривая в аффинной плоскости $\mathbb{C}^{2}$, заданная уравнением $v^{2}=u^{n}$. Положим $\varepsilon_{1}=1, \varepsilon_{2}=2$ и $p=\{u=1\}$. Тогда $K=\{1,-1\}$ и $b_{(C, o)}=a_{1}^{n}$, где $a_{1}-$ положительный полуповорот, заданный путем $[1,-1]$. Действительно, имеем $\partial D_{1}=\left\{e^{2 \pi i t}, t \in[0,1]\right\}$. Поднимая $\partial D_{1}$ на $C$, получим два пути:

$$
\begin{aligned}
& \delta_{1}(t)=\left(e^{2 \pi i t}, e^{2 \pi i n t / 2}\right), \\
& \delta_{2}(t)=\left(e^{2 \pi i t},-e^{2 \pi i n t / 2}\right) .
\end{aligned}
$$

Проектируя $\delta_{1}(t), \delta_{2}(t)$ на $D_{2}$, получаем два пути:

$$
\begin{aligned}
& b_{(C, o), 1}(t)=e^{\pi i t \cdot n}, \\
& b_{(C, o), 2}(t)=-e^{\pi i t \cdot n},
\end{aligned}
$$

$0 \leqslant t \leqslant 1$, начинающиеся и заканчивающиеся в точках $\{1,-1\} \subset D_{2}$. Легко видеть, что эти пути (как нити косы в $\left.B_{2}\left[D_{2},\{1,-1\}\right]\right)$ являются $n$-й степенью косы $a_{1} \in B_{2}\left[D_{2},\{1,-1\}\right]$, состоящей из нитей

$$
\begin{aligned}
& a_{1,1}(t)=e^{\pi i t}, \\
& a_{1,2}(t)=-e^{\pi i t},
\end{aligned}
$$

$0 \leqslant t \leqslant 1$. Таким образом, $b_{(C, o)}=a_{1}^{n}$.

Аналогично, легко видеть, что брэйд-монодромия ростка $(H, o)$ отрицательного ноуда, заданного уравнением $v^{2}-\bar{u}^{2}=0$, относительно проекции pr равна $b_{(H, o)}=a_{1}^{-2}$.

В общем случае алгебраической кривой линк ростка $(C, o)$ является положительным итерированным торическим зацеплением. Этот линк определяется парами Пюизо неприводимых компонент ростка $(C, o)$ и взаимными индексами пересечений компонент. Поэтому как только мы выбираем в $\mathrm{Br}_{k}$ в качестве стандартных образующих положительные полуповороты $a_{1}, \ldots, a_{k-1}$, коса $b_{(C, o)}$ становится элементом, принадлежащим $\mathrm{Br}_{k}^{+}$(становится положительной косой). Назовем ее образ $\nu\left(b_{(C, 0)}\right) \in S_{\mathrm{Br}_{k}}$ стандартной формой брэйд-монодромии ростка $(C, o)$ относителъно pr. Стандартные образующие и, таким образом, стандартная форма $b_{(C, o)}$ определены однозначно с точностъю до сопряжения. 
Топологический тип тройки $(D, C, \mathrm{pr})$ определяется ее стандартной формой брэйд-монодромии, верно и обратное. Кроме того, для каждой тройки $(D, C, \mathrm{pr})$ найдется константа $M=M_{(C, o)} \in \mathbb{N}$ такая, что топологический тип тройки $(D, C, \mathrm{pr})$ совпадает с топологическим типом особенности, заданной уравнением $\bar{P}(u, v)=v^{k}+\sum \bar{q}_{i}(u) v^{k-i}=0$, где $\bar{q}_{i}(u)=q_{i}(u)+u^{M} r_{i}(u)$ и $r_{i}(u)$ - произвольные аналитические функции. В частности, существует алгебраическая тройка, имеющая тот же топологический тип.

Топологический тип тройки $(D, C, \mathrm{pr})$ и, таким образом, стандартная форма ее брэйд-монодромии определяются ее разрешением особенности относительно pr. Под последним мы понимаем последовательность таких раздутий $\sigma_{1}: U_{1} \rightarrow D, \ldots, \sigma_{n}: U_{n} \rightarrow U_{n-1}$ с центрами в точках, что $\sigma^{-1}(C \cup R)$ является дивизором с нормальными пересечениями, где $\sigma=\sigma_{n} \circ \cdots \circ \sigma_{1}$ и $R=\{u=0\}$. Положим

$$
\sigma^{*}(C)=C^{\prime}+\sum_{i=1}^{n} c_{i} E_{i}
$$

и

$$
\sigma^{*}(R)=E_{0}+\sum_{i=1}^{n} a_{i} E_{i}
$$

где $C^{\prime}, E_{0}$ и $E_{i}, 1 \leqslant i \leqslant n$, являются собственными прообразами в $U_{n}$ ростка $C$, слоя $R$ и исключительных дивизоров раздутий $\sigma_{i}, 1 \leqslant i \leqslant n$, соответственно. Используя эти обозначения, в качестве упомянутой выше константы $M$ можно взять любое $m$ такое, что

$$
m\left(\sum a_{i} E_{i}\right)-\left(\sum c_{i} E_{i}\right)
$$

является строго положительным дивизором, т. е. для всех $i$ выполнены неравенства $m a_{i}-c_{i}>0$. Действительно, пусть $s-$ особая точка кривой $\sigma^{-1}(C \cup R)_{\text {red }}$, принадлежащая $C^{\prime} \cap E_{i}$ для некоторого $i$. Выберем локальные координаты $\left(u_{i}, v_{i}\right)$ в окрестности точки $s$ так, что $u_{i}=0$ является уравнением $E_{i}$ и $v_{i}=0-$ уравнение кривой $C^{\prime}$. Имеем

$$
\sigma^{*}(u)=u_{i}^{a_{i}}, \quad \sigma^{*}(P(u, v))=u_{i}^{c_{i}} v_{i}
$$

с точностью до функции, не обращающейся в нуль в $s$, и

$$
\sigma^{*}(\bar{P}(u, v))=\left(v_{i}+u_{i}^{M a_{i}-c_{i}} \sum \sigma^{*}\left(v^{k-j} r_{j}(u)\right)\right) u_{i}^{c_{i}} .
$$

Следовательно, росток, заданный уравнением $\bar{P}(u, v)=0$, имеет то же самое разрешение особенностей, что и $(C, 0)$.

4.2. Локальная брэйд-монодромия над точкой (случай нескольких ростков). Пусть теперь $C \subset \mathbb{C}^{2}-$ аффинная приведенная алгебраическая кривая, заданная в координатах $(u, v)$ уравнением

$$
v^{m}+\sum_{i=1}^{m} q_{i}(u) v^{m-i}=0, \quad q_{i} \in \mathbb{C}[u] .
$$


Заметим, что любая аффинная алгебраическая кривая может быть задана такого типа уравнением после подходящей линейной замены координат.

Рассмотрим пересечение $C_{\varepsilon_{1}}=C \cap\left(D_{1}\left(\varepsilon_{1}\right) \times D_{2}\left(\varepsilon_{2}\right)\right), \varepsilon_{2}=\varepsilon_{1}^{-1}$. При условии $0<\varepsilon_{1} \ll 1$ проекция $\operatorname{pr}_{\mid \varepsilon_{\varepsilon_{1}}}: C_{\varepsilon_{1}} \rightarrow D_{1}=D_{1}\left(\varepsilon_{1}\right)$ является собственным отображением степени $m$ с единственным критическим значением $u=0$ (в противоположность ситуации в п. 4.1 здесь число критических точек может быть больше единицы). Допуская обычную вольность речи, назовем $C_{\varepsilon_{1}}$ ростком кривой $C$ над точкой 0 относительно pr. Как и в локальном случае (см. п. 4.1), зафиксируем точку $p \in \partial D_{1}$ и положим $D_{2, p}=\operatorname{pr}^{-1}(p), K=K(p)=\left\{v_{1}, \ldots, v_{m}\right\}=$ $D_{2, p} \cap C$. Снабжая $\partial D_{1}$ ориентацией против часовой стрелки, мы получаем над $\partial D_{1}$, с помощью проекции $\operatorname{pr}_{2}\left(C \cap \partial D_{1}\right)$, ориентированный след от движения $m$ точек в $D_{2}$ и, следовательно, косу $\tilde{b}_{\left(C_{\varepsilon_{1}}, 0\right)} \in \operatorname{Br}_{m}=\operatorname{Br}_{m}\left[D_{2}, K\right]$.

Пусть $\operatorname{pr}_{\mid C}^{-1}(0)=\left\{\left(0, v_{1}\right), \ldots,\left(0, v_{s}\right)\right\}$. Тогда росток $C_{\varepsilon_{1}}$ над точкой $u=0$ кривой $C$ распадается в несвязное объединение $C_{\varepsilon_{1}}=\bigsqcup_{i}^{s} C_{\varepsilon_{1}, i} \quad W$-подготовленных ростков $C_{\varepsilon_{1}, i}=\left(C,\left(0, v_{i}\right)\right)$ особенностей с кратностями $k_{i}, 1 \leqslant i \leqslant s$, $k_{1}+\cdots+k_{s}=m$, с центрами в точках $\left(0, v_{i}\right), 1 \leqslant i \leqslant s$. Пусть $k_{1}, \ldots, k_{t} \geqslant 2$ и $k_{t+1}=\cdots=k_{s}=1$. Далее нам нужно выбрать подходящий бидиск для каждой ветви ростка. Для этого выберем $\varepsilon_{3}>0$ и $\varepsilon_{1} \ll \varepsilon_{3}$ так, чтобы каждый росток $C_{\varepsilon_{1}, i}$ содержался в $D_{1} \times E_{i}$, где $E_{i}=\left\{\left|v-v_{i}\right|<\varepsilon_{3}\right\}$ и $E_{i} \cap$ $E_{j}=\varnothing$ для $i \neq j$. Положим $K_{i}=K_{i}(p)=\left\{v_{i, 1}, \ldots, v_{i, k_{i}}\right\}=D_{2, p} \cap C_{\varepsilon_{1}, i}$ и $E_{i, p}=\left(D_{1} \times E_{i}\right) \cap D_{2, p}$. Вложения $\left(E_{i}, K_{i}\right) \subset\left(D_{2, p}, K\right)$ определяют вложения $\eta_{i}: \operatorname{Br}_{k_{i}}\left[E_{i, p}, K_{i}\right] \subset \operatorname{Br}_{m}\left[D_{2, p}, K\right]$ так, что определена коса

$$
\tilde{b}_{\left(C_{\varepsilon_{1}}, 0\right)}=\prod_{i=1}^{s} \eta\left(b_{\left(C,\left(0, v_{i}\right)\right)}\right) .
$$

Как и в п 3.1, выберем в каждой группе $\mathrm{Br}_{k_{i}}\left[E_{i, p}, K_{i}\right]$ в качестве стандартных порождающих некоторые полуповороты. Объединение образов при отображениях $\eta_{i}$ этих порождающих может быть расширено до множества полуповоротов (обозначим их через $a_{1}, \ldots, a_{m-1}$ ), порождающих группу кос $\mathrm{Br}_{m}=$ $\operatorname{Br}_{m}\left[D_{2, p}, K\right]$ и удовлетворяющих соотношениям (3).

Таким образом, мы получаем косу

$$
b_{\left(C_{\varepsilon_{1}}, 0\right)}=\eta\left(b_{\left(C,\left(0, v_{1}\right)\right)}\right) \ldots \eta\left(b_{\left(C,\left(0, v_{t}\right)\right)}\right) \in \mathrm{Br}_{m}^{+}
$$

и называем ее стандартной формой брэйд-монодромии ростка кривой $C$ над точкой 0 .

Описанная выше конструкция зависит только от нумерации точек $p_{\mid C}^{-1}(0)=$ $\left\{\left(0, v_{1}^{0}\right), \ldots,\left(0, v_{s}^{0}\right)\right\}$ и расширения объединения образов образующих при отображениях $\eta_{i}$ до множества образующих группы $\mathrm{Br}_{m}=\mathrm{Br}_{m}\left[D_{2, p}, K\right]$. Поэтому определение стандартных форм брэйд-монодромии является однозначным с точностью до сопряюения.

В заключение этого пункта рассмотрим хорошо известное элементарное преобразование, заменяющее росток кривой над точкой на росток особенности. Именно, с ростком кривой $C$ над точкой $u=0$, заданным уравнением (16), 
можно связать другой росток $\bar{C}_{\varepsilon_{1}}$, заданный уравнением

$$
v^{m}+\sum_{i=1}^{m} u^{i} q_{i}(u) v^{m-i}=0 .
$$

Он имеет уже только одну точку над точкой $u=0$, и мы назовем его ассоциированной особенностъю кривой $C$ над точкой $u=0$.

Геометрический смысл ассоциированной особенности состоит в следующем. Мы можем рассмотреть $\mathbb{C}^{2}$ с координатами $(u, v)$ как аффинную карту некоторой линейчатой поверхности $\mathrm{pr}: F_{N} \rightarrow \mathbb{P}^{1}, N>0$, не пересекающуюся с исключительным сечением $E_{N}$ поверхности $F_{N}$, а $C$ - как алгебраическую кривую, содержащуюся в этой карте поверхности $F_{N}$. Сделаем элементарное преобразование $\tau: F_{N} \rightarrow F_{N+1}$ с центром в точке пересечения $E_{N}$ со слоем $R_{0}$ над точкой $u=0$. Тогда уравнение $(17)$ есть уравнение образа $\tau(C) \subset F_{N+1}$ в соответствующей карте поверхности $F_{N+1}$. Ассоциированная особенность определяет однозначно росток кривой $C$ : чтобы получить его, достаточно проделать обратное преобразование $\tau^{-1}$.

Следующее утверждение связывает между собой стандартную форму брэйдмонодромии ростка кривой с брэйд-монодромией ассоциированной с ней особенности.

Лемма 4.2 [20]. Пусть $\left(C_{\varepsilon_{1}}, 0\right)$ - росток алгебраической кривой степени $m$ над точкой $u=0$ u $\left(\bar{C}_{\varepsilon_{1}}, o\right)$ - росток ассоциированной с ней особенности. Тогда

$$
b_{\left(\bar{C}_{\varepsilon_{1}}, 0\right)}=b_{\left(C_{\varepsilon_{1}}, o\right)} \Delta_{m}^{2} .
$$

Так как росток кривой определяется своей ассоциированной особенностью, то из этого утверждения вытекает, что ростки $\left(C_{1, \varepsilon_{1}}, 0\right)$ и $\left(C_{2, \varepsilon_{1}}, 0\right)$ топологически эквивалентны тогда и только тогда, когда $b_{\left(C_{1, \varepsilon_{1}}, 0\right)}=b_{\left(C_{2, \varepsilon_{1}}, 0\right)}$ и, следовательно, топологический тип ростка $\left(C_{\varepsilon_{1}}, 0\right)$ кривой $C$ над точкой $u=0$ однозначно определяется своей монодромией $b_{\left(\varepsilon_{\varepsilon_{1}}, 0\right)}$.

Рассмотрим все стандартные формы брэйд-монодромий всех ростков над точкой 0 всех алгебраических кривых степени $m$. Обозначим через $P$ объединение их орбит при действии сопряжением группы $\mathrm{Br}_{m}$ и положим $\mathscr{P}=$ $S\left(\mathrm{Br}_{m}, P\right)$. Заметим, что $A_{k} \subset P$, где $A_{k}$ было определено в п. 3.2 (в частности, элементы, принадлежащие $A_{0}$, соответствуют простым точкам касания кривой $C$ и слоя $u=0$, элементы, принадлежащие $A_{1}$, соответствуют ноудам, а элементы, принадлежащие $A_{2}$, соответствуют обыкновенным каспам).

4.3. Разложение на множители брэйд-монодромии над диском. Как и в п. 4.2, рассмотрим многочлен $P(u, v)=v^{m}+\sum_{i=1}^{m} q_{i}(u) v^{m-i} \in \mathbb{C}[u, v]$, не имеющий кратных множителей, и множество его нулей - кривую $C$ в $\mathbb{C}^{2}$, заданную уравнением $P(u, v)=0$.

Выберем в группе $\pi_{1}\left(\bar{D}_{1}(r) \backslash\left\{u_{1}, \ldots, u_{n}\right\}, u_{0}\right)$ хороший геометрический базис $\left\{\gamma_{1}, \ldots, \gamma_{n}\right\}$. Как и в п. 4.2, каждую петлю $\gamma_{i}, 1 \leqslant i \leqslant n$, можно поднять в $C$ и получить $m$ непересекающихся друг с другом путей, начинающихся и заканчивающихся в точках из множества $K\left(p_{0}\right)$, и тем самым получить элемент $b_{i}=b\left(\gamma_{i}\right) \in \mathrm{Br}_{m}=\mathrm{Br}_{m}\left[D_{2}, K\left(p_{0}\right)\right]$, где $D_{2}$ - диск большого радиуса в $v$-плоскости. 
Свободная группа $\pi_{1}\left(\bar{D}_{1}(r) \backslash\left\{u_{1}, \ldots, u_{n}\right\}, u_{0}\right)$ свободно порождается элементами $\gamma_{i}$ и поэтому сопоставление $\gamma_{i} \mapsto b_{i} \in \mathrm{Br}_{m}$ задает гомоморфизм монодромии

$$
\beta: \pi_{1}\left(\bar{D}_{1}(r) \backslash\left\{u_{1}, \ldots, u_{n}\right\}, u_{0}\right) \rightarrow \mathrm{Br}_{m}
$$

Имеем $\beta\left(\partial D_{1}(r)\right)=b_{1} \ldots b_{n}$.

Для каждого $1 \leqslant i \leqslant n$ в классе сопряженности элемента $b_{i}$ содержится стандартная форма брэйд-монодромии ростка кривой $C$ над точкой $u_{i}$, т. е. $b_{i}=$ $b\left(\gamma_{i}\right) \in P \subset \mathrm{Br}_{m}$. Сопоставим тройке $\left(D_{1}(r), P(u, v)\right.$, pr) набор $\left\{b_{1}, \ldots, b_{n}\right\} \in P^{n}$. Этот набор зависит от выбора хорошего геометрического базиса. Если выбран другой хороший геометрический базис $\left\{\gamma_{1}^{\prime}, \ldots, \gamma_{n}^{\prime}\right\}$ фундаментальной группы $\pi_{1}\left(\bar{D}_{1}(r) \backslash\left\{u_{1}, \ldots, u_{n}\right\}, u_{0}\right)$, то он определяет другой набор $\left\{b_{1}^{\prime}, \ldots, b_{n}^{\prime}\right\} \in P^{n}$, в котором $b_{i}^{\prime}=\beta\left(\gamma_{i}^{\prime}\right)$. Рассмотрим стандартное действие группы $\mathrm{Br}_{n}$ на свободной группе $\left\langle\gamma_{1}, \ldots, \gamma_{n}\right\rangle$. Так как $\gamma_{1} \ldots \gamma_{n}=\gamma_{1}^{\prime} \ldots \gamma_{n}^{\prime}=\partial D_{1}(r)$, то найдется такой элемент $\bar{b} \in \mathrm{Br}_{n}$, что $\left(\gamma_{i}\right) \bar{b}=\gamma_{i}^{\prime}$ для всех $i$. Согласно формулам (4) имеем $\beta\left(\left(\gamma_{j}\right) \bar{a}_{i}\right)=\beta\left(\left(\gamma_{j}\right) \bar{a}_{i}^{-1}\right)=b_{j}$, если $j \neq i, i+1$, и

$$
\begin{aligned}
\beta\left(\left(\gamma_{i}\right) \bar{a}_{i}\right) & =b_{i} b_{i+1} b_{i}^{-1}, \\
\beta\left(\left(\gamma_{i+1}\right) \bar{a}_{i}\right) & =b_{i},
\end{aligned}
$$

а также

$$
\begin{aligned}
\beta\left(\left(\gamma_{i}\right) \bar{a}_{i}^{-1}\right) & =b_{i+1}, \\
\beta\left(\left(\gamma_{i+1}\right) \bar{a}_{i}^{-1}\right) & =b_{i+1}^{-1} b_{i} b_{i+1},
\end{aligned}
$$

где $\bar{a}_{i}$ - стандартные порождающие группы $\mathrm{Br}_{n}$, стандартно действующие на свободной группе $\left\langle\gamma_{1}, \ldots, \gamma_{n}\right\rangle$, т. е. набор $\left\{\beta\left(\left(\gamma_{1}\right) \bar{a}_{i}^{ \pm 1}\right), \ldots, \beta\left(\left(\gamma_{n}\right) \bar{a}_{i}^{ \pm 1}\right)\right\}$ для каждого $i$ получается из набора $\left\{b_{1}, \ldots, b_{n}\right\}$ с помощью преобразований Гурвица. Отсюда легко получить, что наборы $\left\{b_{1}, \ldots, b_{n}\right\}$ и $\left\{b_{1}^{\prime}, \ldots, b_{n}^{\prime}\right\}$, соответствующие хорошим геометрическим базисам $\left\{\gamma_{1}, \ldots, \gamma_{n}\right\}$ и $\left\{\gamma_{1}^{\prime}, \ldots, \gamma_{n}^{\prime}\right\}$, являются Гурвиц-эквивалентными, и поэтому тройке $\left(D_{1}(r), P(u, v), \mathrm{pr}\right)$ естественно сопоставлять разложение на множители

$$
\left\{b_{1}, \ldots, b_{n}\right\} \in P^{n} \subset\left(\mathrm{Br}_{m}\right)^{n}
$$

элемента $\beta\left(\partial D_{1}(r)\right) \in \mathrm{Br}_{m}$ с множителями из $P$, т. е. сопоставлять элемент

$$
\operatorname{bmf}\left(C, D_{1}(r)\right)=b_{1} \cdot \ldots \cdot b_{n} \in \mathscr{P}
$$

который называется разложением на множители брэйд-монодромии многочлена $P(u, v)$ (или, что то же самое, кривой $C$ ) над диском $D_{1}(r)$. Если $P(u, v)$ не имеет критических значений вне $D_{1}(r)$, то разложение на множители брэйдмонодромии будем обозначать просто $\operatorname{bmf}(P(u, v))$ (соответственно $\operatorname{bmf}(C))$.

ПреДЛОЖЕНИЕ 4.1 [9]. Пусть $C \subset F_{N}$ - алгебраическая кривая такая, что $C \cap E_{N}=\varnothing$ и ограничение $\operatorname{pr}_{\mid C}$ проекиии $\mathrm{pr}$ на $C$ является накрытием проективной прямой $\mathbb{C P}^{1}$ степени $\operatorname{deg} \operatorname{pr}_{\mid C}=m$. Тогда $\alpha(\operatorname{bmf}(C))=\Delta_{m}^{2 N}$. 
ДокАЗАТЕЛЬство. Пусть все критические значения кривой $C$ лежат в некотором диске $D_{1}(r)$. Для некоторого $R \gg r$ существует алгебраическая деформация $C_{t}$ кривой $C$ в $F_{N}$ такая, что, во-первых, все критические точки кривых $C_{t}$ относительно проекции $\mathrm{pr}$ лежат в $\operatorname{pr}^{-1}\left(D_{1}(R)\right)$, а во-вторых, $C_{1}$ в карте $\mathbb{C}_{1}^{2}$ задается уравнением $v_{1}^{m}=u_{1}^{m N}$. Тогда, во-первых, $\operatorname{bmf}\left(C_{t}\right)$ не меняется при изменении $t$, а во-вторых, элемент $\alpha\left(\operatorname{bmf}\left(C_{1}\right)\right)$ совпадает с монодромией ростка $(C, o)$ алгебраической кривой, заданной уравнением $v^{m}=u^{m N}$, относительно проекции pr. Согласно лемме 4.1 эта монодромия равна $\left(a_{1} \ldots a_{m-1}\right)^{m N}=\Delta_{m}^{2 N}$. Предложение 4.1 доказано.

Конкретный вид разложения на множители брэйд-монодромии $\operatorname{bmf}(C)$ зависит от выбора стандартных порождающих (полуповоротов) $a_{1}, \ldots, a_{m-1}$ группы $\operatorname{Br}_{m}\left[D_{2}, K\left(p_{0}\right)\right]$. При другом выборе стандартных порождающих $a_{1}^{\prime}, \ldots, a_{m-1}^{\prime}$ найдется такой элемент $b \in \operatorname{Br}_{m}\left[D_{2}, K\left(p_{0}\right)\right]$, что $a_{1}^{\prime}=b a_{i} b^{-1}$ для всех $i$. Поэтому разложение на множители брэйд-монодромии $\operatorname{bmf}(C)$, выраженное через порождающие $a_{1}^{\prime}, \ldots, a_{m-1}^{\prime}$, равно $\lambda(b)(\operatorname{bmf}(C))$. Другими словами, инвариантно определен только тип разложения на множители брэйд-монодромии многочлена $P(u, v)$ над $D_{1}(r)$ (соответственно кривой $C$, заданной уравнением $P(u, v)=0)$, который будем обозначать через $\operatorname{bmt}\left(P(u, v), D_{1}(r)\right)$ (соответственно $\left.\operatorname{bmt}\left(C, D_{1}(r)\right)\right)$. Если $P(u, v)$ не имеет критических значений вне $D_{1}(r)$, то тип разложения на множители брэйд-монодромии будем обозначать просто $\operatorname{bmt}(P(u, v))$ (соответственно $\operatorname{bmt}(C))$.

Отметим, что все понятия, введенные в этом пункте, дословно переносятся на любую замкнутую область в $\mathbb{C}$, диффеоморфную замкнутому диску.

Tеорема 4.1 [20], [36]. Для любого элемента $s=b_{1} \cdot \ldots \cdot b_{n} \in \mathscr{P} \subset S_{\mathrm{Br}_{m}}$ существуют натуральное число $N$ и алгебрачческая кривая $C \subset F_{N}, C \cap$ $E_{N}=\varnothing$, такая, что $\operatorname{bmf}\left(C, D_{1}(1)\right)=s u \operatorname{bmf}(C)=s \cdot s^{\prime}$, где $s^{\prime} \in \mathscr{A}_{0}$.

Идея доказательства этой теоремы состоит в следующем. Вначале строится алгебраическая кривая $\widetilde{C} \subset F_{N_{1}}$ с достаточно большим $N_{1}$, разложение брэйд-монодромии которой над некоторым диском $D_{1}(r) \operatorname{paвно~} \operatorname{bmf}\left(\widetilde{C}, D_{1}(r)\right)=$ $\bar{b}_{1} \cdot \ldots \cdot \bar{b}_{n}$, где каждый элемент $\bar{b}_{i}$ сопряжен элементу $b_{i}$, a вне $\operatorname{pr}^{-1}\left(D_{1}(r)\right)$ кривая $\widetilde{C}$ имеет только простейшие критические точки (типа $A_{0}$ ). Из леммы 3.3 следует, что найдется такой элемент $s_{1} \in \nu\left(\mathrm{Br}_{m}^{+}\right) \subset S_{A_{0}}$, что $\alpha\left(s \cdot s_{1}\right)=\Delta_{m}^{2 N_{2}}$ при некотором $N_{2} \gg 0$. Переходом от $F_{N_{1}}$ к $F_{N_{1}+1}$ с помощью элементарного преобразования (см. п. 4.1) и сглаживания особой точки типа $v^{m}=u^{m}$ мы можем увеличивать $N_{1}$, не меняя $\operatorname{bmf}\left(\widetilde{C}, D_{1}(r)\right)$. Поэтому мы можем считать, что $N_{1}=N_{2}, \alpha(\operatorname{bmf}(\widetilde{C}))=\Delta_{m}^{2 N_{2}}$, и после этого применить теорему 3.1, согласно которой найдется такое $M$, что $s \cdot s_{1} \cdot\left(\delta_{m}^{2}\right)^{M}=\operatorname{bmf}(\widetilde{C}) \cdot\left(\delta_{m}^{2}\right)^{M}$. Снова, с помощью перехода от $F_{N_{1}}$ к $F_{N_{1}+M}$ с помощью элементарных преобразований, сглаживая особые точки типа $v^{m}=u^{m}$, получим кривую $\bar{C} \subset F_{N_{1}+M}$, которая имеет разложение на множители брэйд-монодромии, равное $s \cdot s_{1} \cdot\left(\delta_{m}^{2}\right)^{M}$. Следовательно, найдется открытое множество $U \subset \mathbb{C P}^{1}$, голоморфно диффеоморфное диску $D_{1}(1)$, такое, что $\operatorname{bmf}(\bar{C}, U)=s$, и тем самым мы находим кривую $\bar{C} \subset D_{1}(1) \times \mathbb{C}^{1}$, заданную в этом произведении уравнением $v^{m}+\sum_{i=0}^{m-1} c_{i}(u) v^{i}$, $c_{i}(u) \in \mathbb{C}\{u\}$, разложение на множители брэйд-монодромии которой равно 
$\operatorname{bmf}\left(\bar{C}, D_{1}(1)\right)=s$. Наконец, аппроксимируя степенные ряды $c_{i}(u)$ многочленами, находим требуемую кривую $C$.

УТВЕРЖДЕНИЕ 4.1. Пусть $C \subset F_{N}$ - неособая алгебраическая кривая такая, что $C \cap E_{N}=\varnothing$ и ограничение $\operatorname{pr}_{\mid C}$ проекции $\mathrm{pr}$ на $C$ является общим накрытием проективной прямой $\mathbb{C P}^{1}$ степени $\operatorname{deg} \operatorname{pr}_{\mid C}=m$, m.е. $\#\left\{\operatorname{pr}^{-1}(p)\right\} \geqslant$ $m-1$ для каждой точки $p \in \mathbb{C P}^{1}$. Тогда $\operatorname{bmf}(C)=\left(\delta_{m}^{2}\right)^{N}$.

ДокАзАтЕльство. Вначале доказываем это утверждение для $N=1$ индукцией по $m$, добавляя к кривой степени $m-1$ в $\mathbb{C P}^{2}$ общую прямую $L$ и сглаживая особые точки кривой $C \cup L$ (см. [6]). Затем, переходя от $F_{N}$ к $F_{N+1}$ с помощью элементарного преобразования (см. п. 4.1) и сглаживая особую точку типа $v^{m}=u^{m}$, получаем общую формулу.

Пусть $a_{1}, \ldots, a_{m-1}-$ стандартные порождающие группы кос $\mathrm{Br}_{m}$. Положим $z_{k, l}=\left(a_{l-1} \ldots a_{k+1}\right) a_{k}\left(a_{l-1} \ldots a_{k+1}\right)^{-1}$ для $k<l$ и рассмотрим элемент

$$
\tilde{\delta}_{m}^{2}=\prod_{l=m}^{2} \prod_{k=1}^{l-1} z_{k, l}^{2} \in S_{A_{1}}
$$

(через $\prod_{l=m}^{2}$ обозначено произведение слева направо упорядоченной по убыванию $l$ последовательности элементов $z_{k, l}^{2}$ ). Известно (см., например, [9]), что $\alpha\left(\tilde{\delta}_{m}^{2}\right)=\Delta_{m}^{2}$. Более того,

$$
\tilde{\delta}_{m}^{2}=\prod_{k=1}^{m-1} z_{k, m}^{2} \cdot \tilde{\delta}_{m-1}^{2}
$$

(здесь мы отождествляем группу $\mathrm{Br}_{m-1}$ с подгруппой группы $\mathrm{Br}_{m}$, порожденной элементами $\left.a_{1}, \ldots, a_{m-2}\right)$.

Лемма 4.3. Имеют место следующие равенства:

(i) $a_{k} z_{j, k}^{2} a_{k}^{-1}=z_{j, k+1}^{2}$;

(ii) $a_{k} z_{k, m}^{2} a_{k}^{-1}=z_{k+1, m}^{2}$;

(iii) $a_{k} z_{j, r}^{2} a_{k}^{-1}=z_{j, r}^{2}$, если либо $r<k$, либо $j<k$, либо $j>k+1$;

(iv) $a_{k} z_{k+1, m}^{2} a_{k}^{-1}=z_{k+1, m}^{-2} z_{k, m}^{2} z_{k+1, m}^{2}$.

ДокАЗАТЕЛЬство следует из соотношений (3) и определения элементов $z_{k, j}^{2}$.

ПредлоЖениЕ 4.2. Элемент $\tilde{\delta}_{m}^{2} \in S_{A_{1}}$ является неподвижным при действии сопряжением группь $\mathrm{Br}_{m}$.

ДоКАЗАТЕЛЬСТво. Индукцией по $m$, с использованием леммы 4.3, непосредственно проверяется, что $\lambda\left(a_{i}\right)\left(\tilde{\delta}_{m}^{2}\right)=\tilde{\delta}_{m}^{2}$ для $i=1, \ldots, m-1$.

УтВеРЖДЕНИЕ 4.2. Пусть $C=\bigcup_{j=1}^{m} L_{j} \subset F_{N}$ - объединение алгебраических сечений $L_{j}$ проекции $\mathrm{pr}$, находящихся в общем положении (т.е. если два из этих сечений пересекаются в некоторой точке $p \in F_{N}$, то они пересекаются трансверсально и через эту точку не проходят другие сечения), такое, что $C \cap E_{N}=\varnothing$. Тогда $\operatorname{bmf}(C)=\left(\tilde{\delta}_{m}^{2}\right)^{N}$.

ДокАзАТЕЛЬСтво. Аналогично доказательству утверждения 4.1. 


\section{4. Разложение на множители брэйд-монодромии кривой Гурви-}

ца. Так как критические точки кривой Гурвица $\bar{H} \subset F_{N}, \operatorname{deg} \bar{H}=m$, не имеющей отрицательных ноудов, являются аналитическими, а вне критических точек кривая $\bar{H}$ локально представляет собой сечения проекции pr, то определение разложения на множители брэйд-монодромии над диском $D_{1}(r) \subset \mathbb{C P}^{1}$, данное в предыдущем пункте, дословно переносится с алгебраического случая на случай кривых Гурвица.

Это определение также переносится на случай кривых Гурвица с отрицательными ноудами, надо только заменить полное множество $P$ монодромий ростков алгебраических кривых на полное множество $\widetilde{P}=P \cup A_{-3}$. В этом случае разложение брэйд-монодромии на множители $\operatorname{bmf}\left(\bar{H}, D_{1}(r)\right)$ кривой Гурвица $\bar{H}$ с отрицательными ноудами над диском $D_{1}(r) \subset \mathbb{C P}^{1}$ принадлежит полугруппе $\widetilde{\mathscr{P}}=S\left(\mathrm{Br}_{m}, \widetilde{P}\right)$.

ПРЕДЛОЖЕНИЕ 4.3. Пусть $\bar{H} \subset F_{N}-$ кривая Гурвица (возможсно, с отрииательными ноудами), $\operatorname{deg} \bar{H}=m$. Тогда $\alpha(\operatorname{bmf}(\bar{H}))=\Delta^{2 N}$.

ДокАЗАтЕЛьство. Пусть все критические значения кривой $\bar{H}$ лежат в некотором диске $D_{1}(r)$. Выберем некоторое $R \gg r$. Над окрестностью $U=\mathbb{C P}^{1} \backslash \bar{D}_{1}(R)$ точки $\infty$ пересечение $\bar{H} \cap \mathrm{pr}^{-1}(U)$ является несвязным объединением $m$ сечений проекции pr. Следовательно, существует гладкая $H$-изотопия $h_{t}$ (см. определение $H$-изотопии в следующем параграфе) многообразия $F_{N}$ такая, что, во-первых, изотопия $h_{t}$ является тождественной в точках, лежащих в $\operatorname{pr}^{-1}\left(D_{1}(R)\right)$, а во-вторых, $h_{1}(\bar{H})$ совпадает над $U$ с $m$ сечениями, заданными уравнением $v_{2}^{m}-1=0$ в пересечении $\mathbb{C}_{2}^{2} \cap \operatorname{pr}^{-1}(U)$, и предложение 4.3 следует из предложения 4.1.

Tеорема 4.2 [20]. Для любого элемента $s \in \widetilde{\mathscr{P}} \subset S_{\mathrm{Br}_{m}}$ такого, что $\alpha(s)=$ $\Delta_{m}^{2 N}$, где $N \in \mathbb{N}$, существует кривая Гурвица $\bar{H} \subset F_{N}$ степени $m$ такая, ито $\operatorname{bmf}(\bar{H})=s$. Если $s \in \mathscr{P} \subset S_{\mathrm{Br}_{m}}$, то найдется полуалгебрачческая кривая Гурвица $\bar{H} \subset F_{N}$ такая, ито $\operatorname{bmf}(\bar{H})=s$.

ДокаЗательство. Пусть $s=s_{1} \cdot b_{1} \cdot \ldots \cdot b_{k}$, где $s_{1} \in \mathscr{P}$ и $b_{1}, \ldots, b_{k} \in A_{-3}$. Согласно теореме 4.1 существует алгебраическая кривая $C \subset \mathbb{C}_{1}^{2}$ такая, что $\operatorname{bmf}\left(C, D_{1,1}(1)\right)=s_{1}$. Выберем $k$ дисков $D_{1, j}(1)=\{u \in \mathbb{C}|| u+3(j-1) \mid<1\}$, $j=2, \ldots, k+1$, в $\mathbb{C}_{1}^{1}=\operatorname{pr}\left(\mathbb{C}_{1}^{2}\right)$ с центрами в точках $p_{2}=\{u=-3\}, \ldots, p_{k+1}=$ $\{u=-3 k\}$ и положим $p_{1}=\{u=0\}, p_{0}=\{u=-4 k i\}$, где $i=\sqrt{-1}$. Пусть $c_{j}=$ $\partial \bar{D}_{1, j}(1)$ - граница диска $\bar{D}_{1, j}(1)$. Обозначим через $\bar{D}_{1}=\bar{D}_{1}(4 k)$ замкнутый диск радиуса $4 k$ с центром в начале координат, $p_{0} \in \partial \bar{D}_{1}$, и выберем геометрический базис $\gamma_{1}, \ldots, \gamma_{k+1}$ группы $\pi_{1}\left(\bar{D}_{1} \backslash\left\{p_{1}, p_{2}, \ldots, p_{k+1}\right\}, p_{0}\right)$, где $\gamma_{j}=l_{j} c_{j} l_{j}^{-1}$ и $l_{j}$ - отрезок прямой, соединяющий точку $p_{0}$ с точкой $p_{0, j}=\{u=-3(j-1)-i\}$.

Фиксируем конечное множество $K=\left\{v_{1}, v_{2}, \ldots, v_{m}\right\}=\operatorname{pr}^{-1}\left(p_{0,1}\right) \cap C$ и выбираем в $\operatorname{Br}_{m}\left[D_{2}, K\left(p_{0,1}\right)\right]$ стандартные порождающие (положительные полуповороты) $a_{1}, \ldots, a_{m-1}$ группы $\mathrm{Br}_{m}\left[D_{2}, K\left(p_{0,1}\right)\right]$, записанное в которых брэйд-монодромное разложение $\operatorname{bmf}\left(C, D_{1,1}(1)\right)$ равно $s_{1}$, где $D_{2}$ - диск большого радиуса в $v$-плоскости.

Вначале, чтобы построить нужную нам кривую Гурвица $\bar{H}$, строим "кусок" этой кривой над дисками $D_{1, j}(1):$ над диском $D_{1,1}(1)$ кривая $\bar{H}$ совпадает с кривой $C$, а над дисками $D_{1, j}(1), j=2, \ldots, k+1$, кривая $\bar{H}$ состоит из несвязного 
объединения $m-2$ сечений $v=q_{t}>1, t=3, \ldots, m$, и кривой, заданной уравнением $(v+u-3(j-1))(v-\bar{u}+3(j-1))=0$. Затем продолжаем построение кривой $\bar{H}$ над объединением $\left(\bigcup \bar{D}_{1, j}(1)\right) \cup\left(\bigcup l_{j}\right)$, добавляя над каждым путем $l_{j}$ геометрическую косу, соединяющую точки из $K\left(p_{0}\right)=K\left(p_{0,1}\right)$ с точками, лежащими в $\operatorname{pr}^{-1}\left(p_{0, j}\right) \cap \bar{H}$, так, чтобы над каждой петлей $\gamma_{j}$ построенная коса представляла элемент $b_{j} \in \mathrm{Br}_{m}\left[D_{2}, K\left(p_{0}\right)\right]$ при $j \geqslant 2$, а коса над $l_{1}$ состояла из постоянных сечений. Очевидно, что это построение может быть продолжено на некоторую замкнутую окрестность $U$ множества $\left(\bigcup \bar{D}_{1, j}(1)\right) \cup\left(\bigcup l_{j}\right) \backslash\left\{p_{0}\right\}$, диффеоморфного замкнутому диску. Выберем диск $D_{1}(R) \subset \mathbb{C}^{1}$ большого радиуса, содержащий $U$, и соединим точку $p_{0} \in \partial U$ с некоторой точкой $p \in \partial \bar{D}_{1}(R)$ гладким путем $l:[0,1] \rightarrow \bar{D}_{1}(R) \backslash U$.

Определим кривую $\bar{H}$ над дополнением к $D_{1}(R)$ в $\mathbb{C P}^{1}$ как объединение $m$ алгебраических кривых, заданных в $\mathbb{C}_{1}^{2}$ уравнением $v^{m}-u^{m N}=0$. Очевидно, что множество $\bar{D}_{1}(R) \backslash\left(U \cup \partial \bar{D}_{1}(R) \cup l\right)$ диффеоморфно диску и существует гладкая изотопия $h_{t}$ комплексной прямой $\mathbb{C}^{1}$ такая, что $h_{t}(l(0))=l(t), h_{1}(\partial U)=\partial D_{1}(R)$ и $h_{t_{1}}(\partial U) \cap h_{t_{2}}(\partial U)=\varnothing$ при $t_{1} \neq t_{2}$. По построению коса, определяемая кривой Гурвица $\bar{H}$ над $\partial U$, равна $\Delta_{m}^{2 N}$. Следовательно, изотопия $h_{t}$ может быть поднята до гладкой изотопии $\tilde{h}_{t}$ комплексной плоскости $\mathbb{C}^{2}$, соединяющей построенные косы (равные $\Delta_{m}^{2 N}$ ) над $\partial U$ и $\partial \bar{D}_{1}(R)$. Эта изотопия продолжает построенную уже часть кривой Гурвица $\bar{H}$ до кривой Гурвица, определенной над всей комплексной прямой $\mathbb{C P}^{1}$. По построению брэйд-монодромное разложение на множители, ассоциированное с кривой $\bar{H}$, совпадает с $s$. Если $k=0$, то построенная кривая $\bar{H}$ является почти алгебраической кривой Гурвица. Теорема 4.2 доказана.

4.5. Брэйд-монодромное разложение на множители псевдоголоморфной кривой в $\mathbb{C P}^{2}$. Пусть $\omega$ - форма Фубини-Штуди на комплексной проективной плоскости $\mathbb{C P}^{2}$, и пусть $J-\omega$-ручная почти комплексная структура на $\mathbb{C P}^{2}$.

В [37] было показано, что для произвольной ручной почти комплексной структуры $J$ на $\mathbb{C P}^{2}$ можно определить $J$-прямые как псевдоголоморфные сферы $L$, представляющие порождающий элемент группы $H_{2}\left(\mathbb{C P}^{2}, \mathbb{Z}\right)$, причем геометрия $J$-прямых, до некоторой степени, является такой же, как и стандартная проективная геометрия. Например, существует единственная $J$-прямая, проходящая через каждые две точки в $\mathbb{C P}^{2}$, и эта $J$-прямая гладко зависит от точек и от почти комплексных структур $J$. Таким образом, для каждой точки $p \in \mathbb{C P}^{2}$ имеем пучок $J$-прямых, проходящих через точку $p$, параметризованный точками двумерной сферы. Более того, все такие пучки (для всех почти комплексных структур $J$ ) симплектически изотопны. Используя теорему Мозера, можно показать, что если удалить одну из $J$-прямых, то дополнение будет послойно симплектоморфно аффинной плоскости $\mathbb{C}^{2}$.

Для заданной $J$-голоморфной кривой $\bar{H} \in \mathbb{C P}^{2}$, принадлежащей классу $m[L] \in H_{2}\left(\mathbb{C P}^{2}, \mathbb{Z}\right)$ при некотором $m \in \mathbb{N}$, и фиксированной точки $p_{\infty} \in \mathbb{C P}^{2}$, $p_{\infty} \notin \bar{H}$, почти все $J$-голоморфные прямые $R$, принадлежащие пучку $J$-прямых $P_{p_{\infty}}$, проходящих через точку $p_{\infty}$ (кроме, скажем, $J$-прямых $R_{1}, \ldots, R_{n}$ ), пересекают кривую $\bar{H}$ ровно в $m$ точках. Выберем одну из таких прямых и обозначим ее через $R_{\infty}$. Тогда $\mathbb{C P}^{2} \backslash R_{\infty}$ диффеоморфно $\mathbb{C}^{2}$ и пучок $J$-прямых $P_{p_{\infty}}$ 
определяет тривиальное $C^{\infty}$-расслоение $\mathrm{pr}: \mathbb{C P}^{2} \backslash R_{\infty} \rightarrow \mathbb{R}^{2} \simeq \mathbb{C}$, слоями которого являются дополнения к точке $p_{\infty}$ в $J$-голоморфных прямых $R$, принадлежащих пучку $P_{p_{\infty}}, R \backslash p_{\infty} \simeq \mathbb{C}$. Обозначим $p_{j}=\operatorname{pr}\left(R_{j}\right) \in \mathbb{R}^{2}$, и пусть $p_{0}=\operatorname{pr}\left(R_{0}\right)$, где $R_{0}$ - некоторый слой проекции $\mathrm{pr}, p_{0} \neq p_{j}$ для $j=1, \ldots, n$. Выберем в $\pi_{1}\left(\mathbb{R}^{2} \backslash\left\{p_{1}, \ldots, p_{n}\right\}, p_{0}\right)$ хороший геометрический базис $\gamma_{1}, \ldots, \gamma_{n}$. Как и в случае кривых Гурвица, подъем петель $\gamma_{j}$ на псевдоголоморфную кривую $\bar{H}$ определяет элементы $b_{j} \in \mathrm{Br}_{m}[D, K]$, где $K=\bar{H} \cap \operatorname{pr}^{-1}(0)$ и $D-$ диск в $\mathrm{pr}^{-1}(0)$ достаточно большого радиуса, и, тем самым, определяет элемент $s \in S_{\mathrm{Br}_{m}}$, который называется разложением на множители брэйд-монодромии псевдоголоморфной кривой $\bar{H}$ относительно пучка $J$-прямых $P_{p_{\infty}}$ и обозначается $\operatorname{bmf}\left(\bar{H}, P_{p_{\infty}}\right)$. Так как особые точки псевдоголоморфных кривых на почти комплексных четырехмерных многообразиях эквивалентны с точностью до $C^{1}$-замены координат особым точкам обычных комплексных кривых на комплексных поверхностях, то $\operatorname{bmf}\left(\bar{H}, P_{p_{\infty}}\right) \in \mathscr{P}$.

4.6. Гомотопический тип дополнения к аффинной кривой Гурвица. Как и выше, пусть $H \subset \mathbb{C}^{2}-$ аффинная "часть" кривой Гурвица $\bar{H} \subset F_{N}$ степени $m$ (возможно, с отрицательными ноудами), $\operatorname{bmf}(H)=b_{1} \cdot \ldots \cdot b_{n}-$ разложение на множители брэйд-монодромии кривой $\bar{H}, b_{i}=g_{i}^{-1} \bar{b}_{i} g_{i}$, где $\bar{b}_{i} \in \mathrm{Br}_{k_{i}, 0} \subset \mathrm{Br}_{m}$ - стандартные формы брэйд-монодромии критических точек кривой $H$.

Разложение $\operatorname{bmf}(H)$ на множители определяет гомотопический тип пространства $\mathbb{C}^{2} \backslash H$.

ТеОрема 4.3. Пространство $\mathbb{C}^{2} \backslash H$ гомотопически эквивалентно двумерному комплексу, ассоциированному с копредставлением

$$
\left\langle x_{1}, \ldots, x_{m} \mid\left(x_{i}\right) g_{j}=\left(x_{i}\right) \bar{b}_{j} g_{j}, i=1, \ldots, m, j=1, \ldots, n\right\rangle
$$

групnъь $\pi_{1}\left(\mathbb{C}^{2} \backslash H\right)$.

Доказательство этой теоремы, приведенное в [38] в случае, когда $\bar{H}$ является алгебраической кривой в $\mathbb{C P}^{2}$, может быть дословно перенесено на случай кривых Гурвица $\bar{H} \subset F_{N}$.

Так, например, если $\bar{H}$ является неособой алгебраической кривой в $\mathbb{C P}^{2}$ степени $m$, то пространство $\mathbb{C}^{2} \backslash H$ гомотопически эквивалентно букету $S^{1} \vee$ $\left(\bigvee_{i=1}^{(m-1)^{2}} S_{i}^{2}\right)$ окружности и $(m-1)^{2}$ двумерных сфер.

\section{§ 5. Деформации и $H$-изотопии кривых Гурвица}

5.1. Эквисингулярные деформации кривых Гурвица. Семейство кривых Гурвица $\bar{H}_{t}$ (возможно, с отрицательными ноудами), $t \in[0, \varepsilon]$, заданных уравнениями

$$
v_{i}^{m}+\sum_{j=0}^{m-1} c_{i, j}\left(u_{i}, t\right) v_{i}^{j}=0
$$

в картах $\mathbb{C}_{i}^{2}$, непрерывно зависящими от $t$, такими, что

1) при каждом $t \in[0, \varepsilon]$ уравнения (18) являются уравнениями некоторой кривой Гурвица $\bar{H}_{t}$; 
2) множество Crit $\bar{H}_{t}$ является несвязным объединением непрерывных путей (траекторий критических точек),

называется эквисингулярной деформацией кривой Гурвица $\bar{H}_{0}$ и - эквисингулярной $H$-деформащией, если для всех $t$ и каждой точки $p_{t} \in \operatorname{Crit} \bar{H}_{t}$ число $\#\left(\bar{H}_{t} \cap R_{\operatorname{pr}\left(p_{t}\right)}\right)$ не зависит от $t$. Кривые Гурвица $\bar{H}_{1}$ и $\bar{H}_{2}$ называются $H$-деформационно эквивалентными, если существует эквисингулярная $H$-деформация, слоями которой являются кривые $\bar{H}_{1}$ и $\bar{H}_{2}$.

Очевидно, что если точка $p_{t} \in \operatorname{Crit} \bar{H}_{t}$, не являющаяся отрицательным ноудом, принадлежит траектории критических точек эквисингулярной деформации кривой Гурвица $\bar{H}$, то локально эта деформация является эквисингулярной деформацией комплексно-аналитической особенности $\left(\bar{H}_{t} \cup R_{\mathrm{pr}\left(p_{t}\right)}, p_{t}\right)$.

Очевидно также, что разложение на множители брэйд-монодромии кривых Гурвица остается постоянным при эквисингулярной $H$-деформации, т. е. тип разложения на множители брэйд-монодромии кривых Гурвица является инвариантом эквисингулярных $H$-деформаций.

С помощью эквисингулярной деформации мы можем преобразовать кривую Гурвица $\bar{H}$ в кривую Гурвица $\bar{H}_{1}$ такую, что в каждом слое проекции pr лежит не более одной критической точки кривой $\bar{H}_{1}$. Такая деформация меняет разложение на множители брэйд-монодромии кривых Гурвица, и эта замена может быть выражена в терминах гомоморфизма регенерации разложений на множители в группе кос (см. п. 2.4).

5.2. $H$-изотопия кривых Гурвица. Обычно кривые Гурвица рассматриваются с точностью до $H$-изотопии. Две кривые Гурвица $\bar{H}_{1}, \bar{H}_{2} \subset F_{N}$ (возможно, с отрицательными ноудами) называются $H$-изотопными, если существует непрерывная изотопия $\phi_{t}: F_{N} \rightarrow F_{N}, t \in[0,1]$, такая, что

1) $\phi_{0}=\mathrm{Id}$;

2) для каждого $t \in[0,1]$ образ $\phi_{t}\left(\bar{H}_{1}\right)$ является кривой Гурвица и для любого слоя $R_{p}=\operatorname{pr}^{-1}(p)$ точки, принадлежащие $\phi_{t}\left(\operatorname{Crit} \bar{H}_{1} \cap R_{p}\right)$, также лежат в одном слое проекции pr и число этих точек $\#\left(\phi_{t}\left(\operatorname{Crit} \bar{H}_{1} \cap R_{p}\right)\right)$ не зависит от $t$;

3) $\phi_{1}\left(\bar{H}_{1}\right)=\bar{H}_{2}$;

4) $\phi_{t}\left(E_{N}\right)=E_{N}$ для всех $t \in[0,1]$.

Очевидно, имеет место следующее предложение.

ПреДЛОЖЕНИЕ 5.1. Две Н-деформационно эквивалентные кривые Гурвица $\bar{H}_{1}, \bar{H}_{2} \subset F_{N}$ (возможно, с отрицательными ноудами) являются H-изотопными кривыми.

Легко видеть, что при $H$-изотопии кривой Гурвица сохраняется неизменным ее брэйд-монодромный тип разложения на множители.

5.3. Примеры $H$-изотопий. Пусть $h_{t}-$ некоторая гладкая изотопия проективной прямой $\mathbb{C P}^{1}$ такая, что для некоторого заранее фиксированного множества точек $p_{1}, \ldots, p_{n} \in \mathbb{C P}^{1}$ существуют окрестности этих точек $V_{1}, \ldots, V_{n}$ в $\mathbb{C P}^{1}$ такие, что диффеоморфизмы $h_{t}: V_{i} \rightarrow h_{t}\left(V_{i}\right)$ являются комплексно-аналитическими отображениями для всех $t \in[0,1]$ и всех $i=1, \ldots, n$. Так как отображение $\mathrm{pr}: F_{N} \rightarrow \mathbb{C P}^{1}$ является локально тривиальным $C^{\infty}$-расслоением, 
то очевидно, что такая изотопия может быть поднята до гладкой изотопии $\tilde{h}_{t}$ поверхности $F_{N}$ такой, что диффеоморфизмы $\tilde{h}_{t}: \operatorname{pr}^{-1}\left(V_{i}\right) \rightarrow \operatorname{pr}^{-1}\left(h_{t}\left(V_{i}\right)\right)$ являются комплексно-аналитическими отображениями для всех $t \in[0,1]$ и всех $i=1, \ldots, n$. Легко видеть, что если $\left\{p_{1}, \ldots, p_{n}\right\}=\operatorname{pr}(\operatorname{Crit} \bar{H})$ для некоторой кривой Гурвица $\bar{H} \subset F_{N}$, то изотопия $\tilde{h}_{t}$ является $H$-изотопией кривой $\bar{H}$. Такие $H$-изотопии будем называть горизонтальными.

Пусть $h_{t}$ - описанная выше гладкая изотопия проективной прямой $\mathbb{C P}^{1}$. Предположим, что существует такой диск $D_{2}(r) \subset \mathbb{C}^{1} \subset \mathbb{C P}^{1}$ радиуса $r$, что $h_{t}=\mathrm{Id}$ вне диска $D_{2}(r)$ для всех $t \in[0,1]$, и пусть $\left\{p_{1}, \ldots, p_{n}\right\} \subset D_{2}(r)$. Выберем некоторую гладкую монотонную функцию $\lambda:[0, \varepsilon] \rightarrow[0,1], \varepsilon>0$, такую, что $\lambda(\varepsilon)=1$ и $\lambda\left(\varepsilon_{1}\right)=0$ для некоторого $\varepsilon_{1}, 0<\varepsilon_{1}<\varepsilon$, и определим изотопию $\tilde{h}_{t}$ произведения $D_{1}(\varepsilon) \times \mathbb{C P}^{1}$, где $D_{1}(\varepsilon)=\{|u| \leqslant \varepsilon\}-$ диск в $\mathbb{C}^{1}$, положив $\tilde{h}_{t}(u, v)=\left(u, h_{\lambda(|u|) t}(v)\right)$. Очевидно, что если мы отождествим $D_{1}(\varepsilon) \times \mathbb{C P}^{1}$ с прообразом $\operatorname{pr}^{-1}\left(D_{1}(\varepsilon)\right) \subset F_{N}$ некоторого диска $D_{1}(\varepsilon)$, лежащего в $\mathbb{C P}^{1}$, то изотопия $\tilde{h}_{t}$ может быть продолжена до изотопии всей поверхности $F_{N}$, тождественной на дополнении к $\mathrm{pr}^{-1}\left(D_{1}(\varepsilon)\right) \subset F_{N}$. Кроме того, если $\bar{H}-$ кривая Гурвица в $F_{N}$ такая, что $\operatorname{pr}(\operatorname{Crit} \bar{H}) \cap D_{1}(\varepsilon)$ состоит из одной точки - центра диска $D_{1}(\varepsilon)$, то эта изотопия поверхности $F_{N}$ является $H$-изотопией кривой $\bar{H}$. Такие $H$-изотопии будем называть вертикальными.

Частным случаем вертикальной изотопии является следующая изотопия. Пусть $D=I_{1} \times I_{2} \times \bar{D}_{2}(\varepsilon)$, где $I_{1}=[-2,2]=\{-2 \leqslant x \leqslant 2\}$ и $I_{2}=[-2,2]=$ $\{-2 \leqslant y \leqslant 2\}$ - отрезки прямой и $\bar{D}_{2}(\varepsilon)=\{|v| \leqslant \varepsilon\}-$ диск в $\mathbb{C}^{1}$. Обозначим через $h_{1}$ диффеоморфизм пространства $D$, заданный формулой

$$
h_{1}(x, y, v)=\left(x, y,|v| e^{i(\arg (v)+\mu(|v|) \lambda(x) \lambda(y) \varphi)}\right),
$$

где $\mu:[0, \varepsilon] \rightarrow \mathbb{R}$ - гладкая монотонная функция такая, что $\mu(t)=1$ при $t \leqslant \varepsilon / 2$ и $\mu(\varepsilon)=0$, a $\lambda:[-2,2] \rightarrow \mathbb{R}$ - гладкая функция, монотонная на отрезках $[-2,-1]$ и $[1,2], \lambda(-2)=\lambda(2)=0$ и $\lambda(t)=1$ при $-1 \leqslant t \leqslant 1$, и $\varphi-$ некоторый угол. Легко видеть, что $h_{1}$ является тождественным на границе $\partial D$ и, очевидно, он гомотопен тождественному диффеоморфизму $h_{0}=\mathrm{Id}$ в классе диффеоморфизмов пространства $D$, тождественных на границе $\partial D$. Гомотопия $h_{t}, t \in[0,1]$, связывающая $h_{1}$ с $h_{0}=\mathrm{Id}$, задается формулой

$$
h_{1}(x, y, v)=\left(x, y,|v| e^{i(\arg (v)+\nu(t) \mu(|v|) \lambda(x) \lambda(y) \varphi)}\right),
$$

где $\nu:[0,1] \rightarrow \mathbb{R}$ - гладкая монотонная функция такая, что $\nu(0)=0$ и $\nu(1)=1$. Теперь, если пространство $D$ отождествлено с некоторой замкнутой областью $U \subset F_{N}$ так, что проекция pr при этом отождествлении совпадает с проекцией $D \rightarrow I_{1} \times I_{2}$ на первые два сомножителя, то изотопия $h_{t}$ пространства $D \simeq U$ может быть продолжена на все $F_{N}$ как тождественная на дополнении к $U$ в $F_{N}$. Такую изотопию мы будем называть скручиванием-раскручиванием на угол $\varphi$. Очевидно, что при каждом $t$ диффеоморфизм $h_{t}$ является комплексно-аналитическим автоморфизмом пространства $(-1,1) \times(-1,1) \times D_{2}(\varepsilon / 2)$, которое мы будем называть центром скручивания-раскручивания.

Другим примером вертикальной $H$-изотопии является перемасштабирование $h_{t}$, заданное в картах $\mathbb{C}_{i}^{2}$, покрывающих $F_{N} \backslash E_{N}$, формулой $\left(u_{i}, v_{i}\right) \mapsto$ $\left(u_{i}, \lambda(t) v_{i}\right)$, где $\lambda:[0,1] \rightarrow \mathbb{R} \backslash\{0\}-$ некоторая гладкая функция такая, что $\lambda(0)=1$. 
Применяя предложение 1.1, получаем следующее

ПредлОЖениЕ 5.2. Каждая кривая Гурвица $\bar{H} \subset F_{N} H$-изотопна некоторой псевдоголоморфной кривой Гурвица, если $\bar{H}$ не имеет отрицательньх ноудов, и - симплектической кривой Гурвица, если $\bar{H}$ имеет отрицательные ноудъь.

В обозначениях $\S 1$, очевидно, существует гладкая вертикальная $H$-изотопия $h_{t}$, тождественная вне некоторой малой окрестности $U$ слоя $R_{\infty}$, такая, что функция $F_{2}\left(u_{2}, v_{2}, 1\right)$, задающая кривую $\bar{H}_{1}=h_{1}(\bar{H})$ в $\mathbb{C}_{2}^{2}$, совпадает с функцией $v_{2}^{m}-1$ во всех точках $\left(u_{2}, v_{2}\right)$ с $\left|u_{2}\right|<\varepsilon$ для некоторого $\varepsilon>0$. Поэтому далее мы будем предполагать, что кривая $\bar{H}$ задается уравнением $v_{2}^{m}-1=0$ в некоторой окрестности слоя $R_{\infty}$.

Пусть $u_{1, j}$ - критическое значение кривой Гурвица $\bar{H}$, заданной в $\mathbb{C}_{1}^{2}$ уравнением $F_{1}\left(u_{1}, v_{1}\right)=0$, т. е. число различных корней уравнения

$$
F_{1}\left(u_{1, j}, v_{1}\right)=0
$$

строго меньше $m$, и пусть $v_{1, j_{0}}$ - корень уравнения (19) кратности один. Очевидно, существует гладкая вертикальная $H$-изотопия $h_{t}$, тождественная вне маленькой окрестности $U=\left\{\left|u_{1}-u_{1, j}\right|<\varepsilon\right\}$, такая, что функция $F_{1}\left(u_{1}, v_{1}, 1\right)$, определяющая $\bar{H}_{1}=h_{1}(\bar{H})$ в $\mathbb{C}_{1}^{2}$, такова, что $v_{1}=v_{1, j_{0}}$ является корнем уравнения $F_{1}\left(u_{1}, v_{1}, 1\right)=0$ для всех $u_{1}$, удовлетворяющих неравенству $\left|u_{1}-u_{1, j}\right|<\varepsilon_{1}$ при некотором положительном $\varepsilon_{1}<\varepsilon$. Поэтому далее мы можем (и будем) предполагать, что если $\left(u_{1, j}, v_{1, j}\right)$ является критической точкой кривой $\bar{H}$ и не является отрицательным ноудом, то существует такое $\varepsilon>0$, что функция $F_{1}\left(u_{1}, v_{1}\right)$, определяющая кривую $\bar{H}$, является аналитической в точках $\left(u_{1}, v_{1}\right)$ при $\left|u_{1}-u_{1, j}\right|<\varepsilon$.

\section{4. Представление кривых Гурвица $\mathbb{C P}^{2}$ в виде нулей сечений ли-} нейных расслоений. Рассмотрим линейное расслоение $p: \mathscr{L}(m) \rightarrow \mathbb{C P}^{2}$, acсоциированное с пучком $\mathscr{O}_{\mathbb{C P}^{2}}(m)$. Напомним его определение. Проективная плоскость $\mathbb{C P}^{2}$ с однородными координатами $\left(z_{0}: z_{1}: z_{2}\right)$ покрывается тремя картами $\mathbb{C}_{i}^{2}, i=1,2,3$, изоморфными $\mathbb{C}^{2}$, с координатами $\left(u_{i}, v_{i}\right)$, $u_{1}=z_{1} / z_{0}, \quad v_{1}=z_{2} / z_{0}, \quad u_{2}=z_{0} / z_{1}, \quad v_{2}=z_{2} / z_{1}, \quad u_{3}=z_{0} / z_{2}, \quad v_{3}=z_{1} / z_{2}$. Расслоение $\mathscr{L}(m)$ также покрыто тремя картами $W_{i}=\mathbb{C}_{i}^{2} \times \mathbb{C}_{i}^{1}$ с третьей координатой $w_{i}, w_{1}=w_{2} / u_{2}^{m}, w_{1}=w_{3} / u_{3}^{m}, w_{2}=w_{3} / v_{3}^{m}$, и ограничение $p_{\mid W_{i}}$ совпадает с проекцией на первый множитель.

Следующая лемма полезна при построении накрытий проективной плоскости, разветвленных вдоль кривых Гурвица.

Лемма 5.1 [15]. Для любой кривой Гурвица степени $m$ в $\mathbb{C P}^{2}$ относительно линейной проекции с центром в точке $p_{\infty}=(0: 0: 1)$ существует H-изотопная ей кривая Гурвица $\bar{H}$ такая, что функции $w_{i}=F_{i}\left(u_{i}, v_{i}\right), i=$ 1,2 , определяющие кривую $\bar{H}$, задают гладкое сечение s расслоения $\mathscr{L}(m)$ над $\mathbb{C P}^{2} \backslash\left\{p_{\infty}\right\}$, которое может быть продолжено до гладкого сечения $\bar{s}_{m}$ расслоения $\mathscr{L}(m)$ над всем $\mathbb{C P}^{2}$ и для которого существует вещественное число $\varepsilon$, $0<\varepsilon \ll 1$, такое, что: 
i) $\bar{H} \subset \mathbb{C P}^{2} \backslash B_{\varepsilon}$, где $B_{\varepsilon}=\left\{u_{3} \bar{u}_{3}+v_{3} \bar{v}_{3} \leqslant \varepsilon^{2}\right\}-$ шар в $\mathbb{C}_{3}^{2}$ с иентром в $p_{\infty}$;

ii) сечение $\bar{s}_{m}$ является комплексно-аналитическим в некоторой окрестности прямой $L_{\infty}=\left\{z_{0}=0\right\}$ и в некоторой окрестности всех критических точек кривой $\bar{H}$, не являющихся отрицательными ноудами.

5.5. Проблема $H$-изотопности кривых Гурвица. Как уже упоминалось выше, если две кривые Гурвица $H_{1}, H_{2} \subset F_{N}$ являются $H$-изотопными, то эти кривые имеют один и тот же тип разложения на множители брэйд-монодромии. $\mathrm{K}$ настоящему моменту обратное утверждение доказано для кривых Гурвица, имеющих особые точки так называемого нерасщепляемого типа, о которых пойдет речь ниже, а сейчас объясним кратко проблему, возникающую при попытке доказать обратное утверждение.

Пусть две кривые Гурвица $H_{1}, H_{2} \subset F_{N}$ степени $m$ имеют один и тот же тип разложения на множители брэйд-монодромии. Тогда (в обозначениях из п. 4.3) найдется диск $D_{1}(r)$, в котором лежат все критические значения $u_{1}, \ldots, u_{n}$ и

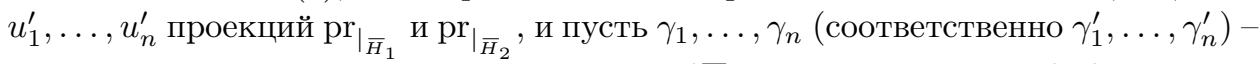
хороший геометрический базис группы $\pi_{1}\left(\bar{D}_{1}(r) \backslash\left\{u_{1}, \ldots, u_{n}\right\}, p_{0}\right)$ (соответственно группы $\left.\pi_{1}\left(\bar{D}_{1}(r) \backslash\left\{u_{1}^{\prime}, \ldots, u_{n}^{\prime}\right\}, p_{0}^{\prime}\right)\right)$, петля $\gamma_{i}=l_{i} \circ \partial D_{1, i}\left(r_{1}\right) \circ l_{i}^{-1}$ (соответственно $\left.\gamma_{i}^{\prime}=l_{i}^{\prime} \circ \partial D_{1, i}^{\prime}\left(r_{1}\right) \circ l_{i}^{\prime-1}\right)$ состоит из пути $l_{i}$ с началом в точке $p_{0}$ и концом в некоторой точке $p_{1, i}$, лежащей на окружности $\partial D_{1, i}\left(r_{1}\right)$ маленького радиуса $r_{1}$ с центром в точке $u_{i}$, обхода вдоль окружности $\partial D_{1, i}\left(r_{1}\right)$ и возврата в точку $p_{0}$ вдоль пути $l_{i}$. Выбрав стандартные порождающие в группах $\mathrm{Br}_{m}\left[D_{2}, K\left(p_{0}\right)\right]=\mathrm{Br}_{m}$ и $\mathrm{Br}_{m}\left[D_{2}, K^{\prime}\left(p_{0}\right)\right]=\mathrm{Br}_{m}$, получим разложения на множители брэйд-монодромий $\operatorname{bmf}\left(H_{1}\right)=b_{1} \cdot \ldots \cdot b_{n}$ и $\operatorname{bmf}\left(H_{2}\right)=b_{1}^{\prime} \cdot \ldots \cdot b_{n}^{\prime}$. Так как $\operatorname{bmt}\left(H_{1}\right)=\operatorname{bmt}\left(H_{2}\right)$, то найдется такой элемент $g \in \operatorname{Br}_{m}$, что $\operatorname{bmf}\left(H_{1}\right)=$ $\lambda(g) \operatorname{bmf}\left(H_{2}\right)$. Очевидно, что найдется такая горизонтальная $H$-изотопия кривой $\bar{H}_{2}$, в результате которой получим такую кривую (обозначим ее снова через $\bar{H}_{2}$ ), что критические значения $u_{i}^{\prime}$ проекции этой кривой совпадают с точками $u_{i}$ для $i=1, \ldots, n, p_{0}^{\prime}=p_{0}$ и относительно базиса $\gamma_{1}, \ldots, \gamma_{n}$ разложение на множители $\operatorname{bmf}\left(H_{2}\right)=b_{1}^{\prime} \cdot \ldots \cdot b_{n}^{\prime}$ таково, что $b_{i}^{\prime}=g^{-1} b_{i} g$ для всех $i=$ $1, \ldots, n$. Далее, применив некоторую вертикальную $H$-изотопию над окрестностями точек $u_{1}, \ldots, u_{n}$ и $p_{0}$, мы можем найти кривую, $H$-изотопную кривой $\bar{H}_{2}$ (обозначим ее снова через $\bar{H}_{2}$ ), которая совпадает с кривой $\bar{H}_{1}$ над окрестностями этих точек, и ее разложение брэйд-монодромии $\operatorname{bmf}\left(H_{2}\right)=b_{1} \cdot \ldots \cdot b_{n}$ относительно базиса $\gamma_{1}, \ldots, \gamma_{n}$. Для каждого пути $l_{i}$, соединяющего точку $p_{0}$ с точкой $p_{i}$, тривиализуем расслоение $\mathrm{pr}: \mathrm{pr}^{-1}\left(l_{i}\right) \simeq l_{i} \times \mathbb{C P}^{1} \rightarrow l_{i}$ так, чтобы $\operatorname{pr}_{2}\left(K\left[p_{0}\right]\right)=\operatorname{pr}_{2}\left(K\left[p_{i}\right]\right)$, где $\mathrm{pr}_{2}$ - это проекция на $\mathbb{C P}^{1}$ тривиального расслоения $l_{i} \times \mathbb{C P}^{1}$. Тогда каждый элемент $b_{i}$ в разложении $\operatorname{bmf}\left(H_{1}\right)=b_{1} \cdot \ldots \cdot b_{n}$ равен $b_{i}=g_{i, 1} b_{i, 0} g_{i, 1}^{-1}$, где $g_{i, 1}=\bar{H}_{1} \cap \mathrm{pr}^{-1}\left(l_{i}\right)$ - это геометрическая коса над путем $l_{i}$ и $b_{i, 0}$ - это коса над $\partial D_{1, i}\left(r_{1}\right)$, и элемент $b_{i}$ в разложении $\operatorname{bmf}\left(H_{2}\right)=b_{1} \cdot \ldots \cdot b_{n}$ равен $b_{i}=g_{i, 2} b_{i, 0} g_{i, 2}^{-1}$, где $g_{i, 2}=\bar{H}_{2} \cap \mathrm{pr}^{-1}\left(l_{i}\right)$. Так как $b_{i}=g_{i, 1} b_{i, 0} g_{i, 1}^{-1}=g_{i, 2} b_{i, 0} g_{i, 2}^{-1}$, то элемент $g_{i, 1}^{-1} g_{i, 2}$ коммутирует с элементом $b_{i, 0}$ и проблема состоит в доказательстве существования вертикальной $H$-изотопии ростка кривой Гурвица над ее критическим значением, которая "раскручивала" бы геометрическую косу $g$, коммутирующую с брэйд-монодромией $b$ этого ростка. 
5.6. Критерий $H$-изотопности каспидальных кривых Гурвица. Следующая лемма описывает централизатор $C\left(a_{1}^{k}\right)$ степеней стандартного порождающего элемента $a_{1} \in \mathrm{Br}_{m}$.

Лемма 5.2 [39]. Для любого $k \in \mathbb{Z} \backslash\{0\}$ иентрализатор $C\left(a_{1}^{k}\right) \subset \mathrm{Br}_{m}$ элемента $a_{1}^{k}$ порождается полуповоротами $a_{1}, a_{3}, a_{4}, \ldots, a_{m-1}$ и полными поворотами $\Delta_{3,0}^{2}, \ldots, \Delta_{m, 0}^{2}$, где $a_{1}, \ldots, a_{m-1}$ - стандартные порождающие груп$n$ øı $\mathrm{Br}_{m}$.

Из леммы 5.2 следует, что для критической точки типа $A_{k}$ любой элемент $b \in C\left(a_{1}^{k}\right)$ может быть "раскручен" с помощью композиции нескольких скручиваний-раскручиваний на углы, кратные $\pi$, причем каждое из этих скручиваний-раскручиваний либо является изотопией, тождественной в окрестности критической точки, либо имеет своим центром критическую точку. Аналогичное утверждение имеет место для слоев над точкой, стандартная форма брэйд-монодромии которых имеет вид $a_{1}^{k_{1}} a_{3}^{k_{2}} \ldots a_{2 l-1}^{k_{l}}$. Отсюда вытекает следующий результат.

ТеОрема 5.1 [39], [20]. Каспидальные кривые Гурвица $\bar{H}_{1}, \bar{H}_{2} \subset F_{N}$ гладко Н-изотопнь тогда и только тогда, когда $\bar{H}_{1}$ и $\bar{H}_{2}$ имеют один и тот же тип разложения брэйд-монодромии на множители.

СледСТВиЕ 5.1. Любая каспидальная кривая Гурвица $\bar{H}_{1} \subset F_{N}$ без отрицательных ноудов гладко $Н$-изотопна почти алгебрачческой кривой.

Вопрос о том, определяет ли брэйд-монодромный тип разложения на множители алгебраической каспидальной кривой $\bar{H} \subset \mathbb{C P}^{2}$ дифференцируемый тип пары $\left(\mathbb{C P}^{2}, \bar{H}\right)$, был исследован в работе [40]. В этой работе было показано, что существуют диффеоморфные пары $\left(\mathbb{C P}^{2}, \bar{H}_{1}\right)$ и $\left(\mathbb{C P}^{2}, \bar{H}_{2}\right)$, однако кривые $\bar{H}_{1}$ и $\bar{H}_{2}$ имеют не совпадающие типы брэйд-монодромных разложений на множители (см. пример в 8.1). Эти примеры показывают, что брэйд-монодромный тип разложения на множители кривой является инвариантом, различающим кривые с точностью до $H$-изотопии.

5.7. $H$-изотопность кривых Гурвица с особыми точками нерасщепляемого типа. Доказывая $H$-изотопность кривых Гурвица с критическими точками нерасщепляемого типа, имеющих одинаковый тип разложения на множители брэйд-монодромии, удается избежать описания централизатора $C(b)$ в группе кос монодромии $b$ ростка критической точки кривой Гурвица. Росток критической точки кривой Гурвица, стандартная форма монодромии которого равна $b \in \mathrm{Br}_{k}$, называется нерасщепляемым, если при стандартном действии группы кос $\mathrm{Br}_{k}$ на свободной группе $\mathbb{F}_{k}=\left\langle x_{1}, \ldots, x_{k}\right\rangle$ множество неподвижных элементов $\mathbb{F}_{k}^{b}=\left\langle w \in \mathbb{F}_{k} \mid(w) b=w\right\rangle$ совпадает с циклической подгруппой $\langle l\rangle \subset \mathbb{F}_{k}$, порожденной элементом $l=x_{1} \ldots x_{k}$.

Имеет место следующая теорема.

Теорема 5.2 [20]. Две кривые Гурвица с особенностями нерасщепляемого типа, имеющие в каждом слое не более одной критической точки, являются Н-изотопными тогда и только тогда, когда они имеют одинаковые типь брэйд-монодромии. 
Стратегия доказательства этой теоремы совпадает в основном с описанной в п. 5.5 стратегией. Единственное отличие состоит в том, что на последнем шаге доказательства, чтобы избежать описания централизаторов монодромий критических точек нерасщепляемого типа, используется следующая лемма.

Лемма 5.3. Пусть $b \in \mathrm{Br}_{k}$ - нерасщепляемый элемент $u\left\{x_{1}, \ldots, x_{m}\right\}$ геометрический базис группь $\pi_{1}\left(D_{2} \backslash\left\{v_{1}, \ldots, v_{m}\right\}\right)$. Интерпретируем $b$ как элемент группы $\operatorname{Br}_{k, 0}=\operatorname{Br}\left[D_{2},\left\{v_{1}, \ldots, v_{k}\right\}\right] \subset B\left[D_{2},\left\{v_{1}, \ldots, v_{m}\right\}\right]=\operatorname{Br}_{m}$ u pacсмотрим индуиированное элементом $b$ действие на $\pi_{1}=\pi_{1}\left(D_{2} \backslash\left\{v_{1}, \ldots, v_{m}\right\}\right)$. Тогда подгруппа $F(b)$ неподвижных элементов в $\pi_{1}$ относительно действия $b$ порождается элементами $l=x_{1} \ldots x_{k} u x_{k+1}, \ldots, x_{m}$.

ДоКАЗАТЕЛЬСтво. Очевидно, что $l$ и $x_{k+i}$ с $i \geqslant 1$ принадлежат группе $F(b)$. Пусть $g \in F(b)$. Запишем элемент $g$ как приведенное слово, состоящее из букв $x_{1}, \ldots, x_{m}$ и обратных к ним, $g=s_{1} s_{2} \ldots s_{n}$, где каждое $s_{i}$ - приведенное слово, непустое, если $i \neq 1$, состоящее из букв $x_{1}, \ldots, x_{k}$ и обратных к ним, если $i-$ нечетное число, и из $x_{k+1}, \ldots, x_{m}$ и обратных к ним, если $i$ четно. Так как такое представление единственно и $g \in F(b)$, то каждый элемент $s_{i}$ принадлежит $F(b)$, и доказательство леммы вытекает из определения нерасщепляемых элементов.

С каждым ростком $W$-подготовленной аналитической особенности $(D, C)$, $D=D_{1} \times D_{2}$, линк которой $L \subset \partial D_{1} \times D_{2}$ реализует монодромию $b \in \mathrm{Br}_{k}$ этой особенности, можно связать стандартную коническую модель топологической особенности типа $b$. Она задается ростком $H=C(L)$, где

$$
C(L)=\{(r u, r v) \mid 0 \leqslant r \leqslant 1,(u, v) \in L\} .
$$

Хорошо известно, что если $(D, C)$ - росток $W$-подготовленной аналитической особенности, то росток $(D, C, \mathrm{pr})$ гомеоморфен конической особенности типа $b=\operatorname{pr}^{-1}\left(\partial D_{1} \cap C\right)$ и, более того, имеет место следующее утверждение.

Лемма 5.4. Для любой особенности $\left(D=D_{1} \times D_{2}, H, \mathrm{pr}\right)$ существует изотопия, оставляющая неподвижным каждый слой $\mathrm{pr}$, тождественная над дD и преобразующая особенность в ее стандартную коническую модель $C(L)$, $L=H \cap\left(\partial D_{1} \times D_{2}\right)$.

Применяя леммы 5.3 и 5.4, на последнем шаге доказательства теоремы 5.2 доказываем существование вертикальной $H$-изотопии, которая "раскручивает" геометрическую косу $g$, принадлежащую централизатору нерасщепляемой монодромии $b$.

Ответ на вопрос: "Являются ли нерасщепляемъми стандартные формы монодромий всех аналитических особенностей?" к настоящему моменту не известен. Однако множество типов аналитических особенностей, стандартные формы монодромий которых нерасщепляемы, как показывает следующая лемма, является достаточно большим.

ЛЕмма 5.5. Особенность алгебраической кривой, заданная уравнением $v^{k}=u^{n}$, является нерасщепляемой для всех $n \geqslant 1 u k \geqslant 1$. 
ДокАзАТЕЛЬСтво. Согласно лемме 4.1 стандартная форма такой особенности равна $b=\left(a_{1} \ldots a_{k-1}\right)^{n} \in \mathrm{Br}_{k}$, где $a_{1}, \ldots, a_{k-1}-$ множество стандартных порождающих группы кос $\mathrm{Br}_{k}$. Равенство $\Delta_{k}^{2}=\left(a_{1} \ldots a_{k-1}\right)^{k}$ влечет $b^{k}=\Delta_{k}^{2 n}$. $\mathrm{C}$ другой стороны, действие элемента $\Delta_{k}^{2}$ совпадает с действием сопряжением на элемент $\partial D_{2} \in \pi_{1}$. Теперь осталось заметить, что централизатор любого элемента $g \neq 1$ в свободной группе совпадает с максимальной циклической подгруппой, содержащей элемент $g$.

5.8. Изотопность псевдоголоморфных кривых в $\mathbb{C P}^{2}$. Как известно, $\mathbb{C P}^{2}$ обладает единственной с точностью до симплектоморфизмов и умножения на постоянный множитель симплектической структурой. Эта структура задается формой Фубини-Штуди $\omega_{0}$.

Пусть $C \subset \mathbb{C P}^{2}-J$-голоморфная кривая, т. е. $C$ является образом $C=$ $f(\Sigma)$ приведенной $J$-голоморфной кривой $f: \Sigma \rightarrow \mathbb{C P}^{2}$, где $J-$ некоторая почти комплексная структура, определенная в некоторой окрестности поверхности $C$, являющаяся ручной относительно заданной симплектической структуры $\omega$ на $\mathbb{C P}^{2}$, и, кроме того, комплексная структура на $\Sigma$ индуцирована структурой $J$. Говорят, что две особые симплектические поверхности $C_{0}=$ $f_{0}(\Sigma), C_{1}=f_{1}(\Sigma)$ являются слабо симплектически (соответственно гладко) изотопными, если $\left(f_{0}, J_{0}^{\text {loc }}, \omega_{0}\right),\left(f_{1}, J_{1}^{\text {loc }}, \omega_{1}\right)$ могут быть включены в непрерывное (соответственно гладкое) семейство ручных почти комплексных и симплектических структур $J_{t}^{\mathrm{loc}}, \omega_{t}$ и отображений $J_{t}^{\text {loc }}$-голоморфных кривых $f_{t}$ : $\Sigma \times[0,1] \rightarrow \mathbb{C P}^{2}$ такое, что отображения $\phi_{t}: C_{0} \rightarrow C_{t}$, заданные по правилу $f_{0}(s) \mapsto f_{t}(s), s \in \Sigma$, являются хорошо определенными гомеоморфизмами для всех $t \in[0,1]$. Аналогичные определения применимы к симплектическим поверхностям на любом многообразии. В случае, когда симплектическая структура не изменяется, говорят о симплектической изотопии. В частности, две особые симплектические поверхности симплектически изотопны, если существует симплектическая диффеотопия, преобразующая одну поверхность в другую.

ЗАмЕчАНИЕ 5.1. В случае произвольных симплектических многообразий естественно ожидать, что классы симплектически изотопных поверхностей и классы слабо симплектически изотопных поверхностей не совпадают в общем случае. В противоположность этому, эти понятия совпадают в случае симплектических структур на $\mathbb{C P}^{2}$. Действительно, в этом случае любая слабо симплектическая изотопия может быть (не изменяя $J_{t}$ ) перемасштабирована, $\omega_{t} \mapsto \omega_{t}^{\prime}=\lambda_{t} \omega_{t}, \quad \lambda_{t} \in \mathbb{R}_{+}$, в изотопию с $\omega_{t}^{\prime}$, не меняющими свой класс когомологий; после этого остается применить теорему Мозера и получить диффеотопию, делающую $\omega_{t}^{\prime}$ постоянной формой; та же самая диффеотопия затем применяется к $f_{t}$ и $J_{t}^{\text {loc }}$. Поэтому, когда нам нужно доказать, что некоторые симплектические поверхности в $\mathbb{C P}^{2}$ симплектически изотопны, нам достаточно проверить, что они слабо симплектически изотопны.

ЗАмечАниЕ 5.2. Пространство почти комплексных структур на конечномерном вещественном векторном пространстве, являющихся ручными относительно заданной симплектической формы, является стягиваемым, см. [37]. Отсюда следует, что любая $\omega$-ручная почти комплексная структура $J^{\text {loc }}$, определенная на открытом подмножестве $U$ симплектического многообразия $(V, \omega)$, 
может быть продолжена с более маленькой окрестности $U_{0} \subset U$ до $\omega$-ручной почти комплексной структуры $J$ на всем $V$. Более того, по той же причине, если $U=U_{t}, J^{\text {loc }}=J_{t}^{\text {loc }}$ и $\omega=\omega_{t}$ гладко зависят от одного или нескольких параметров $t$, это продолжение с $U_{0}=U_{0, t}$ на $\mathbb{C P}^{2}$ может быть выбрано гладко зависящим от $t$. Следовательно, мы получим те же самые понятия, если в данных выше определениях заменим определенные локально $J$-структуры на ручные почти комплексные структуры, определенные на всем $\mathbb{C P}^{2}$. Выбор определений, который мы сделали, мотивирован упрощением некоторых доказательств. Отметим также, что приведенные выше свойства продолжения остаются верными, если дополнительно ограничиться случаем почти комплексных структур, для которых данная симплектическая поверхность является $J$-голоморфной. В частности, в определении слабой симплектической изотопии поверхностей достаточно требовать, чтобы $J_{t}^{\text {loc }}$ были определены только вблизи особых точек поверхностей $C_{t}$.

Как отмечалось выше, особенности псевдоголоморфных кривых на почти комплексных четырехмерных многообразиях эквивалентны с точностью до $C^{1}$-замены координат особенностям настоящих комплексных кривых, лежащих на комплексных поверхностях. В случае, когда псевдоголоморфная кривая $C$ задана вместе с симплектической структурой $\omega$ и $\omega$-ручной почти комплексной структурой $J^{\text {loc }}$ в некоторой окрестности $U$ кривой $C$ в $\mathbb{C P}^{2}$, локальная структура $J^{\text {lос }}$ может быть продолжена с более маленькой окрестности $U_{0} \subset U$ до $\omega$-ручной почти комплексной структуры $J$ на всем $\mathbb{C P}^{2}$, а после этого можно рассмотреть общий (относительно кривой $C$ ) пучок $L$, составленный из $J$-прямых (см. [37] и [41]), и определить брэйд-монодромный тип $\operatorname{bmt}(C, \omega, J, L)$ (см. п. 4.5).

Отметим, что любой элемент $s \in \mathscr{P}$ такой, что $\alpha(s)=\Delta_{m}^{2}$ (и только такие элементы), может быть реализован как разложение на множители брэйдмонодромии некоторой псевдоголоморфной кривой относительно некоторого (не обязательно общего) пучка. Такая реализация может быть получена из почти алгебраической кривой с таким же разложением $s$ брэйд-монодромии (см. теорему 4.2) после перемасштабирования стандартного пучка прямых, относительно которого эта почти алгебраическая кривая задана.

ПРЕДЛОЖЕНИЕ 5.3. Тип брэйд-монодромии $\operatorname{bmt}(C, \omega, J, L)$ относителъно общего пучка $L$ зависит только от класса симплектически изотопных кривых, которому принадлежит $C$. (В частности, он не зависит от продолжения $J$ на все $\mathbb{C P}^{2}$ почти комплексной структуры $J^{\mathrm{loc}}$, определенной локально.)

ДоказАТЕЛЬСтво. Пусть $C_{0}, C_{1}$ - слабо симплектически изотопные симплектические поверхности, снабженные $\omega_{i}$-ручными $(i=0,1) J$-структурами $J_{0}^{\text {loc }}, J_{1}^{\text {loc }}$, и пусть $J_{0}, J_{1}$ - продолжения этих структур и $L_{0}, L_{1}$ - общие пучки такие же, как и в определениях особых симплектических поверхностей и соответствующих им разложений брэйд-монодромий на множители соответственно. Выберем слабую симплектическую изотопию $\left(C_{t}, \omega_{t}, J_{t}^{\mathrm{loc}}\right)$ и продолжим $J_{t}^{\text {loc }}$ до изотопии $J_{t}$ на все $\mathbb{C P}^{2}$. Для каждого $t$ пространство $\left(\mathbb{C P}_{t}^{2}\right)^{*}$, составленное из $J_{t}$-прямых, диффеоморфно $\mathbb{C P}^{2}$ и снабжено канонической двойственной $E$-структурой (см. [41]). Касательные $J_{t}$-прямые к $C_{t}$ составляют 
двойственную $E$-кривую $C_{t}^{*}$. Она имеет конечное число особых точек (которые соответствуют двойным касательным или касательным с более сильным касанием), и двойственная к $C_{t}^{*}$ поверхность совпадает с $C_{t}$. Поэтому пучок является общим, если его центр не принадлежит ни $C_{t}$, ни конечному числу $J_{t}$-прямых, двойственных особым точкам кривой $C_{t}^{*}$. Следовательно, найдется путь $L_{t}$, состоящий из общих пучков и соединяющий $L_{0}$ с $L_{1}$. Это завершает доказательство, так как ясно, что разложения на множители брэйд-монодромий кривых $C_{t}$ относительно пучков $L_{t}$ не зависят от $t$.

Следующая теорема, доказанная в [20], является частично обратным утверждением к предложению 5.3.

ТеОРема 5.3. Симплектические относительно симплектической структуры Фубини-Штуди обыкновенные каспидальные поверхности являются симплектически $C^{1}$-гладко изотопными в $\mathbb{C P}^{2}$ тогда и только тогда, когда они имеют один и тот же тип разложения брэйд-монодромии относительно общих пучков.

\section{9. Сравнение множеств алгебраических и псевдоголоморфных} кривых в $\mathbb{C P}^{2}$. Каждая алгебраическая кривая в $\mathbb{C P}^{2}$ может быть рассмотрена как псевдоголоморфная кривая. Поэтому возникает вопрос о том, на сколько больше имеется псевдоголоморфных кривых, рассматриваемых с точностью до симплектической изотопии, по сравнению с алгебраическими кривыми.

На настоящий момент примером псевдоголоморфной кривой $\bar{H}$ наименьшей степени $\operatorname{deg} \bar{H}=7$, не изотопной алгебраической кривой, является следующая кривая. Для ее построения рассмотрим алгебраическую кривую $C=C_{1} \cup L_{1} \cup$ $L_{2} \cup L_{3}$, где $C_{1}-$ кривая степени 4 , имеющая три обыкновенных каспа $s_{1}, s_{2}$ и $s_{3}$, а $L_{i}$ - это прямые, проходящие через точки $s_{i}$ и имеющие локальный индекс пересечения $\left(C_{1}, L_{i}\right)_{s_{i}}=3$.

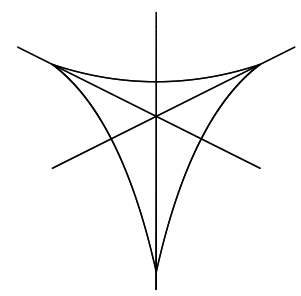

Рис. 3

Как известно, все прямые $L_{i}$ проходят через некоторую точку $s_{0}$ и такая конфигурация кривых является жесткой, т. е. каждая такая конфигурация получается из другой такой конфигурации с помощью проективного преобразования плоскости. Мы можем слегка пошевелить кривую $C$ так, что после шевеления получится симплектическая поверхность $\bar{H}=\bar{H}_{1} \cup \bar{L}_{1} \cup \bar{L}_{2} \cup \bar{L}_{3}$, где $\bar{H}_{1}$ - симплектическая поверхность степени 4 , имеющая три обыкновенных каспа $s_{1}, s_{2}$ и $s_{3}$, а $\bar{L}_{i}$ - это прямые, проходящие через точки $s_{i}$ и имеющие 
локальный индекс пересечения $\left(\bar{H}_{1}, \bar{L}_{i}\right)_{s_{i}}=3$, но прямые $\bar{L}_{i}$ уже не имеют общей точки. Очевидно, что в силу жесткости кривой $C$ кривая $\bar{H}$ не изотопна алгебраической кривой.

Пример псевдоголоморфной неприводимой кривой $\widetilde{H}$ наименьшей (известной к настоящему моменту) степени $\operatorname{deg} \widetilde{H}=8$, не изотопной алгебраической кривой, получается из предыдущего примера: если мы произведем квадратичное преобразование плоскости $\tau: \mathbb{C P}^{2} \rightarrow \mathbb{C P}^{2}$ (которое раздувает точки пересечения прямых $\bar{L}_{i}$ и $\bar{L}_{j}, i \neq j$, и после этого стягивает в точки собственные прообразы прямых $\bar{L}_{i}$ ), то получим симплектическую поверхность $\widetilde{H}-$ собственный прообраз поверхности $\bar{H}_{1}, \operatorname{deg} \widetilde{H}=8$. Легко видеть, что поверхность $\widetilde{H}$ имеет три особые точки $\bar{s}_{i}$, локальные уравнения которых имеют вид $\left(u^{3}-v^{5}\right)(u+2 v)=0$. Так как кратность каждой особой точки равна 4 и $\operatorname{deg} \widetilde{H}=8$, то точки $\bar{s}_{i}$ не могут лежать на одной прямой. Поэтому псевдоголоморфная кривая $\widetilde{H}$ не может быть изотопна алгебраической кривой, так как иначе мы бы сделали квадратичное преобразование плоскости $\tau: \mathbb{C P}^{2}-\rightarrow \mathbb{C P}^{2}$ с центром в точках $\bar{s}_{i}$ и получили бы алгебраическую кривую $\bar{C}, \operatorname{deg} \bar{C}=4$, имеющую три каспа, касательные прямые $L_{i}$ к которой в этих трех каспах не имели бы общей точки.

Б. Мойшезон (см. [13; теорема 1]; см. также [42]) доказал существование бесконечной последовательности попарно различных типов обыкновенных каспидальных (т. е. каждый множитель сопряжен элементу $a_{1}^{k}, 1 \leqslant k \leqslant 3$ ) разложений на множители элемента $\Delta_{54}^{2}$. Отсюда и из теоремы 4.2 следует

ПреДЛОЖеНИЕ 5.4. Существует бесконечная последовательность $\bar{H}_{i} \subset F_{1}$, $i \in \mathbb{N}$, общих обыкновенных каспидалъных почти алгебраических (u, тем самым, псевдоголоморфных) кривых степени 54 с ровно 378 каспами и 756 ноудами и с попарно различными типами разложений брэйд-монодромий на множители. В частности, все они не $H$-изотопны друг другу, почти все из них не изотопны алгебраическим каспидальным кривым.

Отметим, что в случае обыкновенных каспидальных алгебраических кривых Гурвица степени $m$ с фиксированными числами обыкновенных каспов, ноудов и простейших точек касания со слоями проекции $\mathrm{pr}$ в $F_{N}$ существует лишь конечное число различных типов $H$-изотопных кривых. Например, для алгебраических кривых в $\mathbb{C P}^{2}$ степени 6 с шестью обыкновенными каспами имеется ровно два типа разложений на множители брэйд-монодромии (так называемая пара Зариского), которые различаются с помощью групп, ассоциированных с разложениями на множители.

$\mathrm{K}$ настоящему моменту ответ на следующий вопрос является неизвестным. Существует ли тройка натуральных чисел $(m, c, n)$, для которой существует псевдоголоморфная кривая $\bar{H}, \operatorname{deg} \bar{H}=m$, имеющая с обыкновенных каспов u $n$ ноудов в качестве своих особых точек, но не существует алгебраической кривой $C$ с теми же инвариантами $(m, c, n)$ ?

Для каспидальной кривой $C$ четной степени $\operatorname{deg} C=2 d$ с $c$ обыкновенными каспами и $n$ ноудами имеет место следующее неравенство (см. [43]):

$$
9 n+16 c \leqslant 2 d(10 d-6) .
$$


Очень интересен следующий вопрос: существует ли псевдоголоморфная каспидальная кривая $\bar{H}$ четной степени, для которой не выполнено неравенство (20)?

В случае нодальных кривых Гурвица степени $m$ в $F_{N}$ (т. е. кривых Гурвица, все критические точки которых - либо положительные ноуды, либо простейшие точки касания со слоями проекции pr) ответ на вопрос о числе не изотопных типов до сих пор является открытым. Однако из теоремы Севери (см. [44]) для нодальных кривых в проективной плоскости и ее обобщения на нодальные кривые, лежащие в $F_{N}$ (см. [45]), вытекает следующий результат.

Теорема 5.4. Две нодальные алгебрачческие кривые Гурвица в $F_{N}$ cmeneни $\mathrm{m}$ Н-изотопны тогда и только тогда, когда они принадлежат одной и той же неприводимой компоненте пространства нодальных кривых с фиксированным числом ноудов. В частности, две неприводимые нодальные алгебраические кривые Гурвица степени $m$ H-изотопны тогда и толъко тогда, когда они имеют одинаковое число особых точек.

Кроме того, имеет место следующая теорема.

Теорема 5.5 [46]. Любые две нодалъные кривые Гурвица в $F_{N}$, являющиеся объединением $m$ сечений проекции $\mathrm{pr}, \mathrm{H}$-изотопны.

Эти две теоремы, а также утверждения о том, что неособые кривые Гурвица в $F_{1}$ (т. е. в $\mathbb{C P}^{2}$ ) степени $m \leqslant 17$ (см. [47]) и неприводимые нодальные кривые геометрического рода нуль $H$-изотопны алгебраическим кривым [48], позволяют надеяться, что следующая гипотеза имеет положительное решение.

ГипотезА 5.1. Каждая нодальная кривая Гурвища в $F_{N} H$-изотопна алгебрачческой кривой.

Теорема 5.5, предложение 4.2 и утверждение 4.2 влекут

СЛЕДСТВИЕ 5.2. Пусть элемент $s \in \mathscr{A}_{1} \subset S_{\mathrm{Br}_{m}}$ таков, что $\alpha(s)=\Delta_{m}^{2 N}$. Тогда $s=\left(\tilde{\delta}^{2}\right)^{N}$.

Заметим, что для доказательства гипотезы 5.1 достаточно показать, что любое нодальное (т. е. в $S_{A_{0} \cup A_{1}}$ ) разложение на множители элемента $\Delta^{2 N}$ может быть получено как частичная регенерация (см. п. 2.4) элемента $\left(\tilde{\delta}^{2}\right)^{N} \in \mathscr{A}_{1}$.

5.10. Полугруппа $\operatorname{Hur}_{m}$ над группой кос $\mathrm{Br}_{m}$. Kaк и в случае отмеченных разветвленных накрытий диска (см. введение), на множестве кривых Гурвица над диском с некоторой дополнительной структурой, рассматриваемых с точностью до сильной $H$-изотопии (см. ниже), можно также ввести структуру полугруппы над группой кос.

А именно, рассмотрим множество кривых Гурвица $H \subset \bar{D} \times \mathbb{C}^{1}$ степени $m$ над диском $\bar{D}=\left\{u \in \mathbb{C}^{2}|| u \mid \leqslant 1\right\}$ таких, что Crit $H \subset \bar{D} \backslash \partial \bar{D}$. Зафиксируем точку $p_{0}=\{u=-i\} \in \partial \bar{D}$. Для каждой кривой Гурвица $H$ слой над точкой $p_{0}$ - это пара $\left(\mathbb{C}^{1}, \mathrm{pr}^{-1}\left(p_{0}\right) \cap H\right)$. Выберем для каждой кривой Гурвица $H$ диффеоморфизм $\varphi:\left(\mathbb{C}^{1},\left\{q_{1}, \ldots, q_{m}\right\}\right) \rightarrow\left(\operatorname{pr}^{-1}\left(p_{0}\right), \operatorname{pr}^{-1}\left(p_{0}\right) \cap H\right)$, где $\left\{q_{1}, \ldots, q_{m}\right\} \subset \mathbb{C}^{1}-$ некоторое, также фиксированное, множество точек. Пару $(H, \varphi)$ будем называть оснащенной кривой Гурвица над диском. Скажем, 
что оснащенные кривые Гурвица $\left(H_{0}, \varphi_{0}\right)$ и $\left(H_{1}, \varphi_{1}\right)$ сильно $H$-изотопны, если существует $H$-изотопия $h_{t}$ такая, что $h_{t}\left(\mathrm{pr}^{-1}\left(p_{0}\right)\right)=\left(\mathrm{pr}^{-1}\left(p_{0}\right)\right)$ для всех $t$ и $h_{1} \circ \varphi_{0}=\varphi_{1}$.

Очевидно, что для каждой оснащенной кривой Гурвица $(H, \varphi)$ существует сильно $H$-изотопная ей кривая $(\widetilde{H}, \widetilde{\varphi})$ такая, что $\widetilde{\varphi}=\mathrm{Id}$, и, кроме того, можно считать, что $\widetilde{H}=H^{\prime}$ или $\widetilde{H}=H^{\prime \prime}$, где $H^{\prime}$ (соответственно $\left.H^{\prime \prime}\right)$ - это такая кривая, что найдется окрестность $U \subset \bar{D}$ правой полуокружности $\{u \in \partial \bar{D} \mid$ $\operatorname{Re} u \geqslant 0\}$ (соответственно левой полуокружности $\{u \in \partial \bar{D} \mid \operatorname{Re} u \leqslant 0\}$ ), над которой $H^{\prime}$ (соответственно $H^{\prime \prime}$ ) является объединением $m$ постоянных сечений $v=q_{i}$.

Введем на множестве $\mathrm{Hur}_{m}$ классов эквивалентности оснащенных кривых Гурвица относительно сильной $H$-изотопии структуру полугруппы: для двух классов $\overline{\left(H_{1}, \varphi_{1}\right)}$ и $\overline{\left(H_{2}, \varphi_{2}\right)}$ произведение $\overline{(H, \varphi)}=\overline{\left(H_{1}, \varphi_{1}\right)} \cdot \overline{\left(H_{2}, \varphi_{2}\right)}$ определяется следующим образом. Выберем таких представителей $\left(H_{1}^{\prime}, \mathrm{Id}\right)$ и $\left(H_{2}^{\prime \prime}, \mathrm{Id}\right)$ классов $\overline{\left(H_{1}, \varphi_{1}\right)}$ и $\overline{\left(H_{2}, \varphi_{2}\right)}$, как было определено выше, и пусть $\psi_{j}, j=1,2,-$ гладкие вложения диска $\bar{D}$ в себя такие, что $\psi_{1}$ (соответственно $\psi_{2}$ ) является голоморфным отображением в окрестности критических значений проекции $\mathrm{pr}$ для кривой $H_{1}^{\prime}$ (соответственно для $\left.H_{2}^{\prime \prime}\right)$ и, кроме того,

(i) $\psi_{j}\left(p_{0}\right)=p_{0}$ при $j=1,2$;

(ii) образ $\psi_{1}(\bar{D})=\bar{D}_{+}=\{u \in \bar{D} \mid \operatorname{Re} u \geqslant 0\}$ - это правый полукруг и $\psi_{1}(\{u \in \partial \bar{D} \mid \operatorname{Re} u \leqslant 0\})=\{u \in \bar{D} \mid \operatorname{Re} u=0\}$;

(iii) $\psi_{2}(\bar{D})=\bar{D}_{-}=\{u \in \bar{D} \mid \operatorname{Re} u \leqslant 0\}-$ это левый полукруг и $\psi_{2}(\{u \in \partial \bar{D} \mid \operatorname{Re} u \geqslant 0\})=\{u \in \bar{D} \mid \operatorname{Re} u=0\}$.

Склеив расслоения $\psi_{1} \circ \operatorname{pr}:\left(\bar{D} \times \mathbb{C}^{1}, H_{1}^{\prime}\right) \rightarrow \bar{D}_{+}$и $\psi_{2} \circ \operatorname{pr}:\left(\bar{D} \times \mathbb{C}^{1}, H_{2}^{\prime \prime}\right) \rightarrow \bar{D}_{-}$ вдоль диаметра $\{u \in D \mid \operatorname{Re} u=0\}$, получим оснащенную кривую Гурвица $(H, I d)$, класс которой равен произведению $\overline{\left(H_{1}, \varphi_{1}\right)} \cdot \overline{\left(H_{2}, \varphi_{2}\right)}$.

Очевидно, что полугруппа $\operatorname{Hur}_{m}$ порождается классами $\overline{(H, \varphi)}$, представители которых имеют только одну критическую точку.

Выберем $r \gg 0$ такое, что диск $D_{2}=\left\{v \in \mathbb{C}^{1}|| v \mid<r\right\}$ содержит все точки $q_{1}, \ldots, q_{m}$, и для каждого класса $\overline{(H, \varphi)}$, представители которого имеют только одну критическую точку, выберем такого представителя $\left(H^{\prime}, \mathrm{Id}\right)$, что $H^{\prime} \subset$ $\bar{D}_{1} \times D_{2}$, и положим $\operatorname{bmf}(\overline{(H, \varphi)})=\operatorname{bmf}\left(H^{\prime}\right) \in \operatorname{Br}\left[\bar{D}_{2},\left\{q_{1}, \ldots, q_{m}\right\}\right]=\operatorname{Br}_{m}$. Очевидно, что это отображение продолжается до гомоморфизма bmf: $\operatorname{Hur}_{m} \rightarrow S_{\mathrm{Br}_{m}}$ и, кроме того, определяет на $\mathrm{Hur}_{m}$ структуру полугруппы над группой кос $\mathrm{Br}_{m}$.

Полугруппа $\operatorname{Hur}_{m}$ содержит подполугруппу $\operatorname{Hur}_{A, m}$, элементы которой - это классы, представители которых имеют только критические точки типа $A$. Из теоремы 5.1 следует, что bmf: $\operatorname{Hur}_{A, m} \rightarrow \mathscr{A}$ является изоморфизмом полугрупп над группой $\mathrm{Br}_{m}$.

\section{§ 6. Регулярная гомотопия кривых Гурвица}

6.1. Рождение и уничтожение пары ноудов. Кроме $H$-изотопии приходится рассматривать (см. §9) и такие деформации, при которых с кривой Гурвица происходит "стандартное" преобразование, состоящее либо из возникновения (рождения) новой, либо из уничтожения некоторой старой пары ноудов, имеющих противоположные знаки. 
Рождением пары ноудов вдоль простого пути $\gamma$ называется преобразование кривой Гурвица $\bar{H}=\bar{H}_{t^{*}-\tau}$, заданное с помощью гомотопии $\bar{H}_{t}, t \in\left[t^{*}-\tau\right.$, $\left.t^{*}+\tau\right]$ и $0<\tau \ll 1$, удовлетворяющей следующим условиям:

1) кривые $\bar{H}_{t}$ изотопны друг другу вне некоторой окрестности $U$ пути $\gamma$;

$2)$ в $U$ существуют такие вещественные координаты $(x, y, u, v)$, что проекция pr: $F_{N} \rightarrow \mathbb{C P}^{1}$ в этих координатах совпадает с отображением pr: $(x, y, u, v) \rightarrow(x, y)$, а путь $\gamma$ совпадает с путем $\{u \in[-\tau, \tau], x=$ $v=y=0\}$

3) для каждого $t \in\left[t^{*}-\tau, t^{*}+\tau\right]$ кривая $\bar{H}_{t} \cap U$ состоит из двух дисков, которые являются графиками сечений

$$
s_{t}^{ \pm}:(x, y) \mapsto(u, v)=\left( \pm\left(x^{2}-\left(t-t^{*}\right)\right), \pm y\right) .
$$

Диск, заданный формулой $D:=\left\{v=y=0, x \in[-\sqrt{\tau}, \sqrt{\tau}], x^{2}-\tau \leqslant u \leqslant\right.$ $\left.\tau-x^{2}\right\}$, называется диском Уитни, вдоль которого происходит рождение пары ноудов.
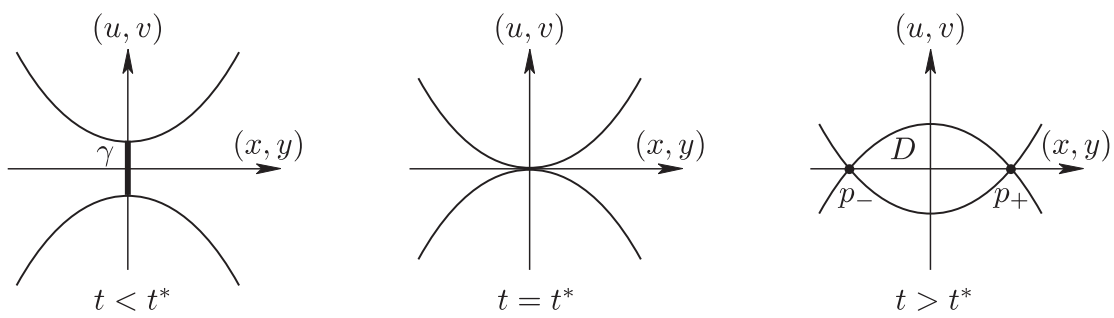

Рис. 4

Обратное преобразование называется уничтожением пары ноудов вдоль диска Уитни D.

Путь $\gamma$ лежит в слое $\{x=y=0\}$ и соединяет две точки, лежащие на кривой $\bar{H}=\bar{H}_{t^{*}-\tau}$; "рожденные" ноуды $p_{-}$и $p_{+}$имеют противоположные ориентации: один из них - положительный, а другой - отрицательный. Обращение течения времени $t$ на противоположное заменяет операцию рождения ноудов на уничтожение пары ноудов.

Две кривые Гурвица называются регулярно гомотопными, если одна из них может быть получена из другой с помощью композиции конечного числа $H$-изотопий, рождений и уничтожений пар ноудов.

Обоснованием данного определения является следующее утверждение, доказательство которого является легким упражнением.

Лемма 6.1. Пусть $\phi_{t}: \Sigma \rightarrow F_{N}$ - гладкая гомотопия отображений замкнутой ориентированной вещественной поверхности $\Sigma$ в $F_{N}$, обладающая следующими свойствами:

1) для каждого $t$ композииия $\mathrm{pr} \circ \phi_{t}: \Sigma \rightarrow \mathbb{C P}^{1}$ является разветвленным накрытием;

2) отображение $\phi_{t}$ является $H$-изотопией в некоторой окрестности всех критических точек отображения $\mathrm{pr} \circ \phi_{t}$; 
3) отображение $\phi_{t}$ является общим относительно условий 1) и 2). Тогда $\phi_{t}$ является регулярной гомотопией.

Следующая лемма описывает условия, достаточные для рождения пары ноудов.

Лемма 6.2. Пусть $\bar{H}$ - кривая Гурвица в $F_{N}, R-$-лой проекиии pr, не содержащий критических точек кривой $\bar{H}, \gamma \subset R$ - простой путь в $R \backslash \bar{H}$, концы которого лежат на $\bar{H}, U$ - произвольная окрестность пути $\gamma$ в $F_{N}$. Тогда существует регулярная гомотопия $\phi_{t}$ кривой $\bar{H}$, в результате которой рождается пара ноудов вдоль пути $\gamma$ и которая является постоянной вне $U$.

ДокАЗАТЕЛЬСтво. Из условий леммы следует, что путь $\gamma$ пересекает кривую $\bar{H}$ трансверсально. Уменьшая, если необходимо, окрестность $U$, можно выбрать такие локальные координаты $(x, y, u, v)$ в $U$, удовлетворяющие условию 2) из определения рождения пары ноудов, что $U \cap \bar{H}$ состоит из двух дисков, являющихся графиками непересекающихся сечений $s_{t^{*}-\tau}^{ \pm}:(x, y) \mapsto(u, v)=$ $\left( \pm\left(x^{2}+\tau\right), \pm y\right)$. Лемма 6.2 доказана.

Легко видеть, что если кривая Гурвица $\bar{H}_{2}$ получена из кривой Гурвица $\bar{H}_{1}$ в результате рождения пары ноудов, то разложения на множители брэйд-монодромий кривых Гурвица $\bar{H}_{1}$ и $\bar{H}_{2}$ связаны следующим соотношением:

$$
\operatorname{bmf}\left(\bar{H}_{2}\right)=\operatorname{bmf}\left(\bar{H}_{1}\right) \cdot g \cdot\left(g^{-1}\right),
$$

где $g$ - некоторый элемент из $A_{1}$.

Следующая лемма показывает, что верно и обратное утверждение.

Лемма 6.3 [46]. Пусть $\bar{H}-$ кривая Гурвица в $F_{N} u p_{+}, p_{-} \in \operatorname{Crit}(\bar{H})-\partial в е$ нодальные особые точки такие, что слои расслоения $F_{N}$, проходящие через точки $p_{ \pm}$, не проходят через другие точки из $\operatorname{Crit}(\bar{H})$. Предположим, что найдется простой гладкий путь $\gamma \subset \mathbb{C P}^{1}$, удовлетворяющий следующим условиям:

1) концы пути $\gamma$ совпадают с проекциями $q_{ \pm}:=\operatorname{pr}\left(p_{ \pm}\right)$нодальных точек $p_{ \pm}$

2) открытый путь $\gamma$ не проходит через точки, лежащие в $\operatorname{pr}(\operatorname{Crit}(\bar{H}))$;

3) пусть $q_{0} \in \mathbb{C P}^{1} \backslash \operatorname{Crit}(\bar{H})$ - базисная точка и $R_{0}-$ слой расслоения $F_{N}$ над точкой $q_{0}, \gamma_{-} \subset \mathbb{C P}^{1} \backslash \operatorname{Crit}(\bar{H})$ - гладко вложенный путь от точки $q_{0}$ до точки $q_{-}, \gamma_{+} \subset \mathbb{C P}^{1} \backslash \operatorname{Crit}(\bar{H})$ - путь от точки $q_{0}$ до $q_{+}$, полученный как композиция путей $\gamma_{-} u \gamma, u$ пусть $\mu_{ \pm}-$брэйд-монодромии кривой $\bar{H}$ в группе кос $\operatorname{Br}_{m}\left(R_{0}\right)$ в точках $p_{ \pm}$вдоль путей $\gamma_{ \pm}$связаны соотношением $\mu_{+} \cdot \mu_{-}=1 \in \operatorname{Br}_{m}\left(R_{0}\right)$.

Тогда эта пара нодалъных точек $\left(p_{+}, p_{-}\right)$может быть уничтожена с помощъю регулярной гомотопии $\phi_{t}$, постоянной вне произвольной заранее выбранной окрестности $U \subset F_{N}$ множества $\operatorname{pr}^{-1}(\gamma)$.

Легко видеть, что уничтожение пары ноудов вдоль пути $\gamma$ - если оно возможно - зависит только от класса изотопных путей с фиксированными концами, которому принадлежит $\gamma$. Заметим также, что условие, наложенное на монодромию в лемме 6.3 , является необходимым и достаточным условием для 
потенциальной возможности уничтожить пару ноудов. Скажем, что два элемента $s_{1}, s_{2} \in S_{\mathrm{Br}_{m}}$ слабо эквивалентны, если $c\left(s_{1}\right)=c\left(s_{2}\right)$, где $c: S_{\mathrm{Br}_{m}} \rightarrow$ $\bar{S}_{\mathrm{Br}_{m}, A_{1} \cup A_{-3}}$ - гомоморфизм сокращения, определенный в п. 2.2. В качестве следствия получаем следующее утверждение.

ПреДЛОЖенИЕ 6.1. Две кривые Гурвица $\bar{H}_{0}, \bar{H}_{1} \subset F_{N}$ степени $m$ являются регулярно гомотопными тогда и только тогда, когда $\operatorname{bmf}\left(\bar{H}_{1}\right)$ u $\operatorname{bmf}\left(\bar{H}_{2}\right)$ слабо эквивалентны.

\section{2. Критерий регулярной гомотопности каспидальных кривых} Гурвица. Пусть $\bar{H} \subset F_{N}-$ каспидальная кривая Гурвица, $\operatorname{bmf}(\bar{H})=s_{-3}$. $s_{0} \cdot s_{1} \cdot \ldots \cdot s_{k}$, где $s_{i} \in \mathscr{A}_{i}=S_{A_{i}}$. Пусть $l_{i}$ - длина элемента $s_{i}$ (возможно, некоторые $\left.l_{i}=0\right)$. Упорядоченные наборы чисел $t(\bar{H})=\left(l_{-3}, l_{1}, l_{2}, \ldots, l_{k}\right)$ и $\bar{t}(\bar{H})=\left(l_{1}-l_{-3}, l_{2}, \ldots, l_{k}\right)$ называются соответственно типом и приведенным типом множества особых точек кривой $\bar{H}$.

ТеОрема 6.1 [46]. Любые две неприводимые каспидалъные кривые Гурвица $\bar{H}_{1}, \bar{H}_{2} \subset F_{N}$ степени $m$ являются регулярно гомотопными тогда и только тогда, когда они имеют одинаковые приведенные типь множества особых точек.

Более того, если $\bar{H}_{0}$ и $\bar{H}_{1}$ являются симплектическими поверхностями относительно некоторой симплектической формы $\omega$, совместимой с каноническим расслоением $F_{N}$ на проективные прямые $\mathbb{C P}^{1}$, то регулярная гомотопия, связывающая эти поверхности, может быть выбрана $\omega$-симплектической.

Приведем набросок одного из доказательств этой теоремы.

Очевидно, что если каспидальные кривые Гурвица $\bar{H}_{1}, \bar{H}_{2} \subset F_{N}$ являются регулярно гомотопными, то они имеют одинаковые приведенные типы множества особых точек.

Пусть $\bar{H}_{1}$ и $\bar{H}_{2}$ имеют одинаковые приведенные типы множества особых точек $\bar{t}=\left(\bar{l}_{1}, l_{2}, \ldots, l_{k}\right)$. Рассмотрим накрытия $\operatorname{pr} \circ f_{i}: \Sigma_{i} \rightarrow \mathbb{C P}^{1}, i=1,2$, где $f_{i}: \Sigma_{i} \rightarrow F_{N}$ - отображения, участвующие в определении кривых Гурвица. Эти накрытия разветвлены в прообразах $f_{i}^{-1}\left(p_{i, j}\right)$ точек $p_{i, j} \in \operatorname{Crit} \bar{H}_{i}$, монодромия которых $b_{i, j} \in A_{n}$ с четными $n$, причем порядок ветвления в каждой из этих точек равен двум. Из формулы Гурвица следует, что поверхности $\Sigma_{1}$ и $\Sigma_{2}$ имеют одинаковый род $g$, равный $\sum_{n \geqslant 0} l_{2 n}-m+1$, так как $l_{0}$ определяется однозначно по приведенному типу множества особых точек: $l_{0}=N m(m-1)-$ $\sum_{n \geqslant 2}(n+1) l_{n}-2 \bar{l}_{1}$.

Рассмотрим естественный гомоморфизм $\gamma_{m}: \mathrm{Br}_{m} \rightarrow \mathscr{S}_{m}$ в симметрическую группу $\mathscr{S}_{m}$. Элементы $z_{k, l}^{2}, 1 \leqslant k<l \leqslant m$, порождают ker $\gamma_{m}=P_{m}$ - группу крашенных кос, где $z_{k, l}=\left(a_{l-1} \ldots a_{k+1}\right) a_{k}\left(a_{l-1} \ldots a_{k+1}\right)^{-1}$ для $k<l$.

Согласно теореме Гурвица имеем $\gamma_{m *}\left(\operatorname{bmf}\left(\bar{H}_{1}\right)\right)=\gamma_{m *}\left(\operatorname{bmf}\left(\bar{H}_{2}\right)\right)=h_{m, g}$, где $h_{m, g}$ - элемент Гурвица рода $g$ (см. п. 2.1). Заметим, что образ $\gamma_{m}(b) \in \mathscr{S}_{m}$ является либо транспозицией, если $b \in A_{n}$ с четным $n$, либо он равен $\mathbf{1} \in \mathscr{S}_{m}$, если $b \in A_{n}$ с нечетным $n$. Если $\operatorname{bmf}\left(\bar{H}_{i}\right)=b_{i, 1} \cdot \ldots \cdot b_{i, l}$, то по предположению теоремы для $i=1,2$ образы $\gamma_{m}\left(b_{i, j}\right)$ элементов $b_{i, j} \in \bigcup_{n=0}^{\infty} A_{2 n}$ порождают группу $\mathscr{S}_{m}$. 
Так как множители, входящие в разложения на множители элемента $h_{m, g}$, порождают всю группу $\mathscr{S}_{m}$, то, неоднократной перестановкой множителей в $\operatorname{bmf}\left(\bar{H}_{i}\right)$ мы можем добиться того, что $\operatorname{bmf}\left(\bar{H}_{i}\right)=s_{i \text {,even }} \cdot s_{i, \text { odd }}$, где

$$
s_{i, \text { odd }}=s_{i,-3} \cdot s_{i, 1} \cdot \ldots \cdot s_{i, \min (2[k / 2]+1, k)}, \quad s_{i, \text { even }}=s_{i, 0} \cdot s_{i, 2} \cdot \ldots \cdot s_{i, 2[k / 2]}
$$

и где $s_{i, j}=b_{i, j, 1} \cdot \ldots \cdot b_{i, j, d_{j}} \in \mathscr{A}_{j}$, причем для нечетных $j$ каждое из $b_{i, j, l}$ равно $g_{i, j, l} z_{1,2}^{j+1} g_{i, j, l}^{-1}$, где все $g_{i, j, l} \in P_{m}$.

Лемма 6.4 [46]. Пусть элемент $b \in A_{0}$ имеет тот же самый образ в $\mathscr{S}_{m}$, что и $z_{k_{0}, l_{0}}$, m.е. $\gamma_{m}(b)=\gamma_{m}\left(z_{k_{0}, l_{0}}\right)=\left(k_{0}, l_{0}\right)$. Тогда существует элемент $p \in P_{m}$ такой, что $b=p z_{k_{0}, l_{0}} p^{-1}$. Более того, элемент $p$ можно выбрать таким, что он может быть записан как положительное слово в алфавиme $\left\{z_{i, j}^{2}\right\}$.

Согласно предложению 6.1 элементы $\bar{s}_{M_{1}, i}=\operatorname{bmf}\left(\bar{H}_{i}\right) \cdot\left(\tilde{\delta}^{2} \cdot \tilde{\delta}^{-2}\right)^{M}$ являются разложениями на множители брэйд-монодромии кривых, регулярно гомотопных кривым $\bar{H}_{1}$ и $\bar{H}_{2}$, где

$$
\tilde{\delta}_{m}^{2}=\prod_{l=m}^{2} \prod_{k=1}^{l-1} z_{k, l}^{2} \in S_{A_{1}}, \quad \tilde{\delta}_{m}^{-2}=\prod_{l=2}^{m} \prod_{k=l-1}^{1} z_{k, l}^{-2} \in S_{A_{-3}} .
$$

Для любых $i$ и $j$ элемент $\tilde{\delta}_{m}^{2}$ можно записать в виде $\tilde{\delta}_{m}^{2}=z_{i, j}^{2} \cdot \delta_{i, j}$ с некоторым $\delta_{i, j} \in \mathscr{A}_{1}$ (так как согласно предложению 4.2 элемент $\tilde{\delta}_{m}^{2}$ инвариантен при сопряжении), и, кроме того, согласно предложению 2.1 имеем $s \cdot \tilde{\delta}^{2}=\tilde{\delta}^{2} \cdot s$ и $s \cdot \tilde{\delta}^{-2}=\tilde{\delta}^{-2} \cdot s$ для всех $s \in S_{\mathrm{Br}_{m}}$. Поэтому, применив лемму $6.4 \mathrm{k} \mathrm{мно-}$ жителям элементов $\bar{s}_{M, i}$ с достаточно большим $M \in \mathbb{N}$, с помощью неоднократных перестановок множителей, входящих в $\tilde{\delta}^{2}$, можно показать, что $\bar{s}_{M, i}=s \cdot\left(s_{i,+} \cdot s_{i,-}\right) \cdot s^{\prime} \cdot\left(\tilde{\delta}^{-2}\right)^{M}$, где $s^{\prime} \in \mathscr{A}_{1}, \alpha(s)=\Delta^{2 N}$ и $c\left(s_{i,+} \cdot s_{i,-}\right)=\mathbf{1}$. А так как $\alpha\left(\bar{s}_{M, i}\right)=\Delta_{m}^{2 N}$, то $\alpha\left(s^{\prime}\right)=\Delta_{m}^{2 M}$ и из теоремы 5.5 следует, что $s^{\prime}=\left(\tilde{\delta}^{2}\right)^{M}$. Отсюда вытекает регулярная гомотопность кривых $\bar{H}_{1}$ и $\bar{H}_{2}$.

Следующее утверждение является следствием из теорем 6.1 и 5.3.

СЛЕДСТВИЕ 6.1. Пусть $C_{0}$ и $C_{1}$ - две обикновенные каспидалъные неприводимые симплектические поверхности в $\left(\mathbb{C P}^{2}, \omega_{0}\right), \operatorname{deg} C_{0}=\operatorname{deg} C_{1}$, псевдоголоморфные относительно $\omega_{0}$-ручных почти комплексных структур $J_{0}$ и $J_{1}$ соответственно. Если $C_{0}$ и $C_{1}$ имеют совпадающие числа ноудов и каспов, то они могут быть продебормированы одна в другую с помощью $C^{1}-$ гладкой симплектической гомотопии в $\mathbb{C P}^{2}$.

\section{§ 7. Фундаментальные группы дополнений к кривым Гурвица}

7.1. Теоремы Зариского-ван Кампена. Одним из инвариантов вложения кривой Гурвица $\bar{H}$ в поверхность Хирцебруха $F_{N}$ является фундаментальная группа $\pi_{1}\left(F_{N} \backslash \bar{H}\right)$. Зная разложение на множители $\operatorname{bmf}(\bar{H})$ брэйд-монодромии кривой $\bar{H}$, легко выписать копредставление этой группы.

Действительно, пусть $\bar{D}=\bar{D}_{1}\left(r_{1}\right) \times \bar{D}_{2}\left(r_{2}\right)$ - это замкнутый бидиск в $F_{N}$ такой, что ограничение на $\bar{H} \cap D$ проекции $\operatorname{pr}: \bar{D} \rightarrow \bar{D}_{1}\left(r_{1}\right)$ является 
собственным отображением степени $m$, и предположим, что $\operatorname{pr}(\operatorname{Crit} \bar{H}) \cap$ $\partial \bar{D}=\varnothing$. Как и в п. 4.3, обозначим через $u_{1}, \ldots, u_{n} \in D_{1}\left(r_{1}\right)$ критические значения проекции $\mathrm{pr}_{\mid \bar{H}}$, лежащие в $D_{1}=D_{1}\left(r_{1}\right)$, зафиксируем некоторую точку $p_{0} \in \partial \bar{D}_{1}$ и выберем хороший геометрический базис $\gamma_{1}, \ldots, \gamma_{n}$ группы $\pi_{1}\left(\bar{D}_{1} \backslash\right.$ $\left.\left\{u_{1}, \ldots, u_{n}\right\}, p_{0}\right)$. Зафиксировав хороший геометрический базис $x_{1}, \ldots, x_{m}$ группы $\pi_{1}\left(\bar{D}_{2} \backslash K\left(p_{0}\right), q_{0}\right)$, где $K\left(p_{0}\right)=\bar{H} \cap \mathrm{pr}^{-1}\left(p_{0}\right), D_{2}=D_{2}\left(r_{2}\right)$ и $q_{0} \in \partial \bar{D}_{2}$, и выбрав порождающие (полуповороты) $a_{1}, \ldots, a_{m-1}$ группы $\operatorname{Br}_{m}\left[D_{2}, K\left(p_{0}\right)\right]$, стандартно действующие на базисе $x_{1}, \ldots, x_{m}$ свободной группы $\pi_{1}\left(\bar{D}_{2} \backslash\right.$ $\left.K\left(p_{0}\right), q_{0}\right)$, получим разложение на множители $\operatorname{bmf}\left(\bar{H}, D_{1}\right)=b_{1} \cdot \ldots \cdot b_{n}$ брэйд-монодромии кривой $\bar{H}$ над диском $D_{1}$.

Удалив слои $R_{u_{i}}$ над критическими точками $u_{1}, \ldots, u_{n}$, получим локально тривиальное $C^{\infty}$-расслоение

$$
\operatorname{pr}: \bar{D} \backslash\left(\bar{H} \cup\left(\bigcup_{i} R_{u_{i}}\right)\right) \rightarrow \bar{D}_{1} \backslash\left\{u_{1}, \ldots, u_{n}\right\} .
$$

Следовательно, имеет место точная последовательность групп

$1 \rightarrow \pi_{1}\left(\bar{D}_{2} \backslash K\left(p_{0}\right), q_{0}\right) \rightarrow \pi_{1}\left(\bar{D} \backslash\left(\bar{H} \cup\left(\bigcup_{i} R_{u_{i}}\right)\right), q_{0}\right) \rightarrow \pi_{1}\left(\bar{D}_{1} \backslash\left\{u_{1}, \ldots, u_{n}\right\}, p_{0}\right) \rightarrow 1$,

т. е. группа $\pi_{1}\left(\bar{D} \backslash\left(\bar{H} \cup\left(\bigcup_{i} R_{u_{i}}\right)\right), q_{0}\right)$ есть расширение свободной группы $\pi_{1}\left(\bar{D}_{1} \backslash\right.$ $\left.\left\{u_{1}, \ldots, u_{n}\right\}, p_{0}\right)=\left\langle\gamma_{1}, \ldots, \gamma_{n}\right\rangle$ с помощью свободной группы $\left\langle x_{1}, \ldots, x_{m}\right\rangle=$ $\pi_{1}\left(\bar{D}_{2} \backslash K\left(p_{0}\right), q_{0}\right)$. Отождествив диск $\bar{D}_{1}$ с диском $\mathrm{pr}_{2}^{-1}\left(\mathrm{pr}_{2}\left(q_{0}\right)\right) \subset \bar{D}$, где $\mathrm{pr}_{2}-$ это проекция бидиска $\bar{D}$ на второй сомножитель $\bar{D}_{2}$, получаем вложение группы $\pi_{1}\left(\bar{D}_{1} \backslash\left\{u_{1}, \ldots, u_{n}\right\}, p_{0}\right)$ в группу $\pi_{1}\left(\bar{D} \backslash\left(\bar{H} \cup\left(\bigcup_{i} R_{u_{i}}\right)\right), q_{0}\right)$. Легко видеть, что при этом вложении $\gamma_{j} x_{i} \gamma_{j}^{-1}=\left(x_{i}\right) b_{j}$ для всех $i=1, \ldots, m$ и $j=1, \ldots, n$, т. е. группа $\pi_{1}\left(\bar{D} \backslash\left(\bar{H} \cup\left(\bigcup_{i} R_{u_{i}}\right)\right), q_{0}\right)$ имеет следующее копредставление:

$$
\begin{aligned}
\pi_{1}\left(\bar{D} \backslash\left(\bar{H} \cup\left(\bigcup_{i} R_{u_{i}}\right)\right), q_{0}\right)=\left\langle x_{1}, \ldots, x_{m}, \gamma_{1}, \ldots, \gamma_{n}\right| \\
\\
\left.\quad \gamma_{j} x_{i} \gamma_{j}^{-1}=\left(x_{i}\right) b_{j}, i=1, \ldots, m, j=1, \ldots, n\right\rangle .
\end{aligned}
$$

В качестве следствия получаем следующую теорему.

Теорема 7.1. Пусть $\bar{H}$ - кривая Гурвица степени $m$ на поверхности Хирцебруха $F_{N}$, и пусть $\bar{D}=\bar{D}_{1}\left(r_{1}\right) \times \bar{D}_{2}\left(r_{2}\right)$ - это замкнутый бидиск в $F_{N}$ такой, что ограничение на $\bar{H} \cap \bar{D}$ проекции $\mathrm{pr}: \bar{D} \rightarrow \bar{D}_{1}\left(r_{1}\right)$ является собственным отображением степени $m$. Тогда фундаменталъная группа $\pi_{1}(\bar{D} \backslash \bar{H})$ является C-группой и, более того,

$$
\pi_{1}(\bar{D} \backslash \bar{H}) \simeq G(s)=\left\langle x_{1}, \ldots, x_{m} \mid x_{i}=\left(x_{i}\right) b_{j}, i=1, \ldots, m, j=1, \ldots, n\right\rangle,
$$

где $s=\operatorname{bmf}\left(\bar{H}, D_{1}\right)=b_{1} \cdot \ldots \cdot b_{n}-$ разложение на множители брэйд-монодромии кривой $\bar{H}$ над диском $D_{1}, a G(s)$ - группа, ассоциированная с элементом $s \in S_{\mathrm{Br}_{m}}$.

Из теорем 3.4 и 4.1 следует обратное утверждение: любая $C$-группа $G$ может быть реализована как фундаментальная группа $\pi_{1}(\bar{D} \backslash \bar{H})$ для некоторой кривой Гурвица $\bar{H}$. Более того, имеет место следующая теорема. 
Теорема 7.2. Каждая $C$-группа $G$ может быть реализована как фундаментальная группа $\pi_{1}(\bar{D} \backslash C)$ для некоторой неособой алгебрачческой кривой $C \subset F_{N}$

Устремляя $r_{1}$ и $r_{2}$ в теореме 7.1 к бесконечности, получаем следующую теорему.

ТЕОРЕМА ЗАРИСКОГО-ВАН КАМПЕНА. Пусть $\bar{H} \subset F_{N}-$ кривая Гурвица степени $m u \operatorname{bmf}(\bar{H})=s$ - разложение на множители ее брэйд-монодромии. Tогда:

$$
\pi_{1}\left(\mathbb{C}^{2} \backslash \bar{H}\right) \simeq G(s)
$$

где $\mathbb{C}^{2}=F_{N} \backslash\left(E_{N} \cup R_{\infty}\right), u$

$$
\begin{aligned}
& \pi_{1}\left(F_{N} \backslash \bar{H}\right) \simeq G(s) /\left\{\left(x_{1} \ldots x_{m}\right)=\mathbf{1}\right\} \\
& \quad=\left\langle x_{1}, \ldots, x_{m} \mid x_{1} \ldots x_{m}=\mathbf{1}, x_{i}=\left(x_{i}\right) b_{j}, i=1, \ldots, m, j=1, \ldots, n\right\rangle .
\end{aligned}
$$

Техника брэйд-монодромных разложений на множители была применена в восьмидесятые годы прошлого столетия Б. Мойшезоном и М. Тайхер к вычислению фундаментальных групп дополнений к плоским алгебраическим кривым во многих конкретных примерах. В частности, ими были вычислены фундаментальные группы дополнений к кривым ветвления общих проекций поверхностей на проективную плоскость (определение общей проекции см. в §8) в следующих случаях: для проекций неособых поверхностей, лежащих в $\mathbb{C P}^{3}$; для проекций плоскости $\mathbb{C P}^{2}$, вложенной с помощью вложений Веронезе в проективные пространства; для проекций квадрики $\mathbb{C P}^{1} \times \mathbb{C P}^{1}$, вложенной в проективные пространства с помощью линейных систем $\left|a l_{1}+b l_{2}\right|$ [6]-[13]. Отметим, что вычисление этих фундаментальных групп позволило им построить контрпример к известной в свое время гипотезе о том, что алгебраические поверхности общего типа, инварианты которых удовлетворяют неравенству $c_{1}^{2}>2 c_{2}$, не являются односвязными (см. [49]).

Согласно предложению 4.3 и лемме 3.7 фундаментальные группы $\pi_{1}\left(\mathbb{C}^{2} \backslash H\right)$ к аффинным кривым Гурвица $H$ являются гурвицевскими $C$-группами, так как $\alpha(s)=\Delta_{m}^{2 N}$ для $s=\operatorname{bmf}(\bar{H})$, если $\bar{H} \subset F_{N}$. Из теорем 3.5, 4.2 и леммы 4.1 следует обратное утверждение.

Теорема 7.3. Для каждой гурвищевской $C$-группъ $G$ степени $m$ существуют натуральное число $n$ и полуалгебрачческая кривая Гурвица $\bar{H} \subset \mathbb{C P}^{2}$ степени $2^{n} m$ с особыми точками типа $v^{m}=u^{m}$ такие, что $\pi_{1}\left(\mathbb{C}^{2} \backslash H\right)$ $C$-изоморфна группе $G$.

Доказательства приведенных выше теорем дословно переносятся на случай псевдоголоморфных кривых в $\mathbb{C P}^{2}$.

ТЕОРЕМА ЗАРИСКОГО-ВАН КАМПЕНА (ПсевдоГОЛОМОфНЫЙ случай). Пусть $\bar{H} \subset \mathbb{C P}^{2}$ - псевдоголоморфная кривая степени $m$, разложение на множители брэйд-монодромии которой относительно некоторого пучка псевдоголоморфных прямых равно $\operatorname{bmf}(\bar{H})=b_{1} \cdot \ldots \cdot b_{n}$. Тогда

$$
\pi_{1}\left(\mathbb{C P}^{2} \backslash\left(\bar{H} \cup R_{\infty}\right)\right) \simeq G(\operatorname{bmf}(\bar{H})),
$$


где $R_{\infty}$ - псевдоголоморфная прямая, принадлежащая этому пучку и находящаяся в общем положении относительно кривой $\bar{H}, u$

$$
\begin{gathered}
\pi_{1}\left(\mathbb{C P}^{2} \backslash \bar{H}\right) \simeq\left\langle x_{1}, \ldots, x_{m}\right| x_{1} \ldots x_{m}=\mathbf{1}, \\
\left.x_{i}=\left(x_{i}\right) b_{j}, i=1, \ldots, m, j=1, \ldots, n\right\rangle .
\end{gathered}
$$

Применяя перемасштабирование, мы можем считать, что почти алгебраическая кривая $\bar{H}$ является симплектической поверхностью. Поэтому из теоремы 7.3 вытекает следующий результат.

Теорема 7.4. Для каждой гурвицевской $C$-группы $G$ степени $m$ существуют натуральное число $n$, псевдоголоморфная кривая $\bar{H} \subset \mathbb{C P}^{2}$ степени $2^{n} m$ с особыми точками типа $v^{m}=u^{m}$ и псевдоголоморфная прямая $R_{\infty}$ такие, что $\pi_{1}\left(\mathbb{C P}^{2} \backslash\left(\bar{H} \cup R_{\infty}\right)\right) C$-изоморфна группе $G$.

7.2. Проблема Зариского. Пусть $C$ - плоская алгебраическая кривая. В [50] О. Зариский сформулировал следующий вопрос: является ли группа $G=\pi_{1}\left(\mathbb{C P}^{2} \backslash C\right)$ финитно аппроксимируемой группой? (Группа $G$ называется финитно аппроксимируемой, если для любых двух элементов $g_{1}, g_{2} \in G$ найдутся конечная группа $\widetilde{G}$ и эпиморфизм $h: G \rightarrow \widetilde{G}$ такие, что $h\left(g_{1}\right) \neq h\left(g_{2}\right)$.)

Естественно задать аналогичный вопрос в локальном случае, т. е. в случае $G=\pi_{1}(D \backslash C)$, где $D$ - некоторый бидиск в $\mathbb{C}^{2}$, а также в случае, когда $G$ фундаментальная группа дополнения к кривой Гурвица или псевдоголоморфной кривой в $\mathbb{C P}^{2}$. Ответ на данный вопрос в алгебраическом случае до сих пор неизвестен. Однако во всех остальных случаях ответ отрицателен.

Хорошо известно (см., например, [33; теорема 4.10]), что конечно порожденная финитно аппроксимируемая группа является хопфовой (напомним, что группа называется хопфовой, если она не изоморфна никакой своей собственной факторгруппе). Так, например, неприводимые $C$-группы

$$
G_{k}=\left\langle x_{1}, x_{2} \mid\left(x_{1}^{-1} x_{2}\right)^{k} x_{1}\left(x_{1}^{-1} x_{2}\right)^{-k} x_{2}^{-1}=\mathbf{1}\right\rangle,
$$

$k \in \mathbb{N}$, не являются хопфовыми группами при $k \geqslant 2$, и, следовательно, они не являются финитно аппроксимируемыми. (Группа $G_{k}$ изоморфна группе Баумслага-Солитера $\left\langle a, x_{1} \mid x_{1}^{-1} a^{k} x_{1} a^{-(k+1)}=\mathbf{1}\right\rangle$ (см. [51]), если положить $x_{2}=x_{1} a$.) Согласно теореме 7.2 каждая из этих $C$-групп может быть реализована как фундаментальная группа $\pi_{1}(\bar{D} \backslash C)$ для некоторой неособой алгебраической кривой $C \subset F_{N}$.

Используя группы Баумслага-Солитера, можно получить примеры гурвицевских $C$-групп, не являющихся хопфовыми группами (см. [27]). Так, например, группа

$$
\begin{gathered}
\widetilde{G}_{k}=G_{k} \times \mathbb{F}_{1} \simeq\left\langle x_{1}, x_{2}, x_{3}\right|\left(x_{1}^{-1} x_{2}\right)^{k} x_{1}\left(x_{1}^{-1} x_{2}\right)^{-k}=x_{2}, \\
\left.\left[\left(x_{1} x_{2} x_{3}\right), x_{i}\right]=\mathbf{1}, i=1,2,3\right\rangle
\end{gathered}
$$

является гурвицевской $C$-группой степени 3 , составленной из двух неприводимых компонент. Применяя теорему 7.3, получаем следующее утверждение. 
Теорема 7.5 [27]. Существует полуалгебраическая кривая Гурвица $\bar{H} \subset$ $\mathbb{C P}^{2}$, состоящая из двух неприводимых компонент, все особые точки которой являются простейшими тройными точками, т.е. локально они могут быть заданы уравнением $v\left(v^{2}-u^{2}\right)=0, u$ для которой группь $\pi_{1}\left(\mathbb{C P}^{2} \backslash \bar{H}\right)$ $u \pi_{1}\left(\mathbb{C}^{2} \backslash H\right)$ являются нехопфовыми $u$, в частности, эти группы не являются финитно аппроксимируемыми.

Применяя перемасштабирование, мы можем считать, что $\bar{H}$ является симплектической поверхностью. Поэтому имеет место следующая теорема.

Tеорема 7.6 [27]. Существует псевдоголоморфная кривая $\bar{H} \subset \mathbb{C P}^{2}$ с простейшими тройными особыми точками, состоящая из двух неприводимых компонент, для которой группа $\pi_{1}\left(\mathbb{C P}^{2} \backslash \bar{H}\right)$ является нехопфовой.

\section{§ 8. Алгебраические разветвленные накрытия проективной плоскости}

8.1. Монодромия накрытия. По определению алгебраическое разветвленное накрытие плоскости $\mathbb{C P}^{2}$ - это конечное голоморфное отображение $f$ : $X \rightarrow \mathbb{C P}^{2}$ нормальной проективной неприводимой комплексной поверхности $X$ на проективную плоскость. Дивизор ветвления $D \subset X$ - это дивизор якобиана отображения $f$ (кратность $D$ в точке - это локальная степень отображения $f$ минус 1). Кривая ветвления $\bar{H} \subset \mathbb{C P}^{2}$ - это образ носителя дивизора $D$ или, другими словами, множество точек, над которыми $f$ не является локально обратимым отображением.

Как упоминалось во введении, накрытие $f$ определяет монодромию накрытия $\bar{\mu}: \pi_{1}\left(\mathbb{C P}^{2} \backslash \bar{H}, p\right) \rightarrow \mathscr{S}_{N}$ из $\pi_{1}\left(\mathbb{C P}^{2} \backslash \bar{H}, p\right)$ в симметрическую группу $\mathscr{S}_{N}$, $N=\operatorname{deg} f$. Монодромия $\bar{\mu}$ определяется отображением $f$ однозначно с точностью до внутренних автоморфизмов симметрической группы. Обратно, согласно теореме Грауэрта-Ремерта-Штейна [2] гомоморфизм $\bar{\mu}: \pi_{1}\left(\mathbb{C P}^{2} \backslash \bar{H}, p\right) \rightarrow S_{N}$, образ $\operatorname{Im} \bar{\mu}$ которого транзитивно действует на множестве из $N$ элементов, является монодромией некоторого конечного морфизма $f: X \rightarrow \mathbb{C P}^{2}$ степени $\operatorname{deg} f=N$, где $X$ - некоторая нормальная неприводимая проективная алгебраическая поверхность.

Как известно, любая неособая проективная алгебраическая поверхность может быть представлена как конечнолистное накрытие проективной плоскости.

Выбрав “бесконечно удаленную" прямую $R_{\infty} \subset \mathbb{C P}^{2}$, находящуюся в общем положении относительно кривой $\bar{H}$, и положив $\mathbb{C}^{2}=\mathbb{C P}^{2} \backslash R_{\infty}$, получим канонический эпиморфизм $i^{*}: \pi_{1}\left(\mathbb{C}^{2} \backslash H, p\right) \rightarrow \pi_{1}\left(\mathbb{C P}^{2} \backslash \bar{H}, p\right)$, композицию которого $\mu=\bar{\mu} \circ i^{*}$ с монодромией $\bar{\mu}$ также будем называть монодромией накрытия $f$.

Согласно теореме Зариского-ван Кампена фундаментальная группа $\pi_{1}=$ $\pi_{1}\left(\mathbb{C}^{2} \backslash H, p\right)$ является $C$-группой. Ее $C$-порождающие элементы могут быть описаны следующим образом. Рассмотрим произвольную точку $x \in H \backslash \operatorname{Sing} H$ и выберем прямую $\Pi \subset \mathbb{C}^{2}$, пересекающую $H$ трансверсально в точке $x$. Пусть $\Gamma \subset \Pi$ - окружность малого радиуса с центром в $x$. Комплексная ориентация на $\mathbb{C}^{2}$ определяет ориентацию на Г. Пусть $\gamma-$ петля, состоящая из: пути $l$ 
в $\mathbb{C}^{2} \backslash H$, соединяющего точку $p$ с точкой $q \in \Gamma$, окружности $\Gamma$ (с положительной ориентацией) с началом и концом в $q$ и возврата в $p$ вдоль $l$ в обратном направлении. Такие петли $\gamma$ (и соответствующие им элементы в $\pi_{1}$ ) являются $C$-порождающими элементами и называются геометрическими порождающими фундаментальной группы $\pi_{1}=\pi_{1}\left(\mathbb{C}^{2} \backslash H, p\right)$.

Для каждой особой точки $s_{i}$ кривой $H$ выберем малую окрестность $U_{i} \subset \mathbb{C}^{2}$ такую, что $H \cap U_{i}$ задается (в локальных координатах в $U_{i}$ ) нормализованным уравнением Вейерштрасса

$$
w^{k_{i}}+\sum_{j=0}^{k_{i}-1} c_{j}(z) w^{j}=0
$$

(в частности, $w^{2}=z^{3}$, если $s_{i}$ является обыкновенным каспом, и $w^{2}=z^{2}$, если $s_{i}-$ ноуд). Пусть $p_{i}-$ произвольная точка в $U_{i} \backslash \bar{H}$. Напомним, что если $s_{i}-$ обыкновенный касп, то $\pi_{1}\left(U_{i} \backslash H, p_{i}\right)$ изоморфна группе кос $\mathrm{Br}_{3}$ из трех нитей и порождена двумя геометрическими образующими (скажем, $a_{1}$ и $a_{2}$ ), удовлетворяющими соотношению $a_{1} a_{2} a_{1}=a_{2} a_{1} a_{2}$, и если $s_{i}-$ ноуд, то $\pi_{1}\left(U_{i} \backslash H, p_{i}\right)$ изоморфна $\mathbb{Z} \oplus \mathbb{Z}$ и порождена двумя коммутирующими геометрическими образующими.

Выберем гладкие пути $l_{i}$ в $\mathbb{C}^{2} \backslash H$, соединяющие точки $p_{i}$ с $p$. Этот выбор определяет гомоморфизмы $\psi_{i}: \pi_{1}\left(U_{i} \backslash H, p_{i}\right) \rightarrow \pi_{1}$. Группа $\psi_{i}\left(\pi_{1}\left(U_{i} \backslash H, p_{i}\right)\right)=G_{i}$ называется локальной фундаментальной группой особой точки $s_{i}$. Локальные фундаментальные группы $G_{i}$ зависят от выбора путей $l_{i}$ и, следовательно, определены однозначно с точностью до сопряжения в $\pi_{1}$.

Пусть $\operatorname{bmf}(\bar{H})=s=b_{1} \cdot \ldots \cdot b_{n} \in S_{\mathrm{Br}_{m}}-$ брэйд-монодромное разложение на множители кривой $\bar{H}$ и множитель $b_{i}$, соответствующий ее особой точке $s_{i}$, имеет стандартную форму $\bar{b}_{i} \in \mathrm{Br}_{k_{i}, 0}, b_{i}=g_{i}^{-1} \bar{b}_{i} g_{i}$. Обозначим через

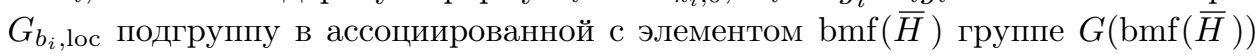
(см. п. 3.4), порожденную элементами $\left(x_{j}\right) g_{i}, j=1, \ldots, k_{i}$. Тогда, ввиду теоремы Зариского-ван Кампена, группа $G_{i}$ совпадает (с точностью до сопряжения) с группой $G_{b_{i}, \text { loc }}$.

8.2. Общие накрытия плоскости. Конечный морфизм $f: X \rightarrow \mathbb{C P}^{2}$, $\operatorname{deg} f=N$, разветвленный над неприводимой (обыкновенной) каспидальной кривой $\bar{H}$, называется общим накрытием плоскости с дискриминантной кривой $\bar{H}$, если монодромия $\mu$ отображения $f$ является общим гомоморфизмом, т. е. удовлетворяет следующим условиям:

(i) $\mu$ - эпиморфизм;

(ii) образ $\mu(\gamma)$ каждой геометрической образующей $\gamma$ является транспозицией в $\mathscr{S}_{N}$;

(iii) образы $\mu\left(G_{i}\right)$ локальных фундаментальных групп $G_{i}$, соответствующих особым точкам кривой $\bar{H}$, являются подгруппами в $\mathscr{S}_{N}$ порядков больше, чем 2.

Последнее условие эквивалентно тому, что $X$ является неособой поверхностью (см., например, [24]). Отметим, что если $\bar{H}$ является дискриминантной кривой некоторого общего накрытия, то ее степень является четным числом, $\operatorname{deg} \bar{H}=2 d$. Для общего накрытия $f$ полный прообраз $f^{*}(\bar{H})$ распадается 
(если $\operatorname{deg} f>2$ ) в сумму $f^{*}(\bar{H})=2 D+C$, где $D \subset X-$ дивизор ветвления отображения $f$, имеющий кратность ветвления, равную двум, и $C$ - приведенная кривая, в которой $f$ не разветвлено. Кривая $D$ неособа и ограничение $f_{\mid D}$ на $D$ является морфизмом степени 1 .

Если $\bar{H}$ - дискриминантная кривая общего накрытия $f: X \rightarrow \mathbb{C P}^{2}$, то квадрат канонического класса $K_{X}^{2}$ поверхности $X$ и ее топологическая эйлерова характеристика $e(X)$ связаны с инвариантами дискриминантной кривой $\bar{H}$ следующими формулами (см. [52]):

$$
\begin{gathered}
K_{S}^{2}=9 \operatorname{deg} f+2 d^{2}-12 d-c-n=9 \operatorname{deg} f-9 d+g-1, \\
e(S)=3 \operatorname{deg} f+4 d^{2}-6 d-3 c-2 n=3 \operatorname{deg} f+2(g-1)-c,
\end{gathered}
$$

где $2 d$ - степень кривой $\bar{H}, g$ - геометрический род ее нормализации, $c$ - число каспов и $n$ - число ноудов.

Как известно, для каждой неособой проективной поверхности $X$, вложенной в проективное пространство $\mathbb{C P}^{r}$, существует (быть может, после вложения Веронезе $\mathbb{C P}^{r} \hookrightarrow \mathbb{C P}^{(r+1)(r+2) / 2}$ с помощью однородных форм степени 2) линейная проекция $\mathrm{pr}: \mathbb{C P}^{r} \rightarrow \mathbb{C P}^{2}$, ограничение которой на $X$ является общим накрытием плоскости. Такие накрытия будем называть общими проекциями на проективную плоскость.

8.3. Гипотеза Кизини. Гипотеза Кизини [53] утверждает, что если общее накрытие $f: X \rightarrow \mathbb{C P}^{2}$ имеет степень $\operatorname{deg} f \geqslant 5$, то $f$ определяется своей дискриминантной кривой однозначно с точностью до изоморфизма поверхности $X$.

Гипотеза Кизини эквивалентна следующему утверждению: если для каспидалъной плоской кривой $\bar{H}$ существуют два общих гомоморфизма $\mu_{i}: \pi_{1}\left(\mathbb{C P}^{2} \backslash \bar{H}\right) \rightarrow \mathscr{S}_{N_{i}}, i=1,2, u N_{1} \geqslant 5$, то $N_{1}=N_{2}$ u $\mu_{2}=\psi \circ \mu_{2}$, где $\psi: \mathscr{S}_{N_{1}} \rightarrow \mathscr{S}_{N_{1}}-$ некоторый внутренний автоморфизм группы $\mathscr{S}_{N_{1}}$.

Используя теорему Ходжа об индексе, в [52] было доказано, что гипотеза Кизини верна для дискриминантной кривой $\bar{H}$ общего накрытия $f: X \rightarrow \mathbb{C P}^{2}$, если

$$
\operatorname{deg} f>\frac{4(3 d+g-1)}{2(3 d+g-1)-c} .
$$

Как было замечено в [54], из неравенства Богомолова-Мияоки-Яо следует, что значения, которые может принимать правая часть неравенства (22), меньше 12 , другими словами, гипотеза Кизини верна для дискриминантных кривых общих накрытий, степень которых больше 11. Кроме того, в [54] было также показано, что если $X$ является поверхностью не общего типа, то гипотеза Кизини верна для дискриминантных кривых общих накрытий $f: X \rightarrow \mathbb{C P}^{2}$, если $\operatorname{deg} f \geqslant 8$.

Условие $\operatorname{deg} f \geqslant 5$ является существенным в гипотезе Кизини. Так, например, кривая $\bar{H} \subset \mathbb{C P}^{2}$ степени 6 с девятью обыкновенными каспами является дискриминантной кривой четырех не эквивалентных общих накрытий проективной плоскости: три из них имеют степень четыре, а четвертое - степень три (см. [55]).

В работе [56] доказана следующая теорема. 
Теорема 8.1. Пусть $f: X \rightarrow \mathbb{C P}^{2}$ является общей проекиией. Тогда общее накрытие $f$ однозначно с точностью до изоморфизма поверхности $X$ определяется своей дискриминантной кривой $\bar{H} \subset \mathbb{C P}^{2}$ за исключением случая, когда $X \simeq \mathbb{C P}^{2}$ вложена в $\mathbb{C P}^{5}$ с помощью многочленов степени два (вложение Веронезе плоскости $\mathbb{C P}^{2}$ в $\left.\mathbb{C P}^{5}\right)$ и $f$ является ограничением на поверхность $X$ линейной проекции $\mathrm{pr}: \mathbb{C P}^{5} \rightarrow \mathbb{C P}^{2}$.

\section{4. Деформации комплексных структур на проективных алгебра-} ических поверхностях. Множество плоских кривых степени $2 d$ естественным образом параметризуется точками пространства $\mathbb{C} \mathbb{P}^{d(2 d+3)}$ (координаты в котором - это коэффициенты уравнения кривой). Подмножество плоских неприводимых кривых степени $2 d$ и рода $g$, имеющих $c$ обыкновенных каспов и соответствующее количество ноудов в качестве своих особых точек, является квазипроективным подмногообразием $\mathscr{M}(2 d, g, c) \subset \mathbb{C P}^{d(2 d+3)}$ (см., например, [57]). Отметим, что если две неособые точки, принадлежащие одной и той же неприводимой компоненте пространства $\mathscr{M}_{\text {red }}(2 d, g, c)$, соответствуют кривым $\bar{H}_{1}$ и $\bar{H}_{2}$, то пары $\left(\mathbb{C P}^{2}, \bar{H}_{1}\right)$ и $\left(\mathbb{C P}^{2}, \bar{H}_{2}\right)$ являются симплектически изотопными. В частности, в этом случае фундаментальные группы $\pi_{1}\left(\mathbb{C P}^{2} \backslash \bar{H}_{1}\right)$ и $\pi_{1}\left(\mathbb{C P}^{2} \backslash \bar{H}_{2}\right)$ изоморфны. Следовательно, если $\bar{H}_{1}$ является дискриминантной кривой некоторого общего накрытия, то $\bar{H}_{2}$ также является дискриминантной кривой некоторого общего накрытия, и если степень накрытия больше 11, то это накрытие единственно.

Если $X \subset \mathbb{C P}^{r}$ - неособая проективная поверхность, то линейные проекции $f: X \rightarrow \mathbb{C P}^{2}$ определяются точками из грассманиана $\mathrm{Gr}_{r+1, r-2}$ (центрами проекций). Множеству общих проекций соответствует некоторое открытое по Зарискому множество $U$ в $\mathrm{Gr}_{r+1, r-2}$. Непрерывная вариация точки в $U$ дает непрерывное семейство общих проекций поверхности $X$, дискриминантные кривые которых принадлежат непрерывному семейству эквисингулярных плоских каспидальных кривых. Следовательно, дискриминантные кривые двух общих проекций поверхности $X \subset \mathbb{C P}^{r}$ принадлежат одной и той же неприводимой компоненте пространства $\mathscr{M}(2 d, g, c)$.

Если $X$ - поверхность общего типа, то согласно теореме Бомбиери при $m \geqslant 5$ сечения $m$-канонического класса $m K_{X}$ задают вложение (по модулю $(-2)$-кривых) поверхности $X$ в проективное пространство (т. е. образ поверхности $X-$ это нормальная поверхность, полученная из $X$ стягиванием в особые точки всех неособых рациональных кривых, индекс самопересечения которых равен -2). Пусть две поверхности $X_{1}$ и $X_{2}$ общего типа с обильным каноническим классом, инварианты которых, $K_{X}^{2}=k$ и арифметический род $p_{a}=p$, одинаковы, вложены с помощью $m$-канонического класса в одно и то же проективное пространство $\mathbb{C P}^{r}$ и принадлежат одной и той же неприводимой компоненте грубого пространства модулей $\mathscr{M}_{X}(k, p)$ поверхностей [58], тогда общие проекции $f_{1}$ поверхности $X_{1}$ и $f_{2}$ поверхности $X_{2}, \operatorname{deg} f_{i}=m^{2} k$, принадлежат одному и тому же непрерывному семейству общих проекций. Следовательно, для таких общих проекций $f_{1}$ и $f_{2}$ их дискриминантные кривые (которые будем называть m-каноническими дискриминантными кривыми) принадлежат одной и той же неприводимой компоненте пространства $\mathscr{M}(2 d, g, c)$ (согласно формулам (21) 
тройка чисел $(m, k, p)$ и инварианты $(2 d, g, c)$ m-канонической дискриминантной кривой однозначно определяются друг другом). Следовательно, в силу теоремы 8.1 индуцированное отображение из множества неприводимых компонент пространства $\mathscr{M}_{X}(k, p)$ в множество неприводимых компонент пространства $\mathscr{M}(2 d, g, c)$ является вложением. Таким образом, инварианты, различающие неприводимые компоненты пространства $\mathscr{M}(2 d, g, c)$, различают неприводимые компоненты пространства модулей $\mathscr{M}_{X}(k, p)$.

Отсюда следует, что брэйд-монодромный тип $\beta_{\text {can }, m}(X)=\operatorname{bmt}\left(\bar{H}_{m}\right)$ относительно общей проекции на проективную прямую $m$-канонической дискриминантной кривой $\bar{H}_{m}$ при $m \geqslant 5$ является инвариантом неприводимых компонент пространства модулей $\mathscr{M}_{X}(k, p)$ поверхностей общего типа с обильным каноническим классом (здесь $\operatorname{bmt}\left(\bar{H}_{m}\right)$ - это брэйд-монодромный тип кривой $\bar{H}_{m}$ относительно общей проекции на проективную прямую). Как показывает следующий пример, этот инвариант должен, по всей видимости, различать, по крайней мере, разные связные компоненты пространства модулей поверхностей общего типа.

ПримеР 8.1. Пусть $X$ - поверхность общего типа геометрического рода нуль с $K_{X}^{2}=9$. Так как топологическая эйлерова характеристика $e(X)=3$, то $X$ является поверхностью Мияоки-Яо, и, следовательно, универсальным накрытием поверхности $X$ является шар в $\mathbb{C}^{2}$. Поэтому согласно [59] любой гомеоморфизм $h$ поверхности $X$ гомотопен голоморфному либо антиголоморфному автоморфизму поверхности $X$. С другой стороны, в [60] было показано, что поверхности общего типа геометрического рода нуль с $K_{X}^{2}=9$ не имеют антиголоморфных автоморфизмов. Следовательно, любой гомеоморфизм поверхности $X$ гомотопен голоморфному автоморфизму. Кроме того, отсюда следует, что если $X=(M, J)$, где $M$ - подлежащее вещественное многообразие, а $J$ - комплексная структура на $X$, то комплексное многообразие $\bar{X}=(M,-J)$ не изоморфно многообразию $X$ и, следовательно, поверхности $X$ и $\bar{X}$ принадлежат разным неприводимым компонентам пространства модулей поверхностей общего типа (так как $X$ и $\bar{X}$ - жесткие поверхности, то соответствующие компоненты нульмерны). Пусть $\bar{H}_{m, 1} \subset \mathbb{C P}^{2}-m$-каноническая дискриминантная кривая общего накрытия $f_{m}: X \rightarrow \mathbb{C P}^{2}$, заданного сечениями $m$-канонического класса. Рассмотрим стандартное комплексное сопряжение $c: \mathbb{C P}^{2} \rightarrow \mathbb{C P}^{2}$, заданное в однородных координатах формулой $c^{*}\left(x_{i}\right)=\bar{x}_{i}$. Композиция $\bar{f}_{m}=c \circ f_{m}: \bar{X}_{m} \rightarrow \mathbb{C P}^{2}$ также является $m$-каноническим общим накрытием с дискриминантной кривой $\bar{H}_{m, 2}=c\left(\bar{H}_{m, 1}\right)$. Отметим, что пары $\left(\mathbb{C P}^{2}, H_{m, 1}\right)$ и $\left(\mathbb{C P}^{2}, H_{m, 2}\right)$ являются диффеоморфными. С другой стороны, имеем $\bar{f}_{m, 2}^{*}([L])=m\left[K_{\bar{X}}\right]=-m\left[K_{X}\right] \in H^{2}(X, \mathbb{Z})$, где $L-$ прямая в $\mathbb{C P}^{2}$, и легко видеть, что если бы кривые $\bar{H}_{m, 1}$ и $\bar{H}_{m, 2}$ были изотопны, то ввиду единственности общего гомоморфизма из $\pi_{1}\left(\mathbb{C P}^{2} \backslash \bar{H}_{m, 1}\right) \simeq \pi_{1}\left(\mathbb{C P}^{2} \backslash \bar{H}_{m, 2}\right)$ в симметрические группы (теорема 8.1) эта изотопия индуцировала бы гомеоморфизм поверхностей $h: X \rightarrow \bar{X}$ такой, что $h^{*}\left(\left[K_{X}\right]\right)=-\left[K_{X}\right]$. Следовательно, он должен был бы быть гомотопен антиголоморфному автоморфизму поверхности $X$. Но это невозможно ввиду отсутствия у $X$ антиголоморфных автоморфизмов. Отсюда и из теоремы 5.1 следует, что $\operatorname{bmt}\left(\bar{H}_{m, 1}\right) \neq \operatorname{bmt}\left(\bar{H}_{m, 2}\right)$. 
Как отмечалось выше, образ $X_{m}$ поверхности общего типа $X$ при $m$-каноническом отображении в проективное пространство может стать особым, если на $X$ лежат $(-2)$-кривые. Особые точки поверхности $X_{m}$ - это особые точки типов $A_{n}, n \geqslant 1, D_{n}, n \geqslant 4, E_{6}, E_{7}$ и $E_{8}$, локальные уравнения которых:

$$
\begin{aligned}
A_{n}: & z^{2}=v^{2}-u^{n+1} & (n \geqslant 1), \\
D_{n}: & z^{2}=(v-2 u)\left(v^{2}-u^{n-2}\right) & (n \geqslant 4), \\
E_{6}: & z^{2}=v^{3}-u^{4}, & \\
E_{7}: & z^{2}=v\left(v^{2}-u^{3}\right), & \\
E_{8}: & z^{2}=v^{3}-u^{5} . &
\end{aligned}
$$

Общая линейная проекция $f_{m}: X_{m} \rightarrow \mathbb{C P}^{2}$ разветвлена над алгебраической кривой $\bar{H}_{m}$. В окрестности особой точки $s$ поверхности $X_{m}$ накрытие $f_{m}$ изоморфно проекции на плоскость с координатами $u, v$ поверхности $z^{2}=h(u, v)$, где $z^{2}=h(u, v)$ - уравнение одной из $A$-, $D$ - или $E$-особенностей. Точке $s$ соответствует особая точка $о$ кривой $\bar{H}$ такого же типа, что и точка $s$. На кривой $\bar{H}_{m}$ кроме особых точек, произошедших из особых точек поверхности $X_{m}$, которые мы будем называть $s$-точками, имеются еще дополнительные особые точки типа $A_{1}$ (ноуды) и $A_{2}$ (каспы), которые мы будем называть $p$-ноудами и $p$-каспами. Обозначим через $2 d$ степень кривой $\bar{H}_{m}$ (это четное число).

Монодромия $\bar{\mu}_{m}: \pi_{1}\left(\mathbb{C P}^{2} \backslash \bar{H}\right) \rightarrow \mathscr{S}_{N}$, где $N=m^{2} K_{X}^{2}$, обладает следующими свойствами:

(i) $\bar{\mu}_{m}$ является эпиморфизмом;

(ii) образ $\bar{\mu}_{m}(\gamma)$ каждой геометрической образующей $\gamma$ является транспозицией в $\mathscr{S}_{N}$;

(iii) образы $\bar{\mu}_{m}\left(G_{i}\right)$ локальных фундаментальных групп $G_{i}$, соответствующих особым точкам $o_{i}$ кривой $\bar{H}$, являются подгруппами в $\mathscr{S}_{N}$ порядков больше чем 2 , если $o_{i}$ являются р-точками, и подгруппами порядка 2 , если $o_{i}$ являются s-точками.

Применяя обобщение неравенства $(22)$ к общим накрытиям плоскости поверхностями с $A$-, $D$ - или $E$-особенностями, заданным кратным каноническим классом (см. [61]), как и в неособом случае, можно показать, что индуцированное отображение из множества связных компонент пространства $\mathscr{M}_{X}(k, p)$ в множество связных компонент пространства $\mathscr{M}(2 d, \geqslant c, \geqslant n)$ также является вложением, где $\mathscr{M}(2 d, \geqslant c, \geqslant n)$ - подпространство плоских неприводимых кривых степени $2 d$, имеющих по крайней мере $c$ обыкновенных каспов и $n$ ноудов, а также некоторое количество других $A$-, $D$ - или $E$-особых точек в качестве своих особенностей. Отсюда следует, что если $\operatorname{bmf}(\bar{H})=s_{p} \cdot s_{s}$, где $s_{s}=b_{1} \cdot \ldots \cdot b_{l}-$ произведение всех локальных монодромий s-точек кривой $\bar{H}_{m}$, то брэйд-монодромный тип $\beta_{\mathrm{can}, m}(X)$ элемента $s_{p} \cdot r\left(s_{s}\right)$ (т. е. его орбита при действии $\mathrm{Br}_{\operatorname{deg} \bar{H}_{m}}$ на $S_{\mathrm{Br}_{\operatorname{deg}} \bar{H}_{m}}$ ) является инвариантом связной компоненты пространства модулей поверхностей общего типа, которой принадлежит поверхность $X$. Здесь $r\left(s_{s}\right)$ - это регенерация элемента $s_{s}$, полученная заменой каждого множителя $b_{i}=g_{i} \bar{b}_{i} g_{i}^{-1}$ произведением $\left(g_{i} a_{j_{1}(i)} g_{i}^{-1}\right) \cdot \ldots \cdot\left(g_{i} a_{j_{n_{i}}(i)} g_{i}^{-1}\right)$, и $\bar{b}_{i}=a_{j_{1}(i)} \ldots a_{j_{n_{i}}(i)} \in \mathrm{Br}_{k}^{+}-$стандартная форма брэйд-монодромии особой точки $o_{i}$ кратности $k$. Отметим, что $s_{p} \cdot r\left(s_{s}\right)$ является разложением на множители 
брэйд-монодромии кривой Гурвица, полученной из кривой $\bar{H}_{m}$ в результате сглаживания всех еe s-точек.

ГИПОТЕЗА 8.1. Пусть поверхности общего типа $X_{1}$ u $X_{2}$ принадлежат разным связным компонентам пространства модулей поверхностей общего muna. Тогда $\beta_{\text {can }, m}\left(X_{1}\right) \neq \beta_{\text {can }, m}\left(X_{2}\right)$ nрu $m \geqslant 5$.

Эта гипотеза показывает важность решения следующих проблем.

Проблема 8.1. Разрешима ли проблема слов в полугруппе $S_{A_{0} \cup A_{1} \cup A_{2}}$ ?

ПроБЛЕма 8.2. Существует ли конечный алгоритм для решения вопроса о том, являются ли два элемента $s_{1}, s_{2} \in S_{A_{0} \cup A_{1} \cup A_{2}}$ сопряженными в этой nолугруппе (m.е. существует ли $b \in \mathrm{Br}_{m}$ такой, что $\left.s_{2}=\lambda(b)\left(s_{1}\right)\right)$ ?

\section{§ 9. Конечнолистные симплектические накрытия проективной плоскости}

\section{1. Построение конечнолистных накрытий проективной плоско-} сти, разветвленных вдоль кривых Гурвица. Пусть кривая Гурвица $\bar{H} \subset \mathbb{C P}^{2}$ задана как множество нулей сечения расслоения $\mathscr{L}(m)$ (см. п. 5.4), которое удовлетворяет условиям, описанным в лемме 5.1. Рассмотрим гомоморфизм $\mu: \pi_{1} \rightarrow \mathscr{S}_{N}$ из фундаментальной группы $\pi_{1}=\pi_{1}\left(\mathbb{C}^{2} \backslash H\right)$ дополнения к аффинной кривой Гурвица $H=\left(\mathbb{C P}^{2} \backslash R_{\infty}\right) \cap \bar{H}$ в симметрическую группу $\mathscr{S}_{N}$ такой, что его образ $\operatorname{Im} \mu$ действует транзитивно на множестве, состоящем из $N$ элементов.

Пусть $s_{i}$ - отрицательный ноуд кривой $H$. Локальная фундаментальная группа $G_{i}$ точки $s_{i}$ порождается двумя коммутирующими геометрическими порождающими элементами, скажем, $\gamma_{i, 1}$ и $\gamma_{i, 2}$. Обозначим $N_{i, j}=\{1 \leqslant n \leqslant N$ | $\left.\mu\left(\gamma_{i, j}\right)(n) \neq n\right\}$. Скажем, что гомоморфизм $\mu$ является хорошим в отрицательном ноуде $s_{i}$, если $N_{i, 1} \cap N_{i, 2}=\varnothing$. Гомоморфизм $\mu$ называется монодромией cmenени $N$, если он является хорошим во всех отрицательных ноудах.

Гомоморфизм $\mu$ определяет неразветвленное накрытие $f=f_{\mu}: X \rightarrow \mathbb{C}^{2} \backslash H$ степени $N$. Это накрытие может быть продолжено до конечного разветвленного накрытия $\tilde{f}: \widetilde{X} \rightarrow \mathbb{C P}^{2}$ с ветвлением вдоль $\bar{H}$ и, возможно, вдоль $R_{\infty}$.

Для того чтобы описать это продолжение, рассмотрим одну из геометрических порождающих $\gamma$ с центром в точке $p \in H \backslash$ Crit $H$, где Crit $H$ - множество критических точек кривой $H$. Образ $\mu(\gamma)$ в $\mathscr{S}_{N}$ является произведением циклических перестановок $\sigma_{1}, \ldots, \sigma_{n_{p}}$ (возможно, что порядки некоторых из этих перестановок равны единице). Каждая из этих перестановок $\sigma_{l}$ циклически переставляет элементы в множестве $\left\{n_{1, l}, \ldots, n_{r_{l}, l}\right\}, 1 \leqslant n_{j, l} \leqslant N$, где $r_{l}-$ порядок перестановки. Тогда число прообразов $\tilde{f}^{-1}(p)$ равно $n_{p}$ и каждая точка $q$ из $\tilde{f}^{-1}(p)$ соответствует некоторой циклической перестановке $\sigma_{l}$. Вблизи точки $q_{l}$, соответствующей циклической перестановке $\sigma_{l}$, накрытие $\tilde{f}$ является циклическим накрытием степени $r_{l}$, разветвленным вдоль $H$ и локально изоморфным подмногообразию в $\mathbb{C}^{3}$, заданному уравнением $w^{r_{l}}=v-v_{j}(u)$, где $v-v_{j}(u)=0-$ локальное уравнение кривой $\bar{H}$ в точке $p$. Эти локальные изоморфизмы определяют на $\widetilde{X}$ структуру гладкого многообразия в каждой точке $q$, лежащей над точкой $p \in H \backslash$ Crit $H$. 
Пусть $s_{i} \in$ Crit $H$ является отрицательным ноудом. Как было упомянуто выше, локальная фундаментальная группа $G_{i}$ порождается двумя геометрическими порождающими элементами $\gamma_{i, 1}$ и $\gamma_{i, 2}$. Образы $\mu\left(\gamma_{i, j}\right)$ в $\mathscr{S}_{N}$ являются произведениями циклических перестановок $\sigma_{1, i, j}, \ldots, \sigma_{k_{i, j}, i, j}$. Пусть $\sigma_{l, i, j}=\left(n_{1, l, i, j}, \ldots, n_{r_{l, i, j}, l, i, j}\right)-$ перестановка порядка $r_{l, i, j}$. Положим $N_{i, 1,2}=$ $\left\{1 \leqslant n \leqslant N \mid \mu\left(\gamma_{i, 1}\right)(n)=n\right.$ и $\left.\mu\left(\Gamma_{i, 2}\right)(n)=n\right\}$. Так как $\mu$ является монодромией, множество $\tilde{f}^{-1}\left(s_{i}\right)$ находится во взаимно однозначном соответствии с объединением всех циклических перестановок $\sigma_{l, i, j}, j=1,2$, имеющих порядки больше единицы, и множества $N_{i, 1,2}$. Более того, если $q \in \tilde{f}^{-1}\left(s_{i}\right)$ соответствует элементу из $N_{i, 1,2}$, то $\tilde{f}$ является изоморфизмом некоторой окрестности $V$ точки $q$ и ее образа $\tilde{f}(V)$, и если $q \in \tilde{f}^{-1}\left(s_{i}\right)$ соответствует циклической перестановке $\sigma_{l, i, j}$ порядка больше единицы, то ограничение накрывающего отображения $\tilde{f}$ на некоторую окрестность точки $q$ является циклическим накрытием некоторой окрестности точки $s_{i}$ степени $r_{l, i, j}$, разветвленным в $j$-й ветви отрицательного ноуда. Это накрытие локально изоморфно многообразию в $\mathbb{C}^{3}$, заданному уравнением $w^{r_{l, i, j}}=v-v_{j}(u)$, где $v-v_{j}(u)=0$ - локальное уравнение $j$-й ветви кривой $\bar{H}$ в точке $s_{i}$. Эти локальные изоморфизмы определяют на $\widetilde{X}$ структуру гладкого многообразия в каждой точке $q \in \tilde{f}^{-1}\left(s_{i}\right)$.

Пусть $s_{i} \in$ Crit $H$ не является отрицательным ноудом. Тогда над небольшой окрестностью $U$ точки $s_{i}$, в которой кривая $H$ задается аналитическим уравнением, прообраз $\tilde{f}^{-1}(U)$ является несвязным объединением $n_{s_{i}}$ открытых окрестностей, находящихся во взаимно однозначном соответствии с элементами из множества орбит действия группы $\mu\left(G_{i}\right)$ на множестве, состоящем из $N$ элементов. Согласно теореме Грауэрта, Ремерта и Штейна (доказательство см. в [59]), над окрестностью $U$ точки $s_{i}$ многообразие $\widetilde{X}$ может быть снабжено структурой двумерного комплексно-аналитического многообразия.

По предположению в некоторой окрестности $U$ прямой $R_{\infty}$ (где $U=\mathbb{C P}^{2} \backslash$ $B(r)$ и $B(r) \subset \mathbb{C}_{1}^{2}$ - это шар радиуса $\left.r \gg 1\right)$ кривая $\bar{H}$ совпадает с алгебраической кривой $\bar{C} \subset \mathbb{C P}^{2}$ степени $m$, заданной в $U$ уравнением $v_{2}^{m}-1=0$. Если мы выберем базисную точку $p_{0}$ лежащей в $U$, мы можем рассмотреть копредставления Зариского-ван Кампена группы $\pi_{1}$ и фундаментальной группы $\tilde{\pi}_{1}=\pi_{1}\left(\mathbb{C P}^{2} \backslash\left(\bar{C} \cup R_{\infty}\right), p_{0}\right)$, имеющие одни и те же множества порождающих элементов, и легко видеть, что эти представления определяют эпиморфизм $e: \tilde{\pi}_{1} \rightarrow \pi_{1}$. Композиция $\mu$ о е определяет разветвленное накрытие $\tilde{g}: \widetilde{Z} \rightarrow \mathbb{C P}^{2}$ с ветвлением вдоль $\bar{C}$ и, возможно, вдоль $R_{\infty}$. Легко видеть, что накрытия $\tilde{f}$ и $\tilde{g}$ изоморфны над $U$. Следовательно, многообразие $\tilde{f}^{-1}(U)$ может быть отождествлено с $\tilde{g}^{-1}(U)$ с помощью изоморфизма $h: \tilde{f}^{-1}(U) \rightarrow \tilde{g}^{-1}(U)$. Таким образом, мы можем рассматривать $\tilde{f}^{-1}(U)$ как комплексно-аналитическое многообразие.

Понятие симплектической поверхности с особенностями может быть обобщено на произвольную размерность следующим образом.

Пусть $Y$ - неособое проективное комплексное многообразие комплексной размерности $\operatorname{dim}_{\mathbb{C}} Y=n$ и $\omega$ - кэлерова форма на $Y,[\omega] \in H^{2}(Y, \mathbb{Z})$. Рассмотрим $(Y, \omega)$ как симплектическое многообразие, $\operatorname{dim}_{\mathbb{R}} Y=2 n$. Замкнутое подмногообразие $X$ в $Y$ называется симплектическим (под)многообразием с аналитическими особенностями, если существуют открытые подмножества 
$U_{0} \subset U \subset Y$ такие, что замыкание $\bar{U}_{0}$ в $Y$ содержится в $U, X \cap U$ является комплексно-аналитическим подмножеством в $U$ и $X \backslash \bar{U}_{0}$ является гладким симплектическим подмногообразием. Обозначим через $\operatorname{Sing} X$ множество точек из $X$, в которых $X$ не является гладким. Тогда $\operatorname{Sing} X$ является проективным алгебраическим подмногообразием в $Y$.

В [15] была доказана следующая теорема.

Теорема 9.1. Пусть $\bar{H}$ - кривая Гурвица (возможно, с отрицателъными ноудами), $\mu: \pi_{1}\left(\mathbb{C}^{2} \backslash H\right) \rightarrow \mathscr{S}_{N}-$ монодромия $u \tilde{f}: \widetilde{X} \rightarrow \mathbb{C P}^{2}-$ конечное накрытие, ассоциированное с монодромией $\mu$. Тогда $\widetilde{X}$ может быть вложено в некоторое проективное пространство $\mathbb{C P}^{M}$ как симплектическое подмногообразие с аналитическими особенностями и $\tilde{f}$ является ограничением некоторой линейной проекции $\mathrm{pr}: \mathbb{C P}^{M} \rightarrow \mathbb{C P}^{2}$.

Доказательство этой теоремы состоит в том, что для каждой описанной выше окрестности $\tilde{f}^{-1}(U)$ покрытия плоскости $\mathbb{C P}^{2}$ окрестностями $\{U\}$ строится вложение в проективное пространство $\mathbb{C P}^{M}$ при некотором достаточно большом $M$, согласованное на пересечении этих окрестностей, такое, что образ многообразия $\widetilde{X}$ при полученном вложении в $\mathbb{C P}^{M}$ является симплектическим многообразием с аналитическими особенностями.

Кроме того, там же была доказана следующая лемма.

Лемма 9.1. Пусть $X$ - симплектическое многообразие с аналитическими особенностями в комплексном проективном многообразии $Y$ с кэлеровой формой $\omega,[\omega] \in H^{2}(Y, \mathbb{Z})$. Пусть $Z \subset \operatorname{Sing} X$ - неособое проективное подмногообразие в $Y, \sigma: \bar{Y} \rightarrow Y-$ моноидальное преобразование многообразия $Y$ с иентром в $Z$ и $\bar{X}$ - собственный прообраз многообразия $X$. Тогда существует кэлерова форма $\bar{\omega}$ на $\bar{Y},[\bar{\omega}] \in H^{2}(\bar{Y}, \mathbb{Z})$, такая, что $\bar{X}$ является симплектическим многообразием с аналитическими особенностями в $(\bar{Y}, \bar{\omega})$.

Эта лемма и теорема Хиронаки о разрешении аналитических особенностей влекут

СЛЕДСТВИЕ 9.1. Для конечного разветвленного накрытия $\tilde{f}: \widetilde{X} \rightarrow \mathbb{C P}^{2} c$ ветвлением вдоль кривой Гурвица $\bar{H} \subset \mathbb{C P}^{2} u$, возможно, вдоль $R_{\infty}$ существует вложение в некоторое проективное многообразие $Y$ разрешения особенностей $\bar{X}$ симплектического многообразия с аналитическими особенностями $\widetilde{X}$ такое, что $\bar{X}$ является симплектическим подмногообразием в этом проективном многообразии относительно некоторой кэлеровой бормы $\omega$, $[\omega] \in H^{2}(Y, \mathbb{Z})$.

\section{2. Общие симплектические накрытия проективной плоскости.}

Пусть $(X, \omega)$ - компактное симплектическое четырехмерное многообразие с симплектической формой $\omega$, класс когомологий которой $[\omega] \in H^{2}(X, \mathbb{Z})$. Зафиксируем совместимую с $\omega$ почти комплексную структуру $J$ и соответствующую риманову метрику $g$. Пусть $L$ - линейное расслоение на $X$, первый класс Черна которого есть [ $\omega]$. В [3] доказано, что для $k \gg 0$ линейное расслоение $L^{\otimes k}$ имеет много почти голоморфных сечений, так что можно выбрать три из них, которые дают почти голоморфное общее накрытие $f_{k}: X \rightarrow \mathbb{C P}^{2}$ степени $N_{k}=k^{2}[\omega]^{2}$, разветвленное вдоль каспидальной кривой Гурвица $\bar{H}$ (возможно, 
с отрицательными ноудами). Накрытия $f_{k}$ локально ведут себя так же, как общие проекции в алгебраическом случае.

Обратно, если заданы каспидальная неприводимая кривая Гурвица $\bar{H} \subset \mathbb{C P}^{2}$ (возможно, с отрицательными ноудами) и общий гомоморфизм (монодромия) $\bar{\mu}$ из фундаментальной группы ее дополнения в симметрическую группу $\mathscr{S}_{N}$, то согласно теореме 9.1 можно построить гладкое накрытие $f: X \rightarrow \mathbb{C P}^{2}$ степени $N$, разветвленное над $\bar{H}$, причем $X$ вложено в некоторое проективное пространство $\mathbb{C P}^{M}$ как симплектическое подмногообразие $(X, \omega)$ с симплектической формой $\omega$, являющейся ограничением формы Фубини-Штуди, а $f$ является ограничением на $X$ некоторой линейной проекции и $[\omega]=f^{*}\left(c_{1}(R)\right) \in$ $H^{2}(X, \mathbb{Z})$, где $c_{1}(R)$ - это класс когомологий, двойственный прямой $R \in \mathbb{C P}^{2}$.

В алгебраическом случае если $(X, L)$ - поляризованная проективная поверхность, где $L$ - обильное линейное расслоение на $X$, то для достаточно большого $k$ сечения расслоения $L^{\otimes k}$ определяют вложение поверхности $X$ в некоторое проективное пространство $\mathbb{C P}^{r}$. Ограничение общей проекции $\mathbb{C P}^{r} \rightarrow \mathbb{C P}^{2}$ на $X$ дает общее накрытие $f_{k}: X \rightarrow \mathbb{C P}^{2}$, разветвленное в некоторой каспидальной кривой $\bar{H}_{k}$, степень накрытия равна $k^{2} c_{1}(L)^{2}$.

Поляризация определяет канонически симплектическую структуру $\omega_{L}$ на $X$. Для ее определения выберем $k \gg 0$ и вложим $X$ в проективное пространство $\mathbb{C P}^{r}$ с помощью сечений расслоения $L^{\otimes k}$. Тогда $\omega_{L}-$ это ограничение симплектической формы $\frac{1}{k} \omega_{0}$ на $X$, где $\omega_{0}$ - симплектическая форма ФубиниШтуди на $\mathbb{C P}^{r}$. Форма $\omega_{L}$ является положительной $(1,1)$-формой, представляющей первый класс Черна $c_{1}(L) \in H^{2}(X, \mathbb{Z})$. Если $X$ и $X^{\prime}$ принадлежат одному и тому же алгебраическому связному семейству неособых поверхностей в $\mathbb{C P}^{r}$, то согласно теореме Мозера деформация поверхности $X$, определяемая этим семейством, задает симплектоморфизм симплектических многообразий $\left(X, \omega_{L}\right)$ и $\left(X^{\prime}, \omega_{L^{\prime}}\right)$.

Назовем $k$-м брэйд-монодромным инвариантом $\beta_{L, k}(X, L)$ поляризованной комплексной поверхности $(X, L)$ тип брэйд-монодромного разложения на множители кривой $\bar{H}_{k}$.

Теорема 9.2. Пусть $(X, L)$ u $\left(X^{\prime}, L^{\prime}\right)$ - две поляризованные проективные поверхности. Предположим, что $\beta_{L, k}(X, L)=\beta_{L^{\prime}, k}\left(X^{\prime}, L^{\prime}\right)$ для некоторого $k$ такого, что $k^{2} c_{1}(L)^{2} \geqslant 12$. Тогда многообразия $\left(X, \omega_{L}\right)$ u $\left(X^{\prime}, \omega_{L^{\prime}}\right)$ симплектоморфньь.

ДокАЗАТЕЛЬСтво. По предположению кривые ветвления $\bar{H}$ и $\bar{H}^{\prime}$ общих проекций на $\mathbb{C P}^{2}$, определенных соответственно сечениями расслоений $L^{\otimes k}$ и $\left(L^{\prime}\right)^{\otimes k}$, имеют один и тот же тип разложений брэйд-монодромии на множители. Таким образом, ввиду теоремы 5.1, после перемасштабирования, можно показать, что кривые $\bar{H}$ и $\bar{H}^{\prime}$ становятся симплектически изотопными как каспидальные кривые Гурвица. Из теоремы 8.1 следует, что существует единственная общая монодромия $\mu: \pi_{1}\left(\mathbb{C P}^{2} \backslash \bar{H}\right) \rightarrow \mathscr{S}_{N}$ для $N=k^{2} c_{1}\left(L_{1}\right)^{2}$. Следовательно, имеем семейство $f_{t}: X \times[0,1] \rightarrow \mathbb{C P}^{2} \times[0,1]$ разветвленных накрытий плоскости $\mathbb{C P}^{2}$, связывающее многообразия $X$ и $X^{\prime}$. Вложив $\mathbb{C P}^{r}$ в некоторое пространство $\mathbb{C P}^{M}, M \gg r$, как линейное подпространство и применив конструкцию, использованную в доказательстве теоремы 8.1 (см. [15]), можно построить вложение семейства $X \times[0,1]$ в $\mathbb{C P}^{M}$ такое, что $X_{t}=X \times\{t\}$ 
является симплектическим подмногообразием в $\mathbb{C P}^{M}$, а $f_{t}$ является ограничением на $X_{t}$ линейной проекции $\mathbb{C P}^{M} \rightarrow \mathbb{C P}^{2}$ при каждом $t$. Для завершения доказательства теоремы осталось применить теорему Мозера.

Из доказательства теоремы 9.2 видно, что теорема 8.1 играет существенную роль для применения брэйд-монодромных разложений на множители дискриминантных кривых общих проекций в качестве инвариантов симплектических структур на четырехмерных многообразиях. Однако доказательство теоремы 8.1 существенно использует теорему Ходжа об индексе и неравенство Богомолова-Мияоки-Яо для алгебраических поверхностей. Аналоги этих теорем неизвестны в симплектическом случае, что не позволяет обобщить теорему 8.1 на общие накрытия, разветвленные вдоль каспидальных кривых Гурвица.

Поэтому, чтобы сформулировать критерий симплектоморфности симплектических накрытий проективной плоскости, нам понадобится не только разложение на множители брэйд-монодромии дискриминантной кривой общего накрытия, приходится также учитывать монодромию накрытия, т. е. нам понадобится понятие слабой $\mu$-эквивалентности пар $\left(s_{1}, \mu_{s_{1}}\right)$ и $\left(s_{2}, \mu_{s_{2}}\right)$, где $\left(s_{i}, \mu_{s_{i}}\right)$ - пара, состоящая из элемента $s_{i} \in S_{\mathrm{Br}_{m}}$ и гомоморфизма $\mu_{s_{i}}: G\left(s_{i}\right) \rightarrow \mathscr{S}_{N}$ в симметрическую группу $\mathscr{S}_{N}$ для некоторого $N$ (здесь $G(s)$ - это группа, ассоциированная с элементом $s$, см. $\S 4)$.

Скажем, что две пары $\left(s_{1}, \mu_{s_{1}}\right)$ и $\left(s_{2}, \mu_{s_{2}}\right)$ являются эквивалентными, если $s_{1}$ и $s_{2}$ принадлежат одной и той же орбите при действии сопряжением группы $\mathrm{Br}_{m}$ на $S_{\mathrm{Br}_{m}}$ (т. е. существует элемент $b \in \mathrm{Br}_{m}$ такой, что $\left.s_{2}=\lambda(b)\left(s_{1}\right)\right)$ и $\mu_{2}=\mu_{1} \circ \gamma_{b}$, где $\gamma_{b}-$ изоморфизм, который был определен в предложении 3.1 .

Говорят, что две пары $\left(s_{1}, \mu_{s_{1}}\right)$ и $\left(s_{2}, \mu_{s_{2}}\right)$ получены друг из друга с помощью допустимого преобразования: $\left(s_{1}, \mu_{s_{1}}\right) \leftrightarrow\left(s_{2}, \mu_{s_{2}}\right)$, если существует такой элемент $g \in A_{1}$, что $s_{2}=g \cdot\left(g^{-1}\right) \cdot s_{1}, \mu_{1}=\psi_{g \cdot\left(g^{-1}\right)} \circ \mu_{2}$ и порядок группы $\mu_{s_{2}}\left(G_{g, \text { loc }}\right)$ больше двух, где $\psi_{g \cdot\left(g^{-1}\right)}$ - эпиморфизм, который был определен в предложении 3.1. Скажем, что две пары $\left(s^{\prime}, \mu_{s^{\prime}}\right)$ и $\left(s^{\prime \prime}, \mu_{s^{\prime \prime}}\right)$ являются слабо $\mu$-эквивалентными, если существует последовательность пар $\left(s_{1}, \mu_{1}\right), \ldots$, $\left(s_{n}, \mu_{n}\right)$ такая, что $\left(s^{\prime}, \mu_{s^{\prime}}\right)=\left(s_{1}, \mu_{1}\right),\left(s^{\prime \prime}, \mu_{s^{\prime \prime}}\right)=\left(s_{n}, \mu_{n}\right)$ и для каждого $i=$ $1, \ldots, n-1$ пары $\left(s_{i}, \mu_{i}\right)$ и $\left(s_{i+1}, \mu_{i+1}\right)$ либо эквивалентны, либо получены друг из друга с помощью допустимого преобразования.

Приведем еще одно эквивалентное определение слабой $\mu$-эквивалентности. Для этого свяжем следующим образом с каждой парой $\left(s, \mu_{s}\right)$ группу $\widetilde{G}(s)$ и эпиморфизм $\tilde{\mu}_{s}: \widetilde{G}(s) \rightarrow \mathscr{S}_{N}$. Скажем, что пара $\left(\gamma_{i}, \gamma_{j}\right) C$-порождающих элементов группы $G(s)$ слабо коммутирует в $G(s)$, если $\mu_{s}\left(\gamma_{i}\right)$ и $\mu_{s}\left(\gamma_{j}\right)$ являются не равными между собой коммутирующими транспозициями в $\mathscr{S}_{N}$. Положим $\widetilde{G}(s)=G(s) / N$, где $N$ - это нормальная подгруппа в $G(s)$, нормально порожденная коммутаторами $\left[\gamma_{i}, \gamma_{j}\right]$ всех слабо коммутирующих пар $C$-порождающих элементов группы $G(s)$. Пусть $c: G(s) \rightarrow \widetilde{G}(s)$ - канонический эпиморфизм. Эпиморфизм $\mu_{s}$ индуцирует эпиморфизм $\tilde{\mu}_{s}: \widetilde{G}(s) \rightarrow \mathscr{S}_{N}$ такой, что $\mu_{s}=\tilde{\mu}_{s} \circ c$. Легко видеть, что две пары $\left(s^{\prime}, \mu_{s^{\prime}}\right)$ и $\left(s^{\prime \prime}, \mu_{s^{\prime \prime}}\right)$ являются слабо $\mu$-эквивалентными, если элементы $s^{\prime}$ и $s^{\prime \prime}$ являются слабо эквивалентными и существует такой $C$-изоморфизм $\varphi: \widetilde{G}\left(s^{\prime}\right) \rightarrow \widetilde{G}\left(s^{\prime \prime}\right)$, что $\tilde{\mu}_{s^{\prime}}=\tilde{\mu}_{s^{\prime \prime}} \circ \varphi$.

Рассмотрим два общих накрытия $f^{\prime}: X^{\prime} \rightarrow \mathbb{C P}^{2}$ и $f^{\prime \prime}: X^{\prime \prime} \rightarrow \mathbb{C P}^{2}$ степени $N$, разветвленных соответственно вдоль каспидальных кривых Гурвица $\bar{H}^{\prime}$ и $\bar{H}^{\prime \prime}$ 
(возможно, с отрицательными ноудами). Пусть $s^{\prime}=\operatorname{bmf}\left(\bar{H}^{\prime}\right)$ и $s^{\prime \prime}=\operatorname{bmf}\left(\bar{H}^{\prime \prime}\right)-$ разложения на множители брэйд-монодромии кривых $\bar{H}^{\prime}$ и $\bar{H}^{\prime \prime}$, а накрытие $f^{\prime}$ (соответственно $f^{\prime \prime}$ ) определено монодромией $\mu^{\prime}: G\left(s^{\prime}\right) \rightarrow \mathscr{S}_{N}$ (соответственно $\left.\mu^{\prime \prime}\right)$. Скажем, что $\bar{H}^{\prime}$ и $\bar{H}^{\prime \prime}$ являются слабо $\mu$-эквивалентными, если пары $\left(s^{\prime}, \mu_{s^{\prime}}\right)$ и $\left(s^{\prime \prime}, \mu_{s^{\prime \prime}}\right)$ слабо $\mu$-эквивалентны. Отметим, что условие слабой $\mu$-эквивалентности означает, что кривые $\bar{H}^{\prime}$ и $\bar{H}^{\prime \prime}$ являются регулярно гомотопными и при этой регулярной гомотопии на каждом шаге сохраняется эпиморфизм из фундаментальных групп дополнений к соответствующим кривым в симметрическую группу.

ПреДЛОЖеНИЕ 9.1. Пусть две кривые Гурвица $\bar{H}^{\prime}$ и $\bar{H}^{\prime \prime}$ являются кривыми ветвления двух общих накрытии $f^{\prime}: X^{\prime} \rightarrow \mathbb{C P}^{2}$ u $f^{\prime \prime}: X^{\prime \prime} \rightarrow \mathbb{C P}^{2}, u$ пусть $\omega^{\prime}$ и $\omega^{\prime \prime}$ - симплектические формы на $X^{\prime}$ и $X^{\prime \prime}$ соответственно, построенные с помощью конструкиии, использованной в доказательстве теоремы 8.1. Если $\bar{H}^{\prime}$ и $\bar{H}^{\prime \prime}$ являются слабо н-эквивалентными, то существует симплектоморфизм $h:\left(X^{\prime}, \omega^{\prime}\right) \rightarrow\left(X^{\prime \prime}, \omega^{\prime \prime}\right)$ такой, что накрытия $f^{\prime} u f^{\prime \prime} \circ h$ являются изотопными.

ДоКАЗАТЕЛЬСТво следует из определения слабой $\mu$-эквивалентности и по существу повторяет доказательство теоремы 9.2.

Пусть $(X, \omega)$ - компактное симплектическое четырехмерное многообразие с симплектической формой $\omega$, класс когомологий которой $[\omega] \in H^{2}(X, \mathbb{Z})$. Как отмечалось выше, зафиксировав совместимую с $\omega$ почти комплексную структуру $J$ и соответствующую риманову метрику $g$ и рассмотрев линейное расслоение $L$ на $X$, первый класс Черна которого есть [ $\omega]$, Д. Ору в [3] доказал, что для $k \gg 0$ линейное расслоение $L^{\otimes k}$ имеет много почти голоморфных сечений, так что можно выбрать три из них, которые дают почти голоморфное общее накрытие $f_{k}: X \rightarrow \mathbb{C P}^{2}$ степени $N_{k}=k^{2}[\omega]^{2}$, разветвленное вдоль каспидальной кривой Гурвица $\bar{H}$ (возможно, с отрицательными ноудами). Обозначим, как и выше, через $\operatorname{bmf}\left(\bar{H}_{k}\right)=s_{k}$ разложение на множители брэйд-монодромии кривой Гурвица $\bar{H}_{k}$ и через $\mu_{k}: G\left(s_{k}\right) \rightarrow \mathscr{S}_{N_{k}}$ монодромию накрытия $f_{k}$. Пара $\bar{\beta}_{\mu_{k}}(X, \omega)=\left(\operatorname{bmf}\left(\bar{H}_{k}\right), \mu_{k}\right)$ называется $k$-м брэйд-монодромным инвариантом симплектического четырехмерного многообразия $(X, \omega)$.

ТеОРема 9.3 [62]. Если два симплектических четырехмерных многообразия $\left(X^{\prime}, \omega^{\prime}\right)$ u $\left(X^{\prime \prime}, \omega^{\prime \prime}\right)$ симплектоморфны, то для достаточно большого $k$ брэйд-монодромные инварианты $\bar{\beta}_{\mu_{k}}\left(X^{\prime}, \omega^{\prime}\right)$ u $\bar{\beta}_{\mu_{k}}\left(X^{\prime \prime}, \omega^{\prime \prime}\right)$ являются слабо н-эквивалентными.

Кроме того, в [63] получена формула, позволяющая вычислять разложение брэйд-монодромии на множители дискриминантной кривой $\bar{H}_{2 k}$ при $k \gg 0$, если известно $\operatorname{bmf}\left(\bar{H}_{k}\right)$. Однако эта формула слишком громоздка, чтобы приводить ее здесь.

Появление условия слабой эквивалентности в теореме 9.3 связано с конструкцией изотопии накрытий, которая непрерывно зависит от выбора ручных почти комплексных структур. Поэтому для симплектических четырехмерных многообразий мы получаем регулярную гомотопию кривых ветвления, которая приводит к слабой $\mu$-эквивалентности их брэйд-монодромных разложений 
на множители. Следующая теорема показывает, что в общем случае мы не можем избежать рождения и уничтожения пар положительных и отрицательных ноудов.

ТЕОРема 9.4 [24]. Для любого $N \geqslant 4$ существуют два изотопнъх общих накрытия $f_{1}: X \rightarrow \mathbb{C P}^{2}$ и $f_{2}: X \rightarrow \mathbb{C P}^{2}$ степени $N$, разветвленные вдоль каспидальных кривых Гурвица $\bar{H}_{1}$ и $\bar{H}_{2}$ без отрицательных ноудов, такие, что

(i) $\bar{H}_{1}$ и $\bar{H}_{2}$ имеют разные типъ брэйд-монодромных разложений на множители;

(ii) пары $\left(\operatorname{bmf}\left(\bar{H}_{1}\right), \mu_{1}\right) u\left(\operatorname{bmf}\left(\bar{H}_{2}\right), \mu_{2}\right)$ слабо $\mu$-эквивалентны.

Доказательство этой теоремы состоит в следующем. Пусть $\bar{H}_{N}-$ дискриминантная кривая общей линейной проекции pr: $X_{N} \rightarrow \mathbb{C P}^{2}$ гладкой проективной поверхности $X_{N} \subset \mathbb{C P}^{3}$ степени $\operatorname{deg} X_{N}=N$. Обозначим через $m=N(N-1)$ степень кривой $\bar{H}_{N}$ и через $s=s_{N}=\operatorname{bmf}\left(\bar{H}_{N}\right)$ разложение на множители ее брэйд-монодромии. Проекция pr определяет общий эпиморфизм (монодромию) $\mu=\mu_{N}: G(s) \rightarrow \mathscr{S}_{N}$. B [6] доказано, что $C$-группа $G(s) C$-изоморфна группе кос $\operatorname{Br}_{N}$ и $\mu$ совпадает с каноническим эпиморфизмом $\gamma_{N}: \operatorname{Br}_{N} \rightarrow \mathscr{S}_{N}$, имеющим своим ядром группу крашеных кос. Применяя несколько раз формулу разложения на множители полного поворота с удвоенным числом нитей, строим две пары $\left(\bar{s}, \mu_{\bar{s}}\right)$ и $\left(\tilde{s}, \mu_{\tilde{s}}\right), \bar{s}, \tilde{s} \in S_{A_{0} \cup A_{1} \cup A_{2}} \subset S_{\mathrm{Br}_{M}}$ для некоторого $M$, такие, что:

1) $\alpha(\bar{s})=\alpha(\tilde{s})=\Delta_{M}^{2}$;

2) $G_{\bar{s}}$ является $C$-изоморфной $C$-группе $G(s) \simeq \operatorname{Br}_{N}$, а $G_{\tilde{s}}$ является $C$-изоморфной $C$-группе $\mathrm{Br}_{N} /\left\{\left[a_{1}, a_{2}^{-2} a_{3} a_{2}^{2}\right]=\mathbf{1}\right\}$, и, следовательно, элементы $\bar{s}$ и $\tilde{s}$ имеют различные типы разложений на множители;

3) $\mu_{\bar{s}}$ и $\mu_{\tilde{s}}$ являются общими гомоморфизмами в группу $\mathscr{S}_{N}$;

4) пары $\left(\bar{s}, \mu_{\bar{s}}\right)$ и $\left(\tilde{s}, \mu_{\tilde{s}}\right)$ слабо $\mu$-эквивалентны.

Согласно теореме 4.2 существуют две каспидальные кривые Гурвица $\bar{H}_{1}$ и $\bar{H}_{2}$ в $\mathbb{C P}^{2}$ степени $M$ такие, что $\operatorname{bmf}\left(\bar{H}_{1}\right)=\bar{s}$ и $\operatorname{bmf}\left(\bar{H}_{2}\right)=\tilde{s}$. Общие гомоморфизмы $\mu_{\bar{s}}$ и $\mu_{\tilde{s}}$ определяют два общих накрытия $f^{\prime}: X^{\prime} \rightarrow \mathbb{C P}^{2}$ и $f^{\prime \prime}$ : $X^{\prime \prime} \rightarrow \mathbb{C P}^{2}$, разветвленные вдоль $\bar{H}_{1}$ и $\bar{H}_{2}$ соответственно. Из предложения 9.1 следует, что существует симплектоморфизм $h: X^{\prime} \rightarrow X^{\prime \prime}$ такой, что накрытия $f_{1}=f^{\prime}: X=X^{\prime} \rightarrow \mathbb{C P}^{2}$ и $f_{2}=f^{\prime \prime} \circ h: X \rightarrow \mathbb{C P}^{2}$ являются изотопными, но их гурвицевские кривые ветвления имеют разные типы разложений на множители брэйд-монодромии.

\section{§ 10. Циклические накрытия проективной плоскости}

Циклические накрытия являются простейшими, наиболее хорошо изученными примерами накрытий проективной плоскости.

10.1. Вложение циклических накрытий плоскости в проективные комплексные трехмерные рациональные многообразия. Пусть, как и выше, $\bar{H} \subset \mathbb{C P}^{2}$ - кривая Гурвица степени $m$ (без отрицательных ноудов). Зафиксируем "бесконечно удаленную" прямую $R_{\infty}$. Согласно лемме 5.1 мы можем считать (заменив на $H$-изотопную кривую), что $\bar{H}$ является множеством нулей гладкого сечения $\bar{s}_{m}$ линейного расслоения $p: \mathscr{L}(m) \rightarrow \mathbb{C P}^{2}$, ассоциированного 
с пучком $\mathscr{O}_{\mathbb{C P}^{2}}(m)$ (см. п. 5.4), являющегося комплексно-аналитическим в некоторой окрестности прямой $R_{\infty}$ и в некоторой окрестности всех критических точек кривой $\bar{H}$. Кроме того, сделав перемасштабирование, можем считать, что $\bar{H}$ является симплектической поверхностью.

$C$-гомоморфизм $\nu: \pi_{1} \rightarrow \mathbb{F}_{1}$, где $\pi_{1}=\pi_{1}\left(\mathbb{C}^{2} \backslash H\right)$ - фундаментальная группа дополнения к аффинной кривой Гурвица, определяет бесконечное циклическое неразветвленное накрытие $f=f_{\infty}: X_{\infty}^{\prime} \rightarrow X^{\prime}=\mathbb{C}^{2} \backslash H$. Имеем $H_{1}\left(X_{\infty}^{\prime}, \mathbb{Z}\right)=$ $N / N^{\prime}$, где $N=\operatorname{ker} \nu$, и действие $h$ на $H_{1}\left(X_{\infty}^{\prime}, \mathbb{Z}\right)$ совпадает с действием порождающего элемента группы накрывающих преобразований накрытия $f_{\infty}$. Для произвольного $n \in \mathbb{N}$ обозначим через $\bmod _{n}: \mathbb{F}_{1} \rightarrow \mu_{n}=\mathbb{F}_{1} /\left\{h^{n}\right\}$ естественный эпиморфизм на циклическую группу $\mu_{n}$ порядка $n$. Накрытие $f_{\infty}$ может быть пропущено через циклическое накрытие $f_{n}: X_{n}^{\prime} \rightarrow \mathbb{C}^{2} \backslash H$, ассоциированное с эпиморфизмом $\bmod _{n} \circ \nu, f_{\infty}=g_{n} \circ f_{n}$.

Для того чтобы продолжить накрытие $f_{n}: X_{n}^{\prime} \rightarrow \mathbb{C}^{2} \backslash H$ до некоторого конечного накрытия над всем $\mathbb{C P}^{2}$, обозначим через $d$ наименьшее неотрицательное целое число такое, что $m+d$ делится нацело на $n$. Положим $m+d=k n$ и рассмотрим линейное расслоение $p: \mathscr{L}(k) \rightarrow \mathbb{C P}^{2}$, ассоциированное с пучком $\mathscr{O}_{\mathbb{C P}^{2}}(k)$. Обозначим через $\bar{s}_{d}$ сечение расслоения $\mathscr{L}(d)$, заданного (в обозначениях из п. 5.4) над $\mathbb{C}_{2}^{2}$ функцией $w_{2}=u_{2}^{d}$. Сечение $\bar{s}_{d}$ обращается в нуль вдоль прямой $R_{\infty}$ с кратностью $d$.

Произведение $\bar{s}_{m+d}=\bar{s}_{m} \bar{s}_{d}$ является сечением расслоения $\mathscr{L}(m+d)$, где $\bar{s}_{m}-$ это сечение расслоения $\mathscr{L}(m)$, нулями которого является кривая $\bar{H}$.

Определим подмногообразие (с аналитическими особенностями) $\tilde{X}_{n} \hookrightarrow \mathscr{L}(k)$ с помощью уравнений

$$
w_{i}^{n}=\bar{s}_{m+d}\left(u_{i}, v_{i}\right)
$$

в картах $W_{i} \subset \mathscr{L}(k)$ и положим $\tilde{f}_{n}=p_{\mid X_{n}}$, где $p: \mathscr{L}(k) \rightarrow \mathbb{C P}^{2}-$ морфизм, задающий на $\mathscr{L}(k)$ структуру линейного расслоения. Имеем $\tilde{f}_{n}^{-1}\left(\mathbb{C}^{2} \backslash H\right) \simeq X_{n}^{\prime}$ и ограничение отображения $\tilde{f}_{n}$ на $X_{n}^{\prime}$ совпадает с $f_{n}^{\prime}$. Поэтому $\tilde{f}_{n}: \widetilde{X}_{n} \rightarrow \mathbb{C P}^{2}$ является продолжением отображения $f_{n}^{\prime}: X_{n}^{\prime} \rightarrow X^{\prime}$ на многообразие $\widetilde{X}_{n}$. Сделав перемасштабирование $w_{i} \mapsto \varepsilon w_{i}$, можем считать, что многообразие $\widetilde{X}_{n}$ вложено в проективное комплексное рациональное трехмерное многообразие $\overline{\mathscr{L}}(k)$ (некоторую гладкую проективную компактификацию многообразия $\mathscr{L}(k))$ как симплектическое подмногообразие с аналитическими особенностями. Согласно следствию 9.1 мы можем разрешить особые точки многообразия $\widetilde{X}_{n}$ с помощью композиции моноидальных преобразований $\sigma$ с неособыми центрами и получить неособое симплектическое многообразие $\bar{X}_{n}$, вложенное как симплектическое подмногообразие в рациональное проективное трехмерное комплексное многообразие, симплектическая структура на котором задана некоторой целочисленной кэлеровой формой.

Отображение $\bar{f}_{n}=\tilde{f}_{n} \circ \sigma: \bar{X}_{n} \rightarrow \mathbb{C P}^{2}$ не является конечным разветвленным накрытием только в точках объединения $E$ множеств $\bar{f}_{n}^{-1}\left(p_{i}\right)=E_{i}$ прообразов особых точек $p_{i} \in \operatorname{Sing} \bar{H}$ и в точках множества $\bar{f}_{n}^{-1}\left(\bar{H} \cap R_{\infty}\right)=E_{\infty}$, если $d \neq 0$. Множество $E_{i}$ совпадает с прообразом поверхностной особой точки, заданной уравнением $w^{n}=F(u, v)$, при разрешении этой особой точки, где $F(u, v)=0$ - локальное уравнение кривой $\bar{H}$ в точке $p_{i}$. Прообраз $\bar{f}_{n}^{-1}\left(R_{\infty}\right)$ распадается на две части $E_{\infty}$ и $R_{\infty}^{\prime}$, и легко видеть, что ограничение $\bar{f}_{n}$ на $R_{\infty}^{\prime}$ 
является $n_{0}$-листным циклическим накрытием, разветвленным над точками из $\bar{H} \cap R_{\infty}$, где $n_{0}=\operatorname{HОД}(n, d)$ (если $d=0$, то $n_{0}=n$ ), и индекс ветвления отображения $\bar{f}_{n}$ вдоль $R_{\infty}^{\prime}$ равен $n_{\infty}=n / n_{0}$. Над каждой точкой $q_{j} \in \bar{H} \cap R_{\infty}$ множество $E_{\infty, j}$ совпадает с прообразом поверхностной особой точки, заданной уравнением $w^{n}=u v^{n_{\infty}}$, при разрешении этой особой точки.

Из приведенного выше описания разрешения особых точек следует, что если мы знаем числа $m$ и $n$, а также знаем локальные уравнения всех особых точек кривой $\bar{H}$, то, пользуясь аддитивностью эйлеровой характеристики, мы можем вычислить эйлерову характеристику $e$ многообразия $\bar{X}_{n}$. Поэтому для вычисления чисел Бетти $b_{i}=\operatorname{dim} H_{i}\left(\bar{X}_{n}, \mathbb{R}\right)$ многообразия $\bar{X}_{n}$ достаточно уметь вычислять только первое число Бетти $b_{1}$, так как $e=\sum_{i=0}^{4}(-1)^{i} b_{i}$, а по двойственности Пуанкаре $b_{1}=b_{3}$ и $b_{0}=b_{4}=1$.

Пространство $H_{1}\left(\bar{X}_{n}, \mathbb{Q}\right)$ может быть описано в терминах модуля Александера кривой $\bar{H}$, а первое число Бетти может быть выражено через число корней многочлена Александера этой кривой.

\section{2. Модули Александера и многочлены Александера кривых} Гурвица. По определению модуль Александера $A_{0}(\bar{H})$ и многочлен Александера $\Delta(t)$ кривой Гурвица $\bar{H}$ - это модуль Александера $A_{0}\left(\pi_{1}\right)$ и многочлен Александера $\Delta(t)$ фундаментальной группы $\pi_{1}=\pi_{1}\left(\mathbb{C}^{2} \backslash H\right)$ дополнения к аффинной кривой Гурвица $H$.

Как было отмечено в $\S 7$, множество фундаментальных групп $\pi_{1}=\pi_{1}\left(\mathbb{C}^{2} \backslash H\right)$ совпадает с множеством гурвицевских $C$-групп.

Свойства модулей и многочленов Александера гурвицевских $C$-групп были описаны в пп. 3.7-3.10. Применяя их к кривым Гурвица, получаем следующие утверждения.

ТеОрема 10.1. Пусть $\bar{H}$ - кривая Гурвица степени $m$ и $A_{0}(\bar{H})$ - ее модуль Александера. Тогда

(i) $\Lambda$-модуль $A_{0}(\bar{H})$ является конечно порожденным $\mathbb{Z}$-модулем;

(ii) модуль $A_{0}(\bar{H})$ является фактормодулем $\Lambda$-модуля $\mathbb{Z} \oplus\left(\Lambda /\left(t^{m}-1\right) \Lambda\right)^{m-2}$, где $\mathbb{Z}$ - тривиальный $\Lambda$-модуль;

(iii) действие $t$ на $A_{0}(\bar{H}) \otimes \mathbb{C}$ является полупростым;

(iv) если $\bar{H}$ - неприводимая кривая Гурвица, то $A_{0}(\bar{H})$ является нётеровым $(t-1)$-обратимым $t$-унипотентным $\Lambda$-модулем, верно и обратное утверждение;

(v) если $\bar{H}$ - неприводимая кривая Гурвица степени $\operatorname{deg} \bar{H}=p^{n}$, где $p-$ простое число, то группа $\pi_{1}^{\prime} / \pi_{1}^{\prime \prime}$ является конечной, где $\pi_{1}=\pi_{1}\left(\mathbb{C}^{2} \backslash H\right)$.

СлеДСтвиЕ 10.1. Пусть $\bar{H}$ - кривая Гурвица степени $m u \Delta(t)$ - ее многочлен Александера. Тогда

(i) $\Delta(t) \in \mathbb{Z}[t]$;

(ii) $\Delta(0)= \pm 1$;

(iii) многочлен $\Delta(t)$ является делителем многочлена $(t-1)\left(t^{m}-1\right)^{m-2} u$, следовательно, корни многочлена $\Delta(t)$ являются корнями степени $m$ из единиць;

(iv) если $\bar{H}$ состоит из $k$ неприводимых компонент, то кратность корня $t=1$ ее многочлена Александера $\Delta(t)$ равна $k-1$. 
СлеДСтвиЕ 10.2. Если $\bar{H}$ - неприводимая кривая Гурвица, то

(i) $\Delta(t)$ является возвратным многочленом, т.е. имеет место равенство $\Delta(t)=t^{\operatorname{deg} \Delta(t)} \Delta\left(t^{-1}\right)$

(ii) $\operatorname{deg} \Delta(t)$ является четным числом;

(iii) $\Delta(1)=1$;

(iv) если $\operatorname{deg} \bar{H}=p^{n}$, где $p$ - простое число, то $\Delta(t) \equiv 1$.

$\Lambda$-модуль $A_{n}(\bar{H})=A_{0}(\bar{H}) /\left(t^{n}-1\right) A_{0}(\bar{H})$ называется $n$-м производным модулем Александера кривой $\bar{H}$. Из теоремы 10.1 следует, что для кривой Гурвица, состоящей из $k$ неприводимых компонент, $\mathbb{Q}\left[t, t^{-1}\right]$-модуль $A_{0}(\bar{H}) \otimes \mathbb{Q}$ раскладывается в прямую сумму: $A_{0}(\bar{H}) \otimes \mathbb{Q}=A_{\neq 1}(\bar{H}) \oplus A_{1}$, где $A_{1} \simeq \mathbb{Q}^{k-1}$ - это ядро умножения на $t-1$. Обозначим через $A_{n, \neq 1}(\bar{H})$ образ подмодуля $A_{\neq 1}$ при естественном гомоморфизме $A_{0}(\bar{H}) \otimes \mathbb{Q} \rightarrow A_{n}(\bar{H}) \otimes \mathbb{Q}$, и пусть $r_{n}$ (соответственно $\left.r_{n, \neq 1}\right)$ - число корней многочлена Александера $\Delta(t)$ кривой $\bar{H}$, являющихся (соответственно не равными 1 ) корнями степени $n$ из единицы.

10.3. Вычисление группы первых гомологий циклического накрытия плоскости. Как отмечалось выше, пространство $H_{1}\left(\bar{X}_{n}, \mathbb{Q}\right)$ может быть описано в терминах модуля Александера кривой $\bar{H}$, а первое число Бетти может быть выражено через число корней многочлена Александера этой кривой.

Теорема 10.2 [15]. Пусть $\bar{X}_{n}$ - разрешение особенностей $n$-листного ииклического накрытия, разветвленного вдоль кривой Гурвица $\bar{H}$ и, возможно, вдоль $R_{\infty}$. Тогда

(i) $H_{1}\left(\bar{X}_{n}, \mathbb{Q}\right) \simeq A_{n, \neq 1}(\bar{H})$;

(ii) $b_{1}\left(\bar{X}_{n}\right)=r_{n, \neq 1}$.

Приведем набросок доказательства этой теоремы. Рассмотрим снова циклическое накрытие $f=f_{\infty}: X_{\infty} \rightarrow X^{\prime}=\mathbb{C}^{2} \backslash H$, соответствующее каноническому эпиморфизму $\nu: \pi_{1}\left(\mathbb{C}^{2} \backslash H\right) \rightarrow \mathbb{F}_{1}$. Пространство $X^{\prime}$ будем рассматривать как факторпространство $X^{\prime}=X_{\infty} / \mathbb{F}_{1}$. В этой ситуации Дж. Милнор [64] рассмотрел точную последовательность цепных комплексов

$$
0 \rightarrow C_{\bullet}\left(X_{\infty}\right) \stackrel{h-\mathrm{id}}{\longrightarrow} C_{\bullet}\left(X_{\infty}\right) \stackrel{\varphi_{*}}{\longrightarrow} C_{\bullet}\left(X^{\prime}\right) \rightarrow 0,
$$

которая приводит к точной последовательности групп гомологий

$$
\cdots \rightarrow H_{1}\left(X_{\infty}\right) \stackrel{h-\mathrm{id}}{\longrightarrow} H_{1}\left(X_{\infty}\right) \stackrel{f_{*}}{\longrightarrow} H_{1}\left(X^{\prime}\right) \rightarrow H_{0}\left(X_{\infty}\right) \rightarrow 0
$$

(будем писать $h$ вместо $h_{*}$, если это не приводит к недоразумению).

Действие $h$ определяет на каждой группе $H_{i}\left(X_{\infty}\right)$ структуру $\Lambda$-модуля (так что $H_{1}\left(X_{\infty}\right) \simeq N / N^{\prime}$ - это модуль Александера $C$-группы $\left.G=\pi_{1}\right)$. Если группы $H_{i}\left(X^{\prime}\right)$ рассматривать как $\Lambda$-модули, на которых $t$ действует тривиально, то последовательность (24) превращается в точную последовательность $\Lambda$-модулей. Отметим, что действие $t \in \Lambda$ на группе $H_{0}\left(X_{\infty}\right) \simeq \mathbb{Z}$ является тривиальным, т. е. умножение на $t$ является тождественным автоморфизмом группы $H_{0}\left(X_{\infty}\right)$.

Если $G_{n} \subset \mathbb{F}_{1}$ - бесконечная циклическая группа, порожденная элементом $h^{n}$, то $X_{n}^{\prime}=X_{\infty} / G_{n}$ и $X^{\prime}=X_{n}^{\prime} / \mu_{n}$, где $\mu_{n}=\mathbb{F}_{1} / G_{n}$ - циклическая группа 
порядка $n$. Обозначим через $h_{n}$ автоморфизм пространства $X_{n}^{\prime}$, индуцированный монодромией $h$. Тогда $h_{n}$ является порождающим элементом группы накрывающих преобразований $\operatorname{Deck}\left(X_{n}^{\prime} / X^{\prime}\right)=\mu_{n}$, действующей на $X_{n}^{\prime}$. Применим последовательность

$$
\cdots \rightarrow H_{1}\left(X_{\infty}\right) \stackrel{h^{n}-\mathrm{id}}{\longrightarrow} H_{1}\left(X_{\infty}\right) \stackrel{g_{n, *}}{\longrightarrow} H_{1}\left(X_{n}^{\prime}\right) \rightarrow H_{0}\left(X_{\infty}\right) \rightarrow 0,
$$

построенную аналогично последовательности (24), к бесконечному циклическому накрытию $g_{n}=g_{\infty, n}: X_{\infty} \rightarrow X_{n}^{\prime}$ для исследования группы $H_{1}\left(X_{n}^{\prime}\right)$.

Обозначим $X_{n}=\bar{X}_{n} \backslash\left(E \cup E_{\infty}\right)$. Имеем два вложения $i_{n}: X_{k}^{\prime} \hookrightarrow X_{n}$ и $j_{n}$ : $X_{n} \hookrightarrow \bar{X}_{n}$.

Действие $h_{n}$ на многообразии $X_{n}$ индуцирует на группе $H_{1}\left(X_{n}, \mathbb{Z}\right)$ (соответственно на $\left.H_{1}\left(X_{n}^{\prime}, \mathbb{Z}\right)\right)$ структуру $\Lambda$-модуля такую, что гомоморфизм

$$
i_{k *}: H_{1}\left(X_{n}^{\prime}, \mathbb{Z}\right) \rightarrow H_{1}\left(X_{n}, \mathbb{Z}\right),
$$

индуцированный вложением $i_{n}: X_{n}^{\prime} \hookrightarrow X_{n}$, является $\Lambda$-гомоморфизмом. Очевидно, что гомоморфизм $i_{n *}$ является эпиморфизмом.

Обозначим $H_{1}\left(X_{\infty}, \mathbb{Q}\right)_{n}=\left\{v \in H_{1}\left(X_{\infty}, \mathbb{Q}\right) \mid h_{*}^{n}(v)=v\right\} . \quad$ Пространство $H_{1}\left(X_{\infty}, \mathbb{Q}\right)_{n}$ раскладывается в прямую сумму

$$
H_{1}\left(X_{\infty}, \mathbb{Q}\right)_{n}=H_{1}\left(X_{\infty}, \mathbb{Q}\right)_{n, \neq 1} \oplus H_{1}\left(X_{\infty}, \mathbb{Q}\right)_{1}
$$

инвариантных подпространств, где $H_{1}\left(X_{\infty}, \mathbb{Q}\right)_{1}=\left\{v \in H_{1}\left(X_{\infty}, \mathbb{Q}\right) \mid h_{*}(v)=v\right\}$. Очевидно, что $H_{1}\left(X_{\infty}, \mathbb{Q}\right)_{n, \neq 1} \simeq A_{n, \neq 1}(\bar{H}) \otimes \mathbb{Q}$. Имеем $\operatorname{dim} H_{1}\left(X_{\infty}, \mathbb{Q}\right)_{n}=r_{n}$ и $\operatorname{dim} H_{1}\left(X_{\infty}, \mathbb{Q}\right)_{n, \neq 1}=r_{n, \neq 1}$. Из теоремы 10.1 следует, что

$$
r_{n}-r_{n, \neq 1}=r_{1}=\#\{\text { неприводимые компоненты кривой } \bar{H}\}-1 \text {. }
$$

Последовательность $(25)$ приводит к точной последовательности $\mathbb{Q}\left[t, t^{-1}\right]$-модулей

$$
0 \rightarrow H_{1}\left(X_{\infty}, \mathbb{Q}\right)_{n} \longrightarrow H_{1}\left(X_{n}^{\prime}\right) \rightarrow H_{0}\left(X_{\infty}\right) \rightarrow 0 .
$$

Отсюда получаем, что

(i) $\operatorname{dim} H_{1}\left(X_{n}^{\prime}, \mathbb{Q}\right)=r_{n}+1$,

(ii) $\operatorname{dim} H_{1}\left(X_{n}^{\prime}, \mathbb{Q}\right)_{1}=r_{1}+1=\#\{$ неприводимые компоненты кривой $\bar{H}\}$.

Пусть кривая Гурвица $\bar{H}$ состоит из $k$ неприводимых компонент $\bar{H}_{1}, \ldots, \bar{H}_{k}$. Выберем прямую $R \subset \mathbb{C}^{2}$, принадлежащую пучку прямых (относительно которого кривая $\bar{H}$ определена) и трансверсально пересекающую кривую $H$. Обозначим через $\gamma_{i}$ окружность малого радиуса, лежащую в $R$, с центром в одной из точек пересечения $H_{i} \cap R$. Легко видеть, что циклы $\gamma_{1}, \ldots, \gamma_{k}$, соответствующие выбранным окружностям, образуют базис группы $H_{1}\left(\mathbb{C}^{2} \backslash H, \mathbb{Z}\right)$ и не зависят от выбора прямой $R$. Пусть $\bar{\gamma}_{i}, i=1, \ldots, k,-$ цикл в $H_{1}\left(X_{n}^{\prime}, \mathbb{Z}\right)$, соответствующий простому пути $f_{n}^{-1}\left(\gamma_{i}\right)$. Очевидно, все циклы $\bar{\gamma}_{i}, i=1, \ldots, k$, инвариантны при действии $h_{n}$, и легко показать, что они линейно независимы в $H_{1}\left(X_{n}^{\prime}, \mathbb{Z}\right)$ и образуют базис в $H_{1}\left(X_{n}^{\prime}\right)_{1}$.

Следующие две леммы завершают доказательство теоремы 10.2.

Лемма 10.1. Индуцированный гомоморфизм $i_{1 *}: H_{1}\left(X_{n}^{\prime}\right) \rightarrow H_{1}\left(X_{n}\right)$ является эпиморфизмом, ядро которого $\operatorname{ker} i_{1 *}$ равно $H_{1}\left(X_{n}^{\prime}\right)_{1}$. 
Лемма 10.2. Гомоморфизм $i_{2 *}: H_{1}\left(X_{n}, \mathbb{Q}\right) \rightarrow H_{1}\left(\bar{X}_{n}, \mathbb{Q}\right)$ является изоморфизмом.

Из теорем 10.2, 10.1 и следствий 10.1, 10.2 вытекают следующие утверждения.

СЛЕДСТВИЕ 10.3. Пусть $\bar{X}_{n}$ - разрешение особенностей $n$-листного циклического накрытия, разветвленного вдоль кривой Гурвица $\bar{H}$ и, возможно, вдоль прямой $R_{\infty}$. Если $m=\operatorname{deg} \bar{H}$ и п взаимно просты, то $b_{1}\left(\bar{X}_{n}\right)=0$.

СЛЕДСТВИЕ 10.4. Пусть $\bar{X}_{n}$ - разрешение особенностей $n$-листного циклического накрытия, разветвленного вдоль неприводимой кривой Гурвица $\bar{H}$ $u$, возможно, вдоль $R_{\infty}$. Тогда

(i) $b_{1}\left(\bar{X}_{n}\right)$ является четным числом;

(ii) если $\operatorname{deg} \bar{H}=p^{k}$, где $p$ - простое число, то $H_{1}\left(\bar{X}_{n}, \mathbb{Z}\right)$ является конечной группой для любого $n$;

(iii) группа $H_{1}\left(\bar{X}_{p^{n}}, \mathbb{Z}\right)$ является конечной для любого простого числа $p$ и любого $n$.

Отметим, что в [15] было доказано существование для любого $k \in \mathbb{N}$ кривой Гурвица $\bar{H}_{k}$, состоящей из двух неприводимых компонент, такой, что разрешение особенностей $\bar{X}_{k, 6}$ циклического накрытия степени шесть проективной плоскости, разветвленного вдоль $\bar{H}_{k}$, имеет первое число Бетти $b_{1}\left(\bar{X}_{k, 6}\right)$, равное $k$. Напомним, что $b_{1}\left(\bar{X}_{n}\right)$ всегда является четным числом, если $\bar{H}$ является плоской алгебраической кривой, поэтому эти $\bar{H}_{k}$ не могут быть изотопны алгебраическим кривым, если $k$ нечетно.

\section{Список литературы}

[1] A. Hurwitz, "Ueber Riemann'sche Flachen mit gegebenen Verzweigungspunkten", Math. Ann., 39:1 (1891), 1-60.

[2] K. Stein, "Analytische Zerlegungen komplexer Räume", Math. Ann., 132:1 (1956), 63-93.

[3] D. Auroux, "Symplectic 4-manifolds as branched coverings of $\mathbb{C P}^{2}$ ", Invent. Math., 139:3 (2000), 551-602.

[4] O. Chisini, "Una suggestiva rappresentazione reale per le curve algebriche piane", Ist. Lombardo Rend. II. Ser., 66 (1933), 1141-1155.

[5] O. Chisini, "Courbes de diramation des plans multiples et tresses algébriques", Deuxième Colloque de Géométrie Algébrique (Liège, 1952), Georges Thone, Liège; Masson \& Cie, Paris, 1952, 11-27.

[6] B. Moishezon, "Stable branch curves and braid monodromies", Algebraic geometry (Chicago, IL, 1980), Lecture Notes in Math., 862, Springer, Berlin-New York, 1981, 107-192.

[7] B. Moishezon, "Algebraic surfaces and the arithmetic of braids. I", Arithmetic and geometry, vol. II, Progr. Math., 36, Birkhäuser Boston, Boston, MA, 1983, 199-269.

[8] B. Moishezon, "Algebraic surfaces and the arithmetic of braids. II", Combinatorial methods in topology and algebraic geometry (Rochester, NY, 1982), Contemp. Math., 44, Amer. Math. Soc., Providence, RI, 1985, 311-344.

[9] B. Moishezon, M. Teicher, "Braid group technique in complex geometry. I. Line arrangements in $\mathbb{C} P^{2}$ ", Braids (Santa Cruz, CA, 1986), Contemp. Math., 78, Amer. Math. Soc., Providence, RI, 1988, 425-555. 
[10] B. Moishezon, M. Teicher, "Braid group technique in complex geometry. II. From arrangements of lines and conics to cuspidal curves", Algebraic geometry (Chicago, IL, 1989), Lecture Notes in Math., 1479, Springer, Berlin, 1991, 131-180.

[11] B. Moishezon, M. Teicher, "Braid group techniques in complex geometry. III. Projective degeneration of $V_{3}$ ", Classification of algebraic varieties (L'Aquila, 1992), Contemp. Math., 162, Amer. Math. Soc., Providence, RI, 1994, 313-332.

[12] B. Moishezon, M. Teicher, "Braid group techniques in complex geometry. IV. Braid monodromy of the branch curve $S_{3}$ of $V_{3} \rightarrow \mathbb{C} P^{2}$ and application to $\pi_{1}\left(\mathbb{C} P^{2}-S_{3}, *\right)$ ", Classification of algebraic varieties (L'Aquila, 1992), Contemp. Math., 162, Amer. Math. Soc., Providence, RI, 1994, 333-358.

[13] B. Moishezon, "The arithmetic of braids and a statement of Chisini", Geometric topology (Haifa, 1992), Contemp. Math., 164, Amer. Math. Soc., Providence, RI, 1994, 151-175.

[14] M. Teicher, "Braid groups, algebraic surfaces and fundamental groups of complements of branch curves", Algebraic geometry (Santa Cruz, 1995), Proc. Sympos. Pure Math., 62, Part 1, Amer. Math. Soc., Providence, RI, 1997, 127-150.

[15] Г.-М. Гроель, Вик. С. Куликов, "О симплектических накрытиях проективной плоскости”, Изв. РАН. Сер. матем., 69:4 (2005), 19-58; англ. пер.: G.-M. Greuel, Vik. S. Kulikov, "On symplectic coverings of the projective plane", Izv. Math., 69:4 (2005), 667-701.

[16] M. J. Micallef, B. White, "The structure of branch points in minimal surfaces and in pseudoholomorphic curves", Ann. of Math. (2), 141:1 (1995), 35-85.

[17] J.-C. Sikorav, "Singularities of J-holomorphic curves", Math. Z., 226:3 (1997), 359-373.

[18] S. Ivashkovich, V. Shevchishin, "Structure of the moduli space in a neighborhood of a cusp-curve and meromorphic hulls", Invent. Math., 136:3 (1999), 571-602.

[19] S. Yu. Orevkov, "Markov moves for quasipositive braids", C. R. Acad. Sci. Paris Sér. I Math., 331:7 (2000), 557-562.

[20] Вик. С. Куликов, В. М. Харламов, “О брэйд-монодромных разложениях на множители”, Изв. РАН. Сер. матем., 67:3 (2003), 79-118; англ. пер.: V.M. Kharlamov, Vik. S. Kulikov, "On braid monodromy factorizations", Izv. Math., 67:3 (2003), 499-534.

[21] E. Artin, "Theorie der Zöpfe", Abhandlungen Hamburg, 4 (1925), 47-72.

[22] F. A. Garside, "The braid group and other groups", Quart. J. Math. Oxford Ser. (2), 20 (1969), 235-254.

[23] T. Ben-Itzhak, M. Teicher, "Properties of Hurwitz equivalence in the braid group of order n", J. Algebra, 264:1 (2003), 15-25.

[24] Вик. С. Куликов, "Формула разложения на множители полного поворота с удвоенным числом нитей”, Изв. РАН. Сер. матем., 68:1 (2004), 123-158; англ. пер.: Vik. S. Kulikov, "A factorization formula for the full twist of double the number of strings", Izv. Math., 68:1 (2004), 125-158.

[25] Вик. С. Куликов, "Многочлены Александера плоских алгебраических кривых", Изв. РАН. Сер. матем., 57:1 (1993), 76-101; англ. пер.: Vik. S. Kulikov, "Alexander polynomials of plane algebraic curves", Russian Acad. Sci. Izv. Math., 42:1 (1994), 67-89.

[26] Ю. В. Кузьмин, "Об одном способе построения $C$-групп", Изв. РАН. Сер. матем., 59:4 (1995), 105-124; англ. пер.: Yu. V. Kuz'min, "On a method of constructing C-groups", Izv. Math., 59:4 (1995), 765-783.

[27] О.В. Куликова, "О фундаментальных группах дополнений к кривым Гурвица", Изв. РАН. Сер. матем., 69:1 (2005), 125-132; англ. пер.: О. V. Kulikova, "On the fundamental groups of the complements of Hurwitz curves", Izv. Math., 69:1 (2005), $123-130$. 
[28] Вик. С. Куликов, "Геометрическая реализация С-групп", Изв. РАН. Сер. матем., 58:4 (1994), 194-203; англ. пер.: Vik. S. Kulikov, "A geometric realization of C-groups", Russian Acad. Sci. Izv. Math., 45:1 (1995), 197-206.

[29] J. Stallings, "On fibering certain 3-manifolds", Topology of 3-manifolds and related topics, Proc. The Univ. of Georgia Institute (1961), Prentice-Hall, Englewood Cliffs, NJ, 1962, 95-100.

[30] R. H. Crowell, R. H. Fox, Introduction to knot theory, Based upon lectures given at Haverford College under the Philips Lecture Program, Ginn and Co., Boston, MA, 1963.

[31] Дж. Милнор, Особъе точки комплекснъх гиперповерхностей, Мир, М., 1971; пер. с англ.: J. Milnor, Singular points of complex hypersurfaces, Ann. of Math. Studies, 61, Princeton Univ. Press, Princeton, NJ; University of Tokyo Press, Tokyo, 1968.

[32] Вик. С. Куликов, "Модули Александера неприводимых С-групп”, Изв. РАН. Сер. матем., 72 (2008) (в печати).

[33] Р. Линдон, П. Шупп, Комбинаторная теория групn, Мир, М., 1980; пер. с англ.: R. C. Lyndon, P. E. Schupp, Combinatorial group theory, Ergebnisse der Mathematik und ihrer Grenzgebiete, 89, Springer-Verlag, Berlin-New York, 1977.

[34] Ю. В. Кузьмин, "Группы заузленных компактных поверхностей и центральные расширения", Матем. сб., 187:2 (1996), 81-102; англ. пер.: Yu. V. Kuz'min, "The groups of knotted compact surfaces, and central extensions", Sb. Math., 187:2 (1996), 237-257.

[35] E. Brieskorn, H. Knörrer, Plane algebraic curves, Birkhäuser Verlag, Basel-BostonStuttgart, 1986; пер. с нем.: Ebene algebraische Kurven, Birkhäuser Verlag, BaselBoston-Stuttgart, 1981.

[36] S. Yu. Orevkov, "Realizability of a braid monodromy by an algebraic function in a disk", C. R. Acad. Sci. Paris Sér. I Math., 326:7 (1998), 867-871.

[37] M. Gromov, "Pseudo holomorphic curves in symplectic manifolds", Invent. Math., 82:2 (1985), 307-347.

[38] A. Libgober, "On the homotopy type of the complement to plane algebraic curves", J. Reine Angew. Math., 367 (1986), 103-114.

[39] Вик. С. Куликов, М. Тайхер, "Брэйд-монодромные разложения и диффеоморфные типы", Изв. РАН. Сер. матем., 64:2 (2000), 89-120; англ. пер.: Vik. S. Kulikov, M. Teicher, "Braid monodromy factorizations and diffeomorphism types", Izv. Math., 64:2 (2000), 311-341.

[40] V. Kharlamov, Vik. S. Kulikov, "Diffeomorphisms, isotopies, and braid monodromy factorizations of plane cuspidal curves", C. R. Acad. Sci. Paris Sér. I Math., 333:9 (2001), 855-859.

[41] J.-C. Sikorav, "Dual elliptic planes", Actes des Journées Mathématiques à la Mémoire de Jean Leray, Sémin. Congr., 9, Soc. Math. France, Paris, 2004, 185-207.

[42] D. Auroux, S. K. Donaldson, L. Katzarkov, "Luttinger surgery along Lagrangian tori and non-isotopy for singular symplectic plane curves", Math. Ann., 326:1 (2003), 185-203.

[43] F. Hirzebruch, "Singularities of algebraic surfaces and characteristic numbers", The Lefschetz centennial conference, Part I (Mexico City, 1984), Contemp. Math., 58, Amer. Math. Soc., Providence, RI, 1986, 141-155.

[44] J. Harris, "On the Severi problem", Invent. Math., 84:3 (1986), 445-461.

[45] I. Tyomkin, On Severi varieties on Hirzebruch surfaces, arXiv: math/0607807, 2006.

[46] Вик. С. Куликов, Д. Ору, В. Шевчишин, "Регулярная гомотопия кривых Гурвица", Изв. РАН. Сер. матем., 68:3 (2004), 91-114; англ. пер.: D. Auroux, Vik. S. Kulikov, V. Shevchishin, "Regular homotopy of Hurwitz curves", Izv. Math., 68:3 (2004), 521-542. 
[47] B. Siebert, G. Tian, "On the holomorphicity of genus two Lefschetz fibrations", Ann. of Math. (2), 161:2 (2005), 959-1020.

[48] J.-F. Barraud, "Nodal symplectic spheres in $\mathbb{C P}^{2}$ with positive self-intersection", Internat. Math. Res. Notices, 1999:9, 495-508.

[49] B. Moishezon, M. Teicher, "Simply-connected algebraic surfaces of positive index", Invent. Math., 89:3 (1987), 601-643.

[50] O. Zariski, Algebraic surfaces, Ergebnisse der Mathematik und ihrer Grenzgebiete, 61, Springer-Verlag, New York-Heidelberg, 1971.

[51] G. Baumslag, D. Solitar, "Some two-generator one-relator non-Hopfian groups", Bull. Amer. Math. Soc., 68 (1962), 199-201.

[52] Вик. С. Куликов, "О гипотезе Кизини”, Изв. РАН. Сер. матем., 63:6 (1999), 83-116; англ. пер.: Vik.S. Kulikov, "On Chisini's conjecture", Izv. Math., 63:6 (1999), 1139-1170.

[53] O. Chisini, "Sulla identita birazionale delle funzioni algebriche di due variabili dotate di una medesima curva di diramazione", Ist. Lombardo Sci. Lett. Cl. Sci. Mat. Nat. Rend. (3), 8(77) (1944), 339-356.

[54] С. Ю. Немировский, "К теореме Куликова о гипотезе Кизини", Изв. РАН. Сер. матем., 65:1 (2001), 77-80; англ. пер.: S. Yu. Nemirovski, "Kulikov's theorem on the Chisini conjecture", Izv. Math., 65:1 (2001), 71-74.

[55] F. Catanese, "On a Problem of Chisini", Duke Math. J., 53:1 (1986), 33-42.

[56] Вик. С. Куликов, "О гипотезе Кизини. II", Изв. РАН. Сер. матем., 72 (2008) (в печати).

[57] J. M. Wahl, "Deformations of plane curves with nodes and cusps", Amer. J. Math., 96:4 (1974), 529-577.

[58] D. Gieseker, "Global moduli for surfaces of general type", Invent. Math., 43:3 (1977), 233-282.

[59] Y.-T. Siu, "The complex-analyticity of harmonic maps and the strong rigidity of compact Kähler manifolds", Ann. Math., 112:1 (1980), 73-111.

[60] Вик. С. Куликов, В.М. Харламов, "О вещественных структурах на жестких поверхностях", Изв. РАН. Сер. матем., 66:1 (2002), 133-152; англ. пер.: Vik. S. Kulikov, V. M. Kharlamov, "On real structures on rigid surfaces", Izv. Math., 66:1 (2002), 133-150.

[61] В. С. Куликов, Вик. С. Куликов, "Общие накрытия плоскости с $A$ - $D$ - $E$-особенностями", Изв. РАН. Сер. матем., 64:6 (2000), 65-106; англ. пер.: V.S. Kulikov, Vik. S. Kulikov, "Generic coverings of the plane with $A-D$-E-singularities", Izv. Math., 64:6 (2000), 1153-1195.

[62] D. Auroux, L. Katzarkov, "Branched coverings of $\mathbb{C P}^{2}$ and invariants of symplectic 4-manifolds", Invent. Math., 142:3 (2000), 631-673.

[63] D. Auroux, L. Katzarkov, A degree doubling formula for braid monodromies and Lefschetz pencils, arXiv: math/0605001, 2006.

[64] J.W. Milnor, "Infinite cyclic coverings", Conference on the Topology of Manifolds (Michigan State Univ., E. Lansing, MI, 1967), Prindle, Weber \& Schmidt, Boston, MA, 1968, 115-133.

Вик. С. Куликов (Vik. S. Kulikov)

Математический институт им. В. А. Стеклова РАН

E-mail: kulikov@mi.ras.ru
Поступила в редакцию 16.01.2007 